Acta Crystallographica Section B

Structural Science, Crystal Engineering and Materials

ISSN 2052-5206

\section{T. Janssen* and A. Janner}

Theoretical Physics, University of Nijmegen, Nijmegen, The Netherlands

Correspondence e-mail: t.janssen@science.ru.nl

\title{
Aperiodic crystals and superspace concepts
}

For several decades the lattice periodicity of crystals, as shown by Laue, was considered to be their essential property. In the early sixties of the last century compounds were found which for many reasons should be called crystals, but were not lattice periodic. This opened the field of aperiodic crystals. An overview of this development is given. Many materials of this kind were found, sometimes with very interesting properties. In the beginning the development was slow, but the number of structures of this type increased enormously. In the meantime hundreds of scientists have contributed to this field using a multi-disciplinary approach.

\section{Introduction}

The idea that crystals are built in a periodic fashion put forward by Haüy was confirmed by Laue in 1912. This time the internal microscopic structure was revealed. Before crystals were mainly studied macroscopically by their morphology. The flat surfaces obeyed the Law of Rational Indices and reflected the macroscopic symmetry of the crystal. The symmetry groups belonged to one of 32 crystal classes. In the 19 th century theoreticians also studied the possible microscopic structure and derived 230 types of space groups. Then in 1912 it was shown that the crystal structure can indeed be characterized by one of the 230 space groups. The definition of a crystal adopted this point of view: a crystal has lattice periodicity and belongs to one of the 230 space-group types. For more than 50 years this point of view prevailed, although some anomalies were found in diffraction or morphology. In the early 1960s more materials were found that could be described as a lattice periodic structure with some disturbance affecting this lattice periodicity. The first to give a crystallographic interpretation was de Wolff. We present here the development of the field he initiated. Corresponding to the character of these papers in Acta Crystallographica, this presents a personal view. Others would perhaps put the emphasis somewhere else and cite other publications.

\section{Macroscopic and microscopic three-dimensional symmetry}

The most striking feature of a crystal is the appearance of flat surfaces. These were explained by Haüy (1784). Moreover, he formulated the Law of Rational Indices. The distances from the intersection points of the face with the unit axes are inversely proportional to a set of integers, later called Miller indices (1839). Hessel (1830) showed that the possible orien-
Received 12 June 2014

Accepted 24 June 2014 
tations of the faces have the point-group symmetry of one of the 32 crystallographic point groups. Symmetry considerations have always been important in crystallography. The same point groups then could be used to characterize the physical properties of crystals. A vector property transforms with a well defined three-dimensional representation of the point group, and a tensor field transforms with the tensor product of this vector representation.

In the 19th century it was shown how to combine translations with the point-group elements to generate space groups. Schoenflies and Fedorov showed that there are 230 different space groups in three dimensions. This was the next step in the symmetry consideration of crystals. This work grew to be of great importance when the Braggs showed, by means of Xrays (discovered in 1895 by Röntgen), that a crystal is indeed lattice periodic and may be characterized by a crystallographic space group.

The following step was taken when considering crystals with magnetic moments. Because a magnetic moment is not invariant under time reversal, the latter may be combined with the elements of a point group or space group. Then magnetic point or space groups, also called Shubnikov groups, could be used for the characterization of magnetic crystals.

\section{Incommensurate modulated crystal phases}

In 1964 the group of Pim de Wolff in Delft found an anomaly in the diffraction pattern of dehydrated sodium carbonate (Brouns et al., 1964). The powder diffraction pattern showed peaks that could not be indexed by three integer indices, so it was proposed to use four. Later on a single crystal was made. The anomalous diffraction peaks in the powder diffraction spectrum corresponded to additional diffraction peaks for a single crystal. These could only be indexed using three irrational indices or four integer indices. Choosing three reciprocal basis vectors for the monoclinic structure, the diffraction peaks could be labelled as

$$
\mathbf{H}=h_{1} \mathbf{a}_{1}^{*}+h_{2} \mathbf{a}_{2}^{*}+h_{3} \mathbf{a}_{3}^{*}+h_{4} \mathbf{a}_{4}^{*}, \text { with } \mathbf{a}_{4}^{*}=\alpha \mathbf{a}_{1}^{*}+\gamma \mathbf{a}_{3}^{*} .
$$

The collection of all $\mathbf{H}$ vectors is called the Fourier module. Spots with $h_{4}=0$ are called main reflections, the others satellites. As $\alpha$ and $\gamma$ depend on temperature, there is no basis $\mathbf{a}_{1}^{*}, \mathbf{a}_{2}^{*}, \mathbf{a}_{3}^{*}$ that allows integer indexing, and therefore they are generally irrational numbers. This means that the phase does not have lattice periodicity; it is an aperiodic compound. Mathematically the structure is quasiperiodic, but because according to the mathematical definition lattice periodic structures are also quasiperiodic another term had to be chosen. The phases were called incommensurate modulated phases and the idea was that these satellites were the consequence of a periodic modulation of the atomic positions, having a period not fitting the periodicity of the underlying lattice.

\subsection{Precursors}

Actually, $\gamma-\mathrm{Na}_{2} \mathrm{CO}_{3}$ was not the first material where a deviation from lattice periodicity was observed. Sometimes, additional spots were seen, called 'Gittergeister' (Dehlinger, 1927; Korekawa, 1967), that could be explained by periodically arranged defects. Expressions for the intensity of the satellites were given in Korekawa (1967). Satellites had been observed in many minerals, e.g. plagioclase. Other systems had spin waves with a wavelength that does not fit the lattice periodicity of the crystal. Also, in $\mathrm{NaNO}_{2}$ additional spots were explained as the consequence of periodically arranged domain walls. Incommensurability as an intrinsic crystallographic property was first proposed by de Wolff and co-workers (see Fig. 1; de Wolff, 1974, 1977), followed by our publication (Janner \& Janssen, 1977).

\subsection{Four-dimensional groups in space-time}

By introducing time reversal in symmetry considerations, one actually acts in four-dimensional space-time. A logical consequence then is to study more general groups acting in this space-time. Four-dimensional groups with a discrete lattice subgroup, and a point group that is a subgroup of the product of the three-dimensional orthogonal group, $\mathrm{O}(3)$ and a group of two elements (time reversal and identity) are actually subgroups in four dimensions. These were called generalized magnetic space-time groups. A full list was published in 1968 (Fast \& Janssen, 1968). These groups may occur as symmetry groups of time-dependent electromagnetic fields. They are a subclass of all four-dimensional space groups (Brown et al., 1978). In the latter, the three-dimensional physical space is not always an invariant space under the point group.

This is not the end of the story. In the frame of special relativity, the physical laws are invariant under the general Poincaré group acting in space-time. Therefore, electromagnetic fields may be invariant under a Poincaré space-time group, where the point-group elements are four-dimensional Lorentz transformations. A simple example in two-dimen-

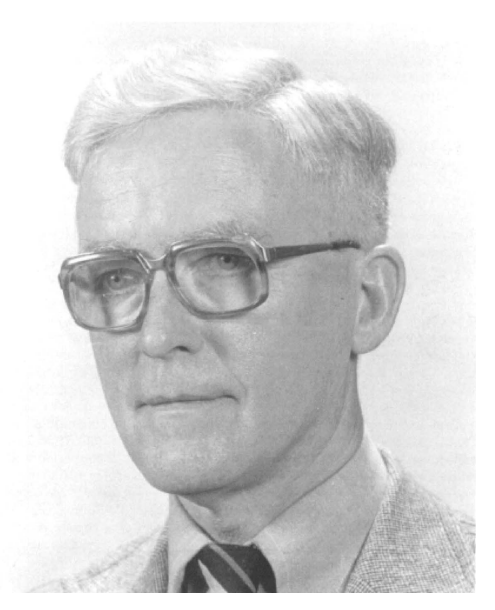

Figure 1

Pim de Wolff (1919-1998), Delft Institute of Technology. 
sional space-time would be a field that is lattice invariant with basis lattice vectors $(1,0)$ and $\left(2,3^{1 / 2}\right)$. This lattice is invariant under a group generated by

$$
A=\left(\begin{array}{cc}
0 & -1 \\
1 & 4
\end{array}\right)
$$

which is equivalent to

$$
\left(\begin{array}{cc}
2+\sqrt{3} & 0 \\
0 & 2-\sqrt{3}
\end{array}\right) \text { and to }\left(\begin{array}{cc}
\cosh (\chi) & \sinh (\chi) \\
\sinh (\chi) & \cosh (\chi)
\end{array}\right)
$$

with $\chi=\operatorname{ArcCosh}[2] \simeq 1.31696$. The first matrix shows the lattice invariance, the second that the transformation gives a dilation along one eigenvector and a contraction along another, and the third shows that it is a Lorentz transformation. Therefore, these elements form a crystallographic Poincaré space-time group. It is not a four-dimensional space group, because the point group is of infinite order. Non-crystallographic Poincaré space-time groups may also be relevant, e.g. a uniform magnetic field and the field of a monochromatic plane wave have as symmetry groups subgroups of the Poincaré group which are Lie groups.

Electromagnetic fields may show point-group or even space-group symmetry. This also holds for electromagnetic potentials. Because the fields are derivatives of the potentials, the symmetry of a field is, generally, not the same as the symmetry of the associated potential. The latter is not unique for the corresponding field. A gauge transformation of the potential leaves the field invariant. If the gauge function is $\chi(\mathbf{r}, t)$, the potentials $\mathbf{A}(\mathbf{r}, t)$ and $V(\mathbf{r}, t)$ transform to $\mathbf{A}+\nabla \chi$ and $V-\partial_{t} \chi$, and these give the same electromagnetic fields. One may then combine Euclidean transformations with gauge transformations such that the potential, and therefore the field as well, is invariant. The gauge transformation is then called a compensating gauge transformation. For example, a constant and homogeneous magnetic field is invariant under an arbitrary translation. The corresponding potential is not invariant under each translation, but the combination of a translation and a compensating gauge transformation leaves the potential invariant.

In quantum mechanics, the gauge transformation acts on the wavefunctions. For a gauge function $\chi$ the wavefunction $\Psi(\mathbf{r})$ transforms to $\exp [i \chi(\mathbf{r})] \Psi(\mathbf{r})$. If $\chi_{g}(\mathbf{r})$ is the compensating gauge transformation for the Euclidean transformation $g$, then the combined action transforms $\Psi(\mathbf{r})$ into $\exp \left[i \chi_{g}(\mathbf{r})\right] \Psi\left(g^{-1} \mathbf{r}\right)$. In the space of electron states, the operator $\left(g, \chi_{g}(\mathbf{r})\right)$ may commute with the Hamiltonian, and is then a symmetry operator for the latter, which has physical consequences. Therefore, compensating gauge transformations are important for physics (Janner \& Janssen, 1971).

\subsection{Higher-dimensional space}

3.3.1. $\gamma-\mathrm{Na}_{2} \mathrm{CO}_{3}$ as four-dimensional periodic structure. Pim de Wolff was the first to propose a crystallographic description of the incommensurate modulated phase $\gamma$ $\mathrm{Na}_{2} \mathrm{CO}_{3}$ (de Wolff, 1974). He considered the phase of the modulation as a free parameter. The positions of the atoms in the unmodulated structure are $\mathbf{n}+\mathbf{r}_{j}$, with $\mathbf{n}$ a lattice vector, and $\mathbf{r}_{j}$ the position of the $j$ th atom in the unit cell. If the positions of the atoms for the modulated structure are given by

$$
\mathbf{r}_{\mathbf{n} j}=\mathbf{n}+\mathbf{r}_{j}+\mathbf{A} \cos \left(\mathbf{q} \cdot \mathbf{n}+\phi_{0}\right),
$$

one may consider lines in four dimensions given by

$$
\left(\mathbf{r}_{\mathbf{n} j}, \phi\right)=\mathbf{n}+\mathbf{r}_{j}+\mathbf{A} \cos (\mathbf{q} \cdot \mathbf{n}+\phi) .
$$

These lines form a pattern with lattice periodicity (Fig. 2). Moreover, the Fourier transform of these lines consists of delta peaks on the lattice points of the four-dimensional reciprocal lattice. It is easy to show that the pattern of equation (1) has lattice periodicity with basis vectors

$$
(\mathbf{a},-\mathbf{q} \cdot \mathbf{a}),(\mathbf{b},-\mathbf{q} \cdot \mathbf{b}),(\mathbf{c},-\mathbf{q} \cdot \mathbf{c}),(0,1),
$$

and the reciprocal lattice then has basis vectors

$$
\left(\mathbf{a}^{*}, 0\right),\left(\mathbf{b}^{*}, 0\right),\left(\mathbf{c}^{*}, 0\right),(\mathbf{q}, 1) .
$$

Therefore, the projection of the reciprocal lattice vectors of the physical space $\phi=0$ is exactly the Fourier module of the modulated phase: $h_{1} \mathbf{a}^{*}+h_{2} \mathbf{b}^{*}+h_{3} \mathbf{c}^{*}+h_{4} \mathbf{q}$.

In 1972 de Wolff showed his approach at the IUCr meeting in Kyoto (de Wolff \& van Aalst, 1972). There he met Janner who talked about the embedding of a vibrating crystal in space-time. The symmetry groups there are generalized magnetic space-time groups. The two realized that the symmetry groups in both cases were the same. (In terms of modulated phases, our groups covered both incommensurate and commensurate phases.) This started a collaboration between the group of de Wolff in Delft and our group in Nijmegen.

Together we worked on the development of the formalism and the notation. This was published in de Wolff et al. (1981) and Janssen et al. (1992). Here we give an overview of the

1972 Kyoto, PM de Wolff: world-lines for modulated atomic positions

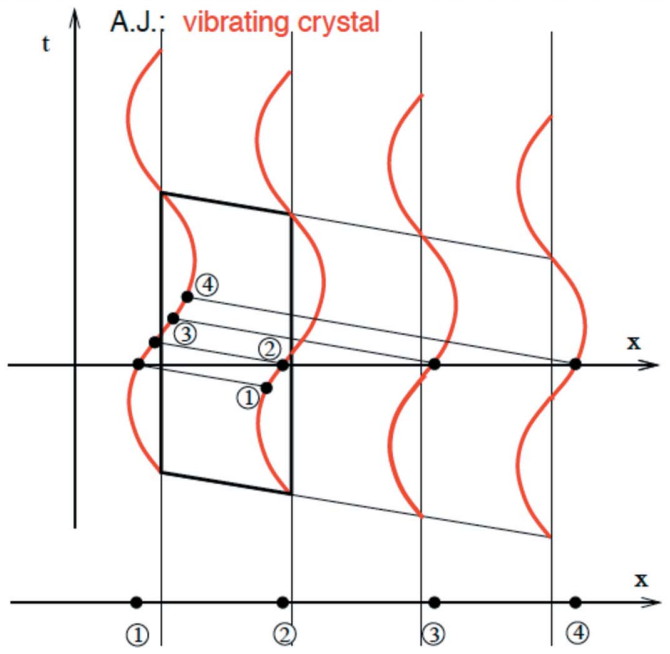

Figure 2

Modulated structure in superspace and vibrating crystals in space-time. For the former the lines are called atomic surfaces, for the latter trajectories. A.J. refers to Aloysio Janner. 
procedure. In general, there is a function (e.g. a density function), $f(\mathbf{r})$, with a Fourier transform consisting of delta peaks. For a one-dimensional modulated structure, the diffraction spots are at the positions $\mathbf{H}=h_{1} \mathbf{a}_{1}^{*}+$ $h_{2} \mathbf{a}_{2}^{*}+h_{3} \mathbf{a}_{3}^{*}+h_{4} \mathbf{q}$. These points form the Fourier module, $M^{*}$, of the diffraction. The density of the compound has a Fourier decomposition.

$$
f(\mathbf{r})=\sum_{\mathbf{H} \in M^{*}} \hat{f}(\mathbf{H}) \exp (i \mathbf{H} \cdot \mathbf{r}) .
$$

One now considers the Fourier module as the projection of a four-dimensional reciprocal lattice: the basis vectors $\mathbf{a}_{i}^{*}$ and $\mathbf{q}$ are the projection of $\left(\mathbf{a}_{i}^{*}, 0\right)$ and $(\mathbf{q}, 1)$. These generate the reciprocal lattice $\Sigma^{*}$. The diffraction spot $\mathbf{H}=\sum_{i} h_{i} \mathbf{a}_{i}^{*}+h_{4} \mathbf{q}$ then is the projection of the four-dimensional vector $\left(\mathbf{H}, h_{4}\right)$. From the aperiodic function $f(\mathbf{r})$, one may construct a lattice periodic structure in four dimensions by

$$
f(\mathbf{r}, t)=\sum_{\mathbf{H} \in M^{*}} \hat{f}(\mathbf{H}) \exp \left(i\left(\mathbf{H} \cdot \mathbf{r}+h_{4} t\right)\right) .
$$

This function has Fourier components on the nodes of the reciprocal lattice that give the spots of the diffraction pattern by projection, and the three-dimensional function is obtained from the four-dimensional one by taking $t=0$ (Fig. 3).

$\gamma-\mathrm{Na}_{2} \mathrm{CO}_{3}$ has a modulated phase with one modulation wavevector. Other compounds have more modulation waves. In general, the Fourier module is given by reciprocal vectors

$$
\mathbf{H}=\sum_{i=1}^{n} h_{i} \mathbf{a}_{i}^{*},
$$

where $n$ is larger than 3 . Now, an $n$-dimensional reciprocal lattice with $n$ basis vectors $\left(\mathbf{a}_{i}^{*}, \mathbf{b}_{i}^{*}\right)$ is introduced. Then a lattice periodic function in $n$ dimensions is obtained by taking the Fourier transform. This gives a function $f\left(\mathbf{r}, \mathbf{r}_{I}\right)$ which has lattice periodicity, with basis vectors $\left(\mathbf{a}_{i}, \mathbf{b}_{i}\right)$. The vectors $\mathbf{a}_{i}$ are three-dimensional and the vectors $\mathbf{b}_{i}$ have the dimension $d=n-3$.

3.3.2. General incommensurate modulated phases. Modulated phases may also occur with more than one modulation wavevector. In general, the spots of the diffraction pattern may be given by

$$
\mathbf{H}=\sum_{i=1}^{n} h_{i} \mathbf{a}_{i}^{*}, h_{i} \text { integers. }
$$

These points form the Fourier module (or, in mathematical terms, the Z-module) of the structure, $M^{*}$. The number of rationally independent basis vectors is the rank of the Fourier module. Again, the points of the Fourier module may be seen as projections of the points of a lattice in $n$ dimensions, $\Sigma^{*}$, with basis vectors $\left(\mathbf{a}_{i}^{*}, \mathbf{b}_{i}^{*}\right)$. The lattice points of $\Sigma^{*}$ are then the points $\left(\mathbf{H}, \mathbf{H}_{\mathrm{I}}\right)$, where the subscript 'I' represents internal. If the modulated phase is given by a density function $\rho(\mathbf{r})$ in three dimensions, it may be decomposed as

$$
\rho(\mathbf{r})=\sum_{\mathbf{H} \in M^{*}} \hat{\rho}(\mathbf{H}) \exp (i \mathbf{H} \cdot \mathbf{r}),
$$

and a lattice periodic function in the $n$-dimensional space is defined by

$$
\rho\left(r, r_{\mathrm{I}}\right)=\sum_{H \in M^{*}} \hat{\rho}(H) \exp \left(i\left(H . r+H_{\mathrm{I}} \cdot r_{\mathrm{I}}\right)\right) .
$$

By construction, this is a lattice periodic function with lattice $\Sigma$ in $n$ dimensions. The modulated phase in three dimensions is just the value of the periodic function $\rho\left(\mathbf{r}, \mathbf{r}_{\mathrm{I}}\right)$ on the line $\mathbf{r}_{\mathrm{I}}=$ 0 . The $n$-dimensional space is called superspace. The prefix 'super' has seen a considerable inflation. Here it does not mean something especially good, but the term simply comes from the relation of the three- and $n$-dimensional spaces. The first is the physical space, also called the external space. It is a subspace of the $n$-dimensional space, which consequently is called superspace, just as seen with the relation between a group, a subgroup and a supergroup. As the rank of the Fourier module and the dimension of the corresponding superspace are equal, these terms may be used without distinction.

The lattice periodic function $\rho$ has an $n$-dimensional space group, called a superspace group. Its point group is given by the symmetry of the diffraction pattern. If an orthogonal transformation (rotation, mirror or inversion), $R$, leaves the diffraction pattern invariant, then

$$
R \mathbf{a}_{i}^{*}=\sum_{j=1}^{n} \Gamma^{*}(R)_{i j} \mathbf{a}_{j}^{*}(i=1, \ldots, n) .
$$

The $n \times n$ integer matrix $\Gamma^{*}(R)$ acts on the points of the reciprocal lattice. The action of $R$ on the direct lattice is then given by its conjugate $\Gamma(R)_{i j}=\Gamma^{*}\left(R^{-1}\right)_{j i}$. As main reflections transform onto main reflections, the matrices $\Gamma(R)$ have the form

$$
\Gamma(R)=\left(\begin{array}{cc}
\Gamma_{\mathrm{E}} & 0 \\
\Gamma_{M} & \Gamma_{\mathrm{I}}
\end{array}\right) .
$$

Because all $\Gamma(R)$ form a finite group, they may be further reduced, not necessarily with integers. In general, the $n$ dimensional operators can also be written as
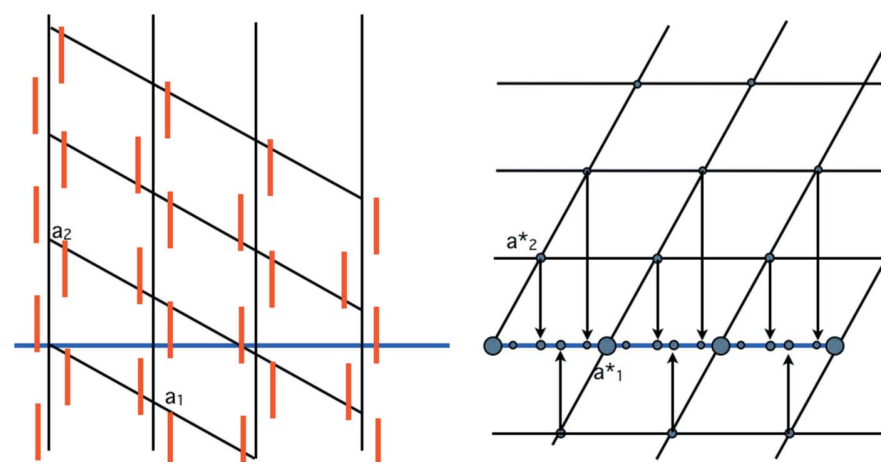

Figure 3

Direct and reciprocal lattice for a modulated phase with a Crenel modulation. The intersection with the physical space (the horizontal line) gives the aperiodic one-dimensional crystal. The projection of the reciprocal lattice is the Fourier module of this structure. 


$$
\Gamma(R) \equiv\left(\begin{array}{cc}
R_{\mathrm{E}} & 0 \\
0 & R_{\mathrm{I}}
\end{array}\right),
$$

which can be written as $\left(R_{\mathrm{E}}, R_{\mathrm{I}}\right)$, a couple of orthogonal transformations, $R_{\mathrm{E}}$ in the three-dimensional physical (or external) space $V_{\mathrm{E}}\left(\right.$ i.e. $\mathbf{r}_{\mathrm{I}}=0$ ), and $R_{\mathrm{I}}$ in the $d$-dimensional internal (or perpendicular) space, $V_{\mathrm{I}}$. The superspace is the direct sum of $V_{\mathrm{E}}$ and $V_{\mathrm{I}}$, and has the dimension $n=3+d$.

In the point-atom approximation, the lattice periodic function in superspace is only non-zero on lines when $n=4$ (see Fig. 2). For $n=5$ it is non-zero on a two-dimensional surface. In general, it is non-zero on an object of dimension equal to that of internal space. In all these cases, they are called atomic surfaces, but they always have the same dimension as $V_{\mathrm{I}}$. They are not necessarily continuous.

Other quantities, like the electron density, are not restricted to $d$-dimensional objects. They are really functions of $n$ variables.

3.3.3. Superspace groups. A general element of a $(3+d)$ dimensional superspace group may be denoted by $g=\left\{\left(R_{\mathrm{E}}, R_{\mathrm{I}}\right),\left(\mathbf{t}_{\mathrm{E}}, \mathbf{t}_{\mathrm{I}}\right)\right\}$. The pairs $\left(R_{\mathrm{E}}, R_{\mathrm{I}}\right)$ form the point group $K$ and the vectors $\left(\mathbf{t}_{\mathrm{E}}, \mathbf{t}_{\mathrm{I}}\right)$ the corresponding translation vectors. The basis of the lattice in superspace is $\left(\mathbf{a}_{\mathrm{E} i}, \mathbf{a}_{\mathrm{I} i}\right)$ $(i=1, \ldots, n)$.

The $n$-dimensional lattice, $\Sigma$, may be determined from the metric tensor $g_{i j}=\mathbf{a}_{\mathrm{E} i} \cdot \mathbf{a}_{\mathrm{E} j}+\mathbf{a}_{\mathrm{I} i} \cdot \mathbf{a}_{\mathrm{I} j}$ which has to satisfy the equation

$$
g=\Gamma(R) g \Gamma(R)^{\mathrm{T}}, \text { for all } R \in K .
$$

Here superscript Trepresents transpose. In general, the tensor $g$ has a number of free parameters. One of them is the length scale in internal space, which has no physical meaning, e.g. for a one-dimensional modulated chain with wavevector $\mathbf{q}$ and lattice constant $a$ of the basic structure, the basis of the lattice in direct superspace consists of $(a,-c q a)$ and $(0, c)$, for the reciprocal lattice $(1 / a, 0)$ and $(\mathbf{q}, 1 / c)$. One may then choose $c=1$.

Formalism and notation were developed in close collaboration with Pim de Wolff. A list of all $(3+1)$-dimensional superspace groups was published in de Wolff et al. (1981), and after some corrections (Yamamoto et al., 1985) in International Tables for Crystallography (Vol. C). All generalized magnetic space-time groups (Fast \& Janssen, 1968) are also $(3+1)$ dimensional superspace groups. The lists differ because of a difference in the definition of equivalence. This means that some magnetic space-time groups are non-equivalent as group extensions but equivalent as superspace groups. We come back to the question of equivalence later. Other lists of $(3+d)$ dimensional superspace groups followed. Yamamoto put four-, five- and six-dimensional superspace groups on his website. Recently these superspace groups were recalculated (Stokes et al., 2011, 2013). As the number of superspace groups grows rapidly with higher dimensions, it becomes impractical to publish the lists in volumes like the International Tables for Crystallography. Moreover, on a website, the data can be manipulated with appropriate software, e.g. to check other settings.
A superspace group for modulated structures may be given by the space group of the basic structure, and information about the internal space transformations accompanying the elements of the basic space group. The basic space group is given by the usual symbol from the International Tables for Crystallography (Vol. A). Next is the information about the modulation wavevectors. We start with the case where the rank is four. Then there is just one modulation wavevector, its components are either rational or irrational. For the latter the symbols $\alpha, \beta$ and $\gamma$ are used. The symbol for the basic space group consists of symbols for some generators, rotations, mirrors, glide planes etc. Each of them represents an orthogonal transformation $R$, which in the superspace group is combined with $R_{\mathrm{I}}=\epsilon= \pm 1$. If $\epsilon=-1$, the corresponding translation $t_{\mathrm{I}}$ can be annihilated by a shift of origin, when $\epsilon=+1$, the values of $t_{I}$ are rational, with denominators $1,2,3$, 4 or 6: $t_{\mathrm{I}}=0, \frac{1}{2}, \pm \frac{1}{3}, \pm \frac{1}{4}$ or $\pm \frac{1}{6}$. These are given by a symbol, $0, s, t, q$ or $h$, one for each symbol of the space group. For example, the superspace group of dimension 4 (or $3+1$ to indicate that it is a superspace group) with generators $\left\{\left(m_{x}, 1\right)\right.$, $\left.\left(0,0,0, \frac{1}{2}\right)\right\},\left\{\left(m_{y}, 1\right),\left(\frac{1}{2}, 0, \frac{1}{2}, 0\right)\right\}$ and $\left\{\left(m_{z},-1\right),\left(\frac{1}{2}, 0,0,0\right)\right\}$ is denoted by Pmna $(00 \gamma) s 00$.

The modulation wavevector $\mathbf{q}$ may be decomposed into two components, one with irrational numbers, and one with rational numbers: $\mathbf{q}=\mathbf{q}^{\mathrm{c}}+\mathbf{q}^{\mathrm{i}}$. The irrational part is given by

$$
\mathbf{q}^{\mathrm{i}}=\frac{1}{N} \sum_{R} \epsilon(R) R \mathbf{q},
$$

where the sum is over all elements of the Laue group, which has $N$ elements. The remaining part, $\sigma^{r}$, has purely rational components. If they are not integers one may make them integers by a basis transformation. A non-zero $\sigma^{r}$ thus corresponds to a centering of the lattice in superspace.

A first proposal for the notation used two-line symbols. If the space group of the basic structure is Pmna, and the wavevector, $\mathbf{q}$, is $\gamma \mathbf{c}^{*}$, the values of $\epsilon$ corresponding to $m, n$ and $a$ are 1,1 and -1 , respectively. The rational component of $\mathbf{q}$ is 0 . Then the symbol would be

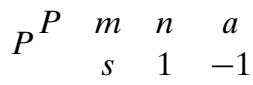

because the translation in internal space with the $\mathrm{x}$-mirror is $\frac{1}{2} e_{4}$, and is indicated by 's' under the symbol ' $m$ '. A non-zero rational component of $\mathbf{q}$ leads to a centering in superspace. Then the first ' $\mathrm{P}$ ' is replaced by a symbol for the centering in question. However, this notation turned out to be impractical because of typographical problems. Therefore, we do not go into detail here.

For higher-dimensional superspace groups the components of the modulation wavevectors are also given, but only for the independent ones, e.g. for a basic space group $P 4 m m$ with rank 5 , and modulation wavevectors $\mathbf{q}_{1}=(\alpha, 0,0)$ and $\mathbf{q}_{2}=(0, \alpha, 0)$, the superspace group could be $\operatorname{P4mm}(\alpha 00)$, because $\mathbf{q}_{1}$ is transformed into $\mathbf{q}_{2}$ by the fourfold rotation.

The formalism is easily extended to higher-dimensional superspace groups. The matrix $\Gamma_{\mathrm{I}}$ in equation (15) is $d \times d$, and the matrix $\Gamma_{M}$ is $3 \times d$. The modulation wavevectors may 
be expressed in the basis vectors of the basic structure if irrational numbers are allowed: $\mathbf{a}_{3+j}^{*}=\sum_{i=1}^{3} \sigma_{j i} \mathbf{a}_{i}^{*}$. The matrix $\sigma$ then may be decomposed into a rational and an irrational part

$$
\sigma=\sigma^{i}+\sigma^{r}, \text { with } \sigma^{i}=\frac{1}{N} \sum_{R} \Gamma_{\mathrm{I}}(R) \sigma \Gamma_{\mathrm{E}}\left(R^{-1}\right)
$$

This is the generalization of equation (18).

If $\sigma^{r}$ does not vanish, the lattice in superspace may be considered as a centering of a simpler lattice. Consider the superspace group $\operatorname{Pm} 2 m\left(\frac{1}{2} 0 \gamma\right)$. A basis for the Fourier module consists of the four reciprocal lattice vectors, $\mathbf{a}^{*}, \mathbf{b}^{*}, \mathbf{c}^{*}$ and $\mathbf{q}=\frac{1}{2} \mathbf{a}^{*}+\gamma \mathbf{c}^{*}$. With respect to this basis, the indices are $h_{1}, h_{2}, h_{3}, h_{4}$. The basis for the centered lattice may be chosen to be $\mathbf{a}^{*}, \mathbf{b}^{*}, \mathbf{c}^{*}$ and $\gamma \mathbf{c}^{*}$. For this basis the indices are $H_{1}, H_{2}, H_{3}$ and $H_{4}$ with centering conditions $H_{1}+H_{4}=$ even. These centering conditions are given for $(3+1)$-dimensional superspace groups in Vol. C of the International Tables for Crystallography. The matrices $\Gamma_{M}(R)$ vanish for this basis. The matrices for $m_{x}$ and $m_{z}$ on the primitive and the centered bases then are

$$
\begin{aligned}
& m_{x}(1)=\left(\begin{array}{cccc}
-1 & 0 & 0 & 0 \\
0 & 1 & 0 & 0 \\
0 & 0 & 1 & 0 \\
1 & 0 & 0 & 1
\end{array}\right) \equiv\left(\begin{array}{cccc}
-1 & 0 & 0 & 0 \\
0 & 1 & 0 & 0 \\
0 & 0 & 1 & 0 \\
0 & 0 & 0 & 1
\end{array}\right) \\
& m_{z}(\overline{1})=\left(\begin{array}{cccc}
1 & 0 & 0 & 0 \\
0 & 1 & 0 & 0 \\
0 & 0 & -1 & 0 \\
1 & 0 & 0 & -1
\end{array}\right) \equiv\left(\begin{array}{cccc}
1 & 0 & 0 & 0 \\
0 & 1 & 0 & 0 \\
0 & 0 & -1 & 0 \\
0 & 0 & 0 & -1
\end{array}\right) .
\end{aligned}
$$

The introduction of superspace groups gives rise to the question as to what to consider as equivalent groups. For three-dimensional space groups, a first definition considers space groups as equivalent if they are affine conjugated. This means that there is a Euclidean transformation, $g_{E}=\{R \mid \mathbf{t}\}$ (i.e. an orthogonal transformation combined with a translation), such that for every element, $g$, of one group the conjugate $g_{E} g g_{E}^{-1}$ is an element of the other group. Bieberbach has shown that this is equivalent with a definition which calls space groups equivalent if they are isomorphic. This definition gives 219 space groups in three dimensions. A stronger definition calls space groups equivalent if they are conjugate with a Euclidean transformation with orthogonal transformation, $R$, with a determinant equal to +1 . For superspace groups, one has to distinguish main reflection and satellites in the Fourier module. Both groups can be brought into a form with point group elements as in equation (15). Two superspace groups are then equivalent if they are conjugated with a Euclidean transformation where the orthogonal element has the same shape as the matrices of equation (15). For example, the superspace groups $P m 11(\alpha 00)$ and $P 112(00 \gamma)$ are equivalent as four-dimensional groups. They have the point groups $m(\overline{1})$ and 2(1), respectively. However, as superspace groups they are non-equivalent, because the conjugating element interchanges main reflections and satellites.

3.3.4. Other incommensurate modulated crystals. The incommensurate phase of dehydrated $\mathrm{Na}_{2} \mathrm{CO}_{3}$ turns out to be an intermediate phase. The high-temperature hexagonal phase has a transition to a monoclinic phase followed by one to the incommensurate modulated phase with wavevector $\mathbf{q}=(\alpha, 0, \gamma)$. The values of $\alpha$ and $\gamma$ change with temperature and at $T=130 \mathrm{~K}$ they lock-in to rational values $\alpha=1 / 6, \gamma=1 / 3$. This means that the low-temperature phase is again lattice periodic, and is a superstructure of the high-temperature phase.

After the discovery of $\mathrm{Na}_{2} \mathrm{CO}_{3}$ a large number of such incommensurate phases were found. Also another type of modulated phase was found, where the positions of the atoms were lattice periodic, but where the probability to find a certain species of atom depends on the unit cell. This is called a substitution modulation. Among the most studied incommensurate modulated structures could be mentioned $\mathrm{K}_{2} \mathrm{SeO}_{4}$, TTF-TCNQ, the family of $A_{2} B X_{4}$ compounds (e.g. $\left.\mathrm{Rb}_{2} \mathrm{ZnBr}_{4}\right)$ and biphenyl. Most of them have a one-dimensional modulation $(d=1, n=4)$ and are intermediate phases (Fig. 4). Also, in terms of total volume these phases are not rare: many minerals show an incommensurate modulation. Thus, a substantial part of the earth's crust consists of incommensurate crystals.

\subsection{Diffraction}

For a conventional three-dimensional crystal, the diffraction is determined by the structure factor, $F(\mathbf{H})$.

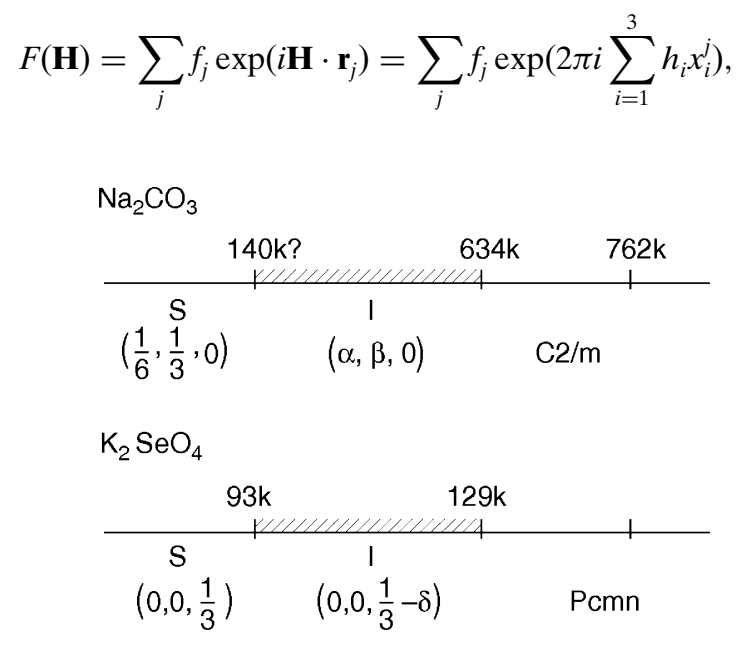

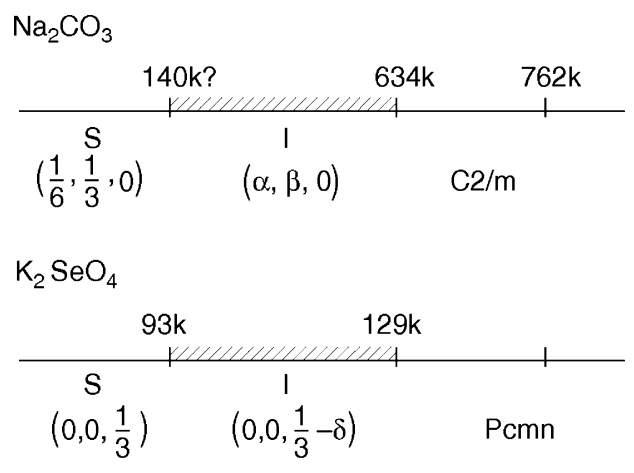

TTF-TCNQ

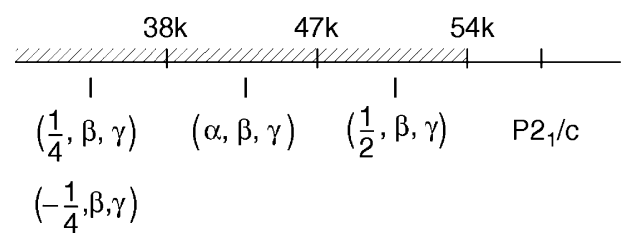

Figure 4

Three examples of incommensurate phases (the setting of $\mathrm{Na}_{2} \mathrm{CO}_{3}$ differs from that in $\$ 3.1$ ). 
where the sum over $j$ is a sum over the particles in the unit cell, at the positions $\mathbf{r}_{j}$, and $f_{j}$ the atomic structure factor. For an incommensurate modulated crystal, $F(\mathbf{H})$ is equal to the function $F\left(\mathbf{H}, \mathbf{H}_{\mathrm{I}}\right)$ because of the one-to-one correspondence between the vectors $\mathbf{H}$ of the Fourier module, and the vectors $\left(\mathbf{H}, \mathbf{H}_{\mathrm{I}}\right)$ of the $n$-dimensional reciprocal lattice. A difference with the three-dimensional case is that the number of atoms in the $n$-dimensional unit cell is infinite, and therefore the sum must be replaced by a sum over the atomic surfaces, $\Omega_{j}$, and an integration over these atomic surfaces. de Wolff derived the formula for a one-dimensional modulation

$$
F(\mathbf{H})=\sum_{j}\left[\exp \left(2 \pi i \sum_{i=1}^{3} h_{i} x_{i}^{j}\right) \int_{0}^{1} f_{j}\left(x_{4}^{j}\right) \exp \left(2 \pi i h_{4} x_{4}^{j}\right) \mathrm{d} x_{4}^{j}\right]
$$

for a displacive modulation and a more complicated expression for a substitution modulation. For a general modulation, the formula becomes

$$
F(\mathbf{H})=\sum_{j} f_{j} \int_{\Omega_{j}} \exp \left(i\left(\mathbf{H} \cdot \mathbf{r}_{j}\left(r_{\mathrm{I}}\right)+\mathbf{H}_{\mathrm{I}} \cdot \mathbf{r}_{\mathrm{I}}\right)\right) \mathrm{d} \mathbf{r}_{I} .
$$

A similar expression was derived in Yamamoto (1980).

Extinction rules for diffraction peaks also follow from the superspace group, as in three dimensions. To see this, one transforms the action of a superspace group element, $g$, to reciprocal space. In direct space a density transforms under $g$ as follows

$$
T_{g} f\left(\mathbf{r}, \mathbf{r}_{\mathrm{I}}\right)=f\left(R^{-1}(\mathbf{r}-\mathbf{t}), R_{\mathrm{I}}^{-1}\left(\mathbf{r}_{\mathrm{I}}-\mathbf{t}_{\mathrm{I}}\right)\right), g=\left\{\left(R, R_{\mathrm{I}}\right),\left(\mathbf{t}, \mathbf{t}_{\mathrm{I}}\right)\right\} .
$$

Then the action on the Fourier transform is given by

$$
T_{g} \hat{f}\left(\mathbf{H}, \mathbf{H}_{\mathrm{I}}\right)=\exp \left(-i\left(\mathbf{H} \cdot \mathbf{t}+\mathbf{H}_{\mathrm{I}} \cdot \mathbf{a}_{\mathrm{I}}\right)\right) \hat{f}\left(R^{-1} \mathbf{H}\right) .
$$

Applying this to the scattering factor, this means that a spot left invariant by $R$ vanishes if the exponent of the prefactor is not a multiple of $2 \pi$. This shows the importance of introducing the superspace for structure determination.

\subsection{The structure of incommensurate modulated phases}

The first step in the structure determination of an incommensurate modulated phase is the determination of its superspace group. This can be done by the following steps.

(i) First step. Determine the Laue group of the diffraction pattern.

(ii) Second step. Choose a basis for the basic structure according to International Tables for Crystallography (Vol. A), and extend its reciprocal basis to a basis of the Fourier module.

(iii) Third step. Determine the space group of the average structure, obtained from the main reflections.

(iv) Fourth step. Determine the Bravais lattice type in superspace, using the observed extinctions. This also implies the choice of a conventional cell.

(v) Fifth step. Determine the possible superspace groups associated with the space group of the basic structure are compatible with the observed extinctions.
The following step is the determination of the modulation functions. These functions are restricted by the condition that the structure should be invariant under the superspace group. Then the admissible modulation functions should be parametrized, and a limit on the parameters should be imposed, because the space of admissible functions is still of infinite dimension. The parameters can be chosen for a certain class of functions, for example sinusoidal functions or Crenel functions (which are stepwise constant functions).

The final step is the determination of the values of the parameters that agree best with the observed diffraction intensities, using the expressions for the structure factor, as given in $\$ 3.4$.

Examples of incommensurate modulated phases are:

(i) $\mathrm{K}_{2} \mathrm{SeO}_{4}$. This was one of the first incommensurate phases for which a soft mode has been found which leads to the incommensurate phase transition.

(ii) $\mathrm{Rb}_{2} \mathrm{ZnBr}_{4}$ and similar. One of a family of $A_{2} B X_{4}$ compounds with an intermediate incommensurate phase.

(iii) TTF-TCNQ. The three modulated ordered phases in tetrathiafulvalene-tetracyanoquinodimethane may be described in terms of four- and five-dimensional superspace groups (Bak, 1976; Bak \& Janssen, 1978). The $47 \mathrm{~K}$ transition is driven by a soft excitation polarized in the fourth 'phase' direction.

(iv) $\mathrm{ThBr}_{4} \cdot \mathrm{ThBr}_{4}$ has an incommensurate phase induced by a soft mode which becomes unstable at $T=95 \mathrm{~K}$. The wavevector $\mathbf{q} \simeq 0.310$ does not change with temperature, whereas usually the wavevector moves to a rational value at the lock-in transition.

(v) $\mathrm{NaNO}_{2} \cdot \mathrm{NaNO}_{2}$ is an occupational modulated phase where the $\mathrm{NO}_{2}$ triangles may have two orientations. The probability of finding one of these orientations at a given site is an incommensurate probability wave.

(vi) AuCu-II. An alloy where the positions of the atoms occupy sites of a b.c.c. (body-centred cubic) lattice. The probability to find $\mathrm{Au}$ in the position (000) or in $\left(\frac{1}{2} \frac{1}{2} \frac{1}{2}\right)$ is an incommensurate periodic function along one of the axes.

(vii) BCCD (betaine calcium chloride dihydrate; Ao et al., 1989). BCCD has a large number of incommensurate phases in the temperature-pressure phase diagram. It is very similar to the phase diagram in Fig. 37.

(viii) Biphenyl. Biphenyl has a phase diagram with a phase transition to a phase of rank five. The wavevectors are $\pm \mathbf{q}_{1} \pm \mathbf{q}_{2}$ in a plane. At lower temperature, there is a partial lock-in transition: the rank becomes 4 with modulation wavevectors $\pm \mathbf{q}$. This is called a partial lock-in, because one of the vectors $\mathbf{q}$ and $\mathbf{q}_{2}$ becomes rational (0). No full lock-in has been found at even lower temperatures.

\section{Other aperiodic crystals}

\subsection{Incommensurate magnetism}

Before the discovery of the incommensurate phase $\gamma$ $\mathrm{Na}_{2} \mathrm{CO}_{3}$ a periodic magnetic structure was observed, where 
the periodicity did not match that of the underlying crystal. The magnetic moments are given by $\mathbf{M}(\mathbf{n})=\mathbf{M}_{0} \cos (\mathbf{q} \cdot \mathbf{n})$, where $\mathbf{q}$ does not have rational components with respect to the basis of the reciprocal lattice. An explanation was given by Enz (1960), and this was later worked out by Elliot (1965). For their explanation they consider chains of magnetic moments with interactions between first and second neighbors (see §5.7).

The magnetic moments may be characterized with representations of the space group of the lattice. However, as we have seen, this is analogous to a description using superspace. An incommensurate magnetic structure will, generally, be given by

$$
\mathbf{M}(\mathbf{n})=\sum_{\mathbf{H} \in M^{*}} \hat{\mathbf{M}}(\mathbf{H}) \exp (i \mathbf{H} \cdot \mathbf{n}),
$$

where $M^{*}$ is the Fourier module as in equation (11). Then the magnetic structure in superspace is given by

$$
\mathbf{M}\left(\mathbf{n}, \mathbf{r}_{\mathrm{I}}\right)=\sum_{\mathbf{H} \in M^{*}} \hat{\mathbf{M}}(\mathbf{H}) \exp \left(i\left(\mathbf{H} \cdot \mathbf{n}+\mathbf{H}_{\mathrm{I}} \cdot \mathbf{r}_{\mathrm{I}}\right)\right) .
$$

Just as three-dimensional crystals with magnetic moments are described using magnetic groups, magnetic superspace groups may be introduced (Janner \& Janssen, 1980). The action of an element of the magnetic superspace group, which is either $g$ or $g \theta$ ( $\theta$ is the time reversal), on the magnetic structure is

$$
T_{g} \mathbf{M}\left(\mathbf{n}, \mathbf{r}_{\mathrm{I}}\right)=\operatorname{Det}(R) R \mathbf{M}\left(R^{-1}(\mathbf{n}-\mathbf{t}), R_{\mathrm{I}}^{-1}\left(\mathbf{r}_{\mathrm{I}}-\mathbf{t}_{\mathrm{I}}\right)\right),
$$

and an additional minus sign for $g \theta$. Four-dimensional magnetic point and space groups are discussed in Souvignier (2006). They are related to the color point and space groups where Euclidean transformations are combined with permutations of a set distinguished by colors. The colors form a finite group. When there are only two colors the groups are blackand-white: each element of a point group or space group is paired with either an identical permutation or a permutation of two elements. These can be identified with magnetic groups.

As mentioned before, these magnetic structures may also be described using representations of the underlying space group, just as for displacive modulations. A recent review is given in Perez-Mato et al. (2012). When one considers the (real) magnetic moments, the relevant representations are the usual representations. Only when spins are considered in a quantum mechanical context is the use of the more general co-representations needed (see also Janssen, 2014).

Many complicated magnetic phase diagrams with incommensurate magnetic waves occur in rare-earth compounds (Fig. 5). Examples of incommensurate magnetic structures are the following:

(i) Eu-chalcogenides (Janssen, 1972);

(ii) $\mathrm{DyFe}_{4} \mathrm{Ge}_{2}$ (Schobinger-Papamantellos et al., 2006);

(iii) $\mathrm{ErFe}_{4} \mathrm{Ge}_{2}$ (Schobinger-Papamantellos \& Janssen, 2006);

(iv) DySi (Schobinger-Papamantellos et al., 2010).

In principle, the spin-lattice coupling will induce an incommensurate modulation if an incommensurate magnetic wave is present. This means that the magnetic superspace group will be determined by the invariance of both magnetic and nuclear structure. The first is affected by anti-chronous (i.e. containing the time reversal operator $\theta$ ) elements, but $\theta$ leaves the nuclear structure invariant. A simple example is a linear chain with magnetic moments at the atoms. The positions of the atoms are $n a+U \cos \left(q_{1} n a\right)$ and the magnetic moments are $M \cos \left(q_{2} n a\right)$. The embedding in superspace is $\left(n a+U \cos \left(q_{1} n a+t_{1}, t_{1}\right)\right)$ and $M \cos \left(q_{2} n a+t_{2}\right)$. The second is invariant under time reversal in combination with a shift $t_{2} \rightarrow t_{2}+\pi$, but the first is only invariant under this operation when $q_{1}=2 q_{2}$. The magnetic superspace group puts restrictions on the compatible modulations.

\subsection{Incommensurate composites}

Johnson \& Watson (1976) analyzed tetrathiafulvalene (TTF, i.e. $\mathrm{C}_{3} \mathrm{H}_{2} \mathrm{~S}_{2}$ ) with iodine in the approximate composition $(\mathrm{TTF})_{7} \mathrm{I}_{5}$. They found that the ratio between the lattice

a

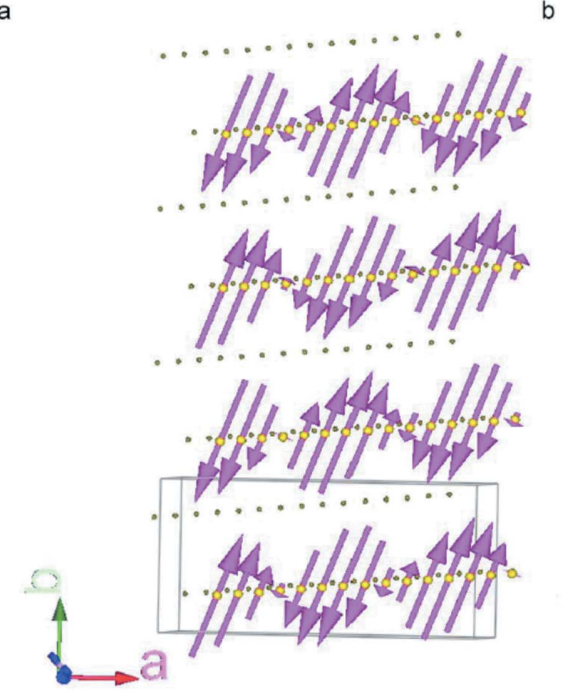

(a)

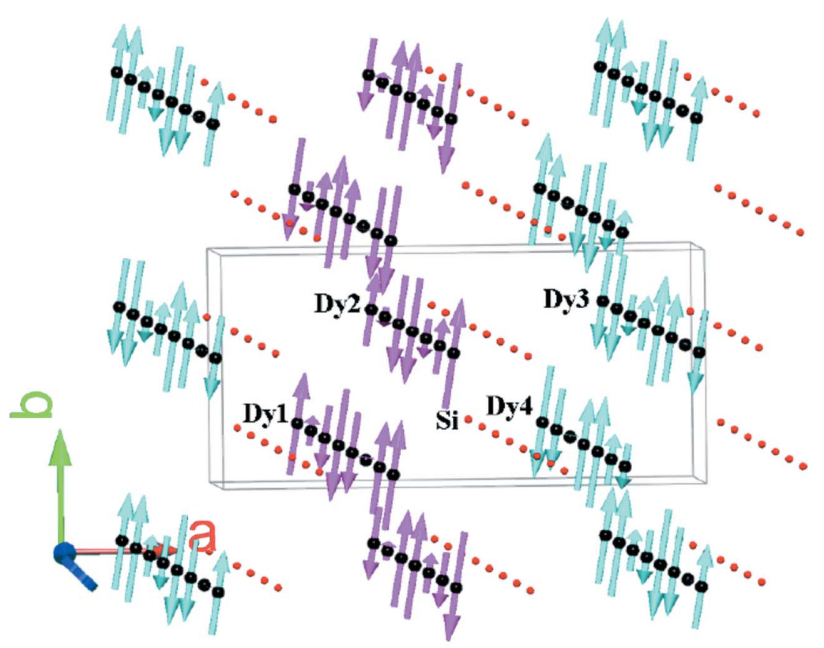

(b)

Figure 5

Incommensurate magnetic structures: $(a)$ DySi at $16 \mathrm{~K}$ (mixture of $\left(0, \frac{1}{2}, \frac{1}{6}\right)$ and $\left.(0, \beta, \gamma)\right) ;(b)$ DySi at $25 \mathrm{~K}, \mathbf{q}=(0, \beta, \gamma)$ (after SchobingerPapamantellos et al., 2010). 
constants of the TTF and I subsystems is not rational. At approximately the same time 'fool's gold' $\left(\mathrm{Hg}_{3-\delta} \mathrm{AsF}_{6}\right)$ was studied and found to have a similar structure (Brown et al., 1974; Cutforth et al., 1976; Pouget et al., 1978). This led us to look for a description of the structure in terms of aperiodic crystals. The formulation used for incommensurate modulated phases had to be adapted to express the special relations in the new class of aperiodic crystals.

An incommensurate composite consists of two or more subsystems which are themselves incommensurate modulated phases, e.g. one subsystem may be a host system with channels in which another subsystem resides. Because of the interaction each subsystem is modulated by the other. In general, there are several subsystems labelled with $v$. Each subsystem has a basic structure with reciprocal basis vectors $\mathbf{a}_{v i}^{*}(i=1, \ldots, 3)$, which shows satellites at the positions $a_{v i}^{*}(i=4, \ldots, n)$. One may then choose a common basis for the diffraction patterns, $\mathbf{a}_{j}^{*}$. Each diffraction spot is given by integer indices with respect to the latter. In particular

$$
\mathbf{a}_{v i}^{*}=\sum_{j=1}^{n} Z_{i j}^{v} \mathbf{a}_{j}^{*} \quad(i=1, \ldots, 3)
$$

However, one may generalize this expression, and include the other peaks, considered as 'satellites'. Then $i$ in the formula runs from 1 to $n$. The vectors with $i=1,2,3$ form the reciprocal basis for subsystem $v$, and the others are satellites, as a consequence of the interaction with the other subsystems. Some of these satellites coincide with the main reflections of the other subsystems. The symmetry of the diffraction pattern is again given by equation (14). Choosing a common basis for all subsystems, including satellites, means that one describes incommensurate composites as a new class of aperiodic crystals (Janner \& Janssen, 1980); see Fig. 6.

\section{Displacive Modulation}

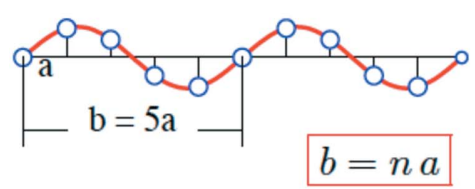

Density Modulation
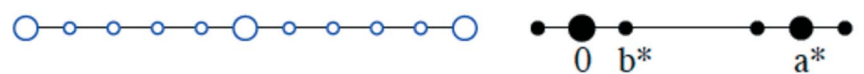

Intergrowth (Composite)
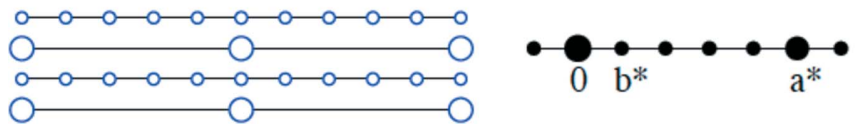

Figure 6

Displacive modulation, occupation modulation and incommensurate composite.
A simple situation occurs when there are two subsystems, the reciprocal basis vectors are $\mathbf{a}_{1}^{*}, \mathbf{a}_{2}^{*}$ and $\mathbf{a}_{3}^{*}$ for the first and $\mathbf{a}_{1}^{*}$, $\mathbf{a}_{2}^{*}$ and $\mathbf{a}_{4}^{*}$ for the second subsystem. The matrices $Z_{i j}^{v}$ then are

$$
Z^{1}=\left(\begin{array}{llll}
1 & 0 & 0 & 0 \\
0 & 1 & 0 & 0 \\
0 & 0 & 1 & 0 \\
0 & 0 & 0 & 1
\end{array}\right) \text { and } Z^{2}=\left(\begin{array}{llll}
1 & 0 & 0 & 0 \\
0 & 1 & 0 & 0 \\
0 & 0 & 0 & 1 \\
0 & 0 & 1 & 0
\end{array}\right)
$$

The general diffraction vector is given by

$$
\mathbf{H}=h_{1} \mathbf{a}_{1}^{*}+h_{2} \mathbf{a}_{2}^{*}+h_{3} \mathbf{a}_{3}^{*}+h_{4} \mathbf{a}_{4}^{*} .
$$

Diffraction spots with $h_{4}=0$ belong to the first (host) system, those with $h_{3}=0$ belong to the second (guest) system, those with $h_{3}=h_{4}=0$ are common spots. It should be noted that the contribution to a 'host' spot does not completely originate from the atoms of the host system, because the modulation of the guest system has these spots as well as modulation vectors.

The reciprocal basis $\mathbf{a}_{v i}^{*}$ determines the direct basis $\mathbf{a}_{v i}$. The embedding of an incommensurate composite is the same as for modulated phases [equation (9)]. Again, the embedding in superspace has an $n$-dimensional superspace group as symmetry. However, there are two differences with the case of modulated phases. The first is that the point group $K_{\mathrm{E}}$ is not restricted to three-dimensional crystallographic groups because there is no basis structure that should be mapped onto itself: some subsystems may be mapped onto each other. Consider, for example, a layered structure where each layer has square symmetry. The odd layers are rotated over $45^{\circ}$ with respect to the even layers. Then the structure has a rank of five and an element of the point group is eightfold rotation. This is not an example from experiment, but it shows that, in principle, non-crystallographic point-group elements are possible.

The second point is the equivalence of superspace groups. One may always choose a subsystem that is a modulated crystal: its Fourier module contains the reciprocal basis of its basic structure and all other diffraction spots are 'satellites'. Then the same symbols for the superspace groups as for modulated crystals can be used. However, by choosing another subsystem as a basis, a symbol is obtained that, according to the equivalence definition, is different from the first symbol. However, the structure is the same and the two symbols should be equivalent in this case. The two groups are in fact isomorphic and this could be used as an equivalence relation, as is also the case for the three-dimensional space groups (when distinguishing 219 and not 230 three-dimensional space groups).

As an example, consider a layered structure. The even layers form a modulated structure with a basic space group of Pbam, the odd layers with space group Cmmm. Choosing the first as the basic structure, the $(3+2)$-dimensional superspace group would be $\operatorname{Pbam}(\alpha \beta, \alpha-\beta)$, and when choosing the second it would be $\operatorname{Cmm}(\alpha 0,0 \beta)$. In the first case the conditions for the peaks are: $h_{2}=$ even for $\left(0, h_{2}, h_{3}, 0, h_{5}\right), h_{1}=$ even for $\left(h_{1}, 0, h_{3}, h, 0\right)$ and $h_{4}+h_{5}=$ even, and in the second case $h_{1}+h_{2}=$ even, $h_{4}=$ even for $\left(0, h_{2}, h_{3}, 0, h_{5}\right)$ and $h_{5}=$ even for $\left(h_{1}, 0, h_{3}, h_{4}, 0\right)$. As superspace groups for modulated structures, these would be non-equivalent, but they should be 
considered as equivalent for composites. The two groups are, however, isomorphic and, consequently, equivalent as fivedimensional space groups. Isomorphism is, in this case, a more appropriate equivalence relation.

Some examples of incommensurate composite crystals are as follows:

(i) $\mathrm{Hg}_{3-\delta} \mathrm{AsF}_{6}$. Fool's Gold' has a basic structure with $\mathrm{AsF}_{6}$ octahedra. In the channels $\mathrm{Hg}$ chains run along the $x$ - and $y$ directions. The lattice constants of the (basic structures of) $\mathrm{AsF}_{6}$ and $\mathrm{Hg}$ subsystems are incommensurate.

(ii) $\mathrm{TTF}_{7} \mathrm{I}_{5-x}$. The TTF frame can be considered as a host system. This is loose enough to allow iodide atoms to fill the channels. The distance between iodide atoms is incommensurate with the lattice constant of the TTF subsystem. TTF and I each form a subsystem, and the rank is 4 .

(iii) $(\mathrm{PbS})_{1+\delta}\left(\mathrm{TiS}_{2}\right)_{2}$. A layered structure with alternating layers of $\mathrm{PbS}$ and $\mathrm{TiS}_{2}$. These layers have basic structures with incommensurate lattice constants.

(iv) Alkane-urea. The host system is urea, formed by helical structures. Alkane molecules are situated in the hexagonal or almost hexagonal channels (Fig. 7). There is a family of alkanes of very different length, where the number of $\mathrm{C}$ atoms in the alkane chains varies from 7 to 24 . Most of them are incommensurate with the urea host. Each of the systems has one or several phase transitions, where the rank sometimes changes (Toudic et al., 2008).

(iv) Adsorbed monolayers. Atomic layers of noble gas adsorbed on a substrate of carbon are two-dimensional aperiodic crystal structures.

(v) Elements with composite structure. It has been found that certain elements under very high pressure form an aperiodic crystal structure. This can be described as a composite with the special property that the two subsystems consist of atoms of the same species (McMahon \& Nelmes, 2006); see Fig. 8.

\subsection{Liquid crystals}

Incommensurate phases may also occur in liquid crystals (Pikin, 1986). If the material consists of rod-like molecules, their directions may be correlated whereas their positions are

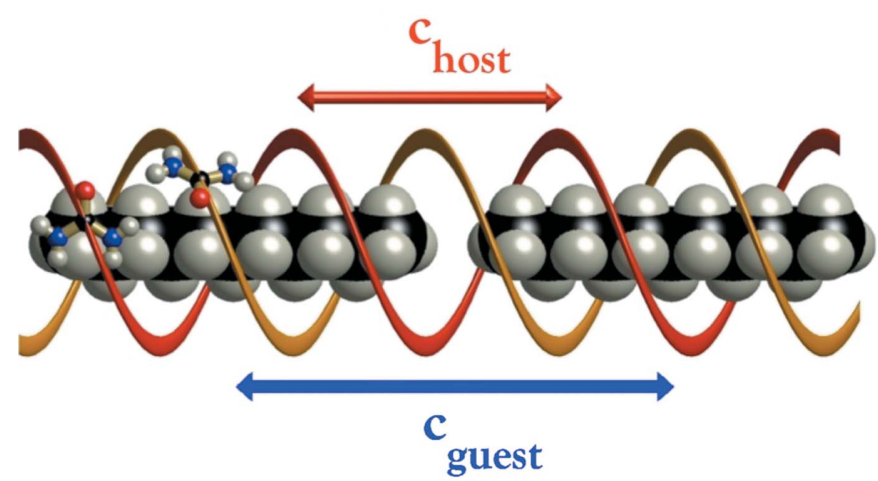

Figure 7

The incommensurate composite alkane-urea (after Toudic et al., 2008). Reproduced with persmission from AAAS. not. If the positions are correlated in the sense that the molecules are concentrated on equidistant planes, the structure is called smectic. When the average orientation of the molecules is perpendicular to these planes, a smectic $A$ phase is obtained; when they are tilted it is a smectic $C$ phase. When the tilted direction varies periodically in a direction perpendicular to the planes, a smectic $C^{*}$ structure is found. In general, the pitch of the screw is incommensurate with the distance between the planes; then one has an incommensurate liquid crystal. In fact, this is similar to an incommensurate spin wave, as discussed previously. Liquid crystals are not crystals in the usual sense, because the systems mentioned above are only ordered perpendicular to the layers, and not in the layers.

\subsection{Quasicrystals}

4.4.1. Discovery. In 1982, Dan Shechtman, from the Technion in Haifa, found (at the National Bureau of Standards in Gaithersburg) that the electron diffraction pattern of rapidly quenched AlMn showed a fivefold symmetry, even the symmetry of an icosahedron. This discovery was not immediately accepted by the community. It took more than 2 years before the publication appeared in Phys. Rev Lett. (Shechtman et al., 1984). Nevertheless, before that, crystallographers had already speculated about the possible existence of such structures (Mackay, 1982). This idea was based on mathematical results on tilings, as will be discussed in the next section.

Shortly after the publication, this type of aperiodic well ordered system was termed a quasicrystal (Levine \& Steinhardt, 1984), although it was not clear what the precise definition was. Per Bak used the term icosahedral incommensurate phase, which expresses the aperiodicity more clearly. Some colleagues use the term 'quasicrystal' exclusively for well ordered structures with a symmetry of the diffraction pattern that is not compatible with three-dimensional lattice periodicity. Others consider the term as an abbreviation of 'quasi-periodic crystals'. The problem with this definition is that according to the mathematical definition of quasi-periodic function, all lattice periodic crystals are also quasi-periodic.

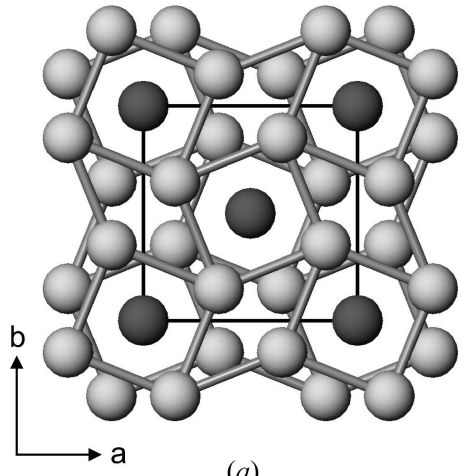

(a)

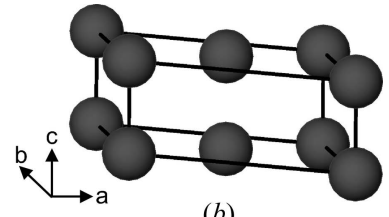

(b)

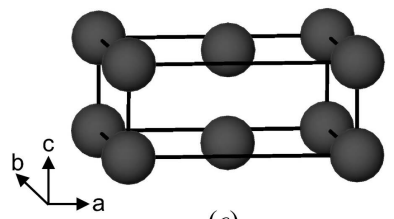

(c)
Figure 8

(a) Structure of $\mathrm{Ba}$ in phase IVa, seen along the $c$ axis. (b) The 'guest atoms' with coexisting monoclinic and tetragonal structure (Reproduced from McMahon \& Nelmes, 2006 with permission from The Royal Society of Chemistry). 


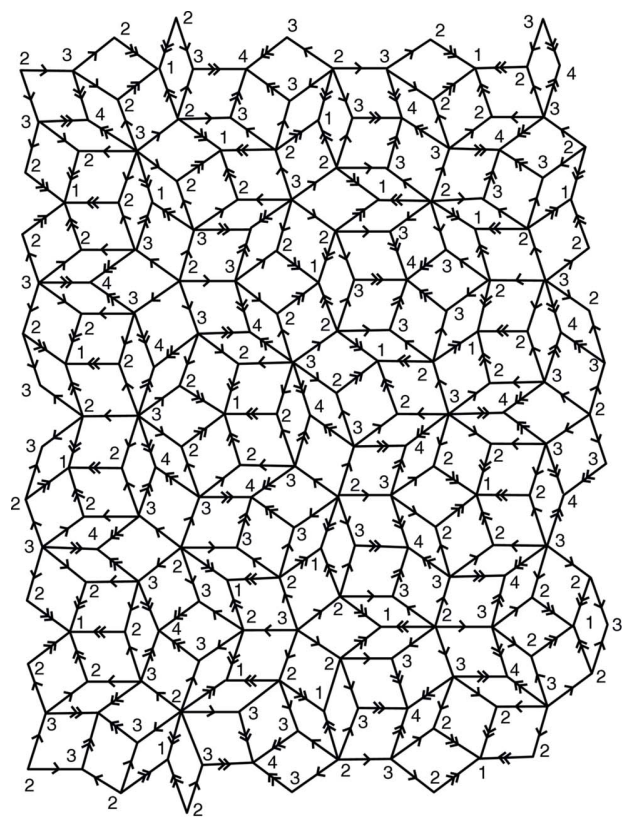

(a)

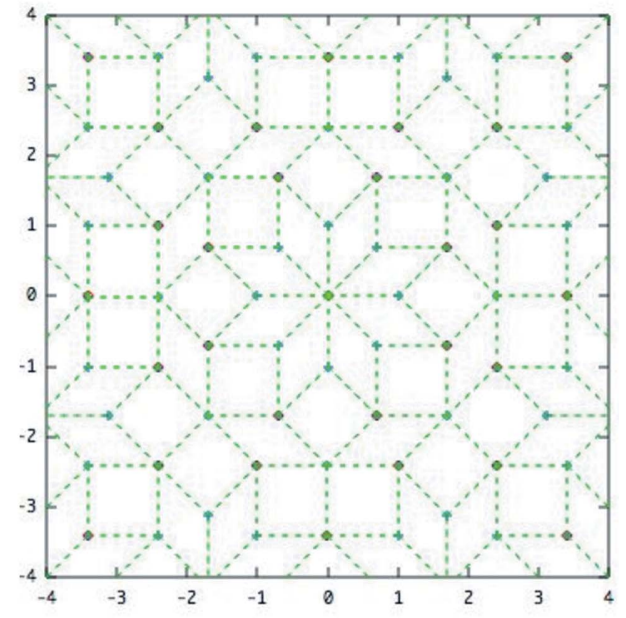

(b)

Figure 9

(a) An example of a Penrose tiling. The arrows show 'matching rules': the edges of the rhombs carry an arrow or a double arrow. Tiles may be next to each other when the arrows match along their common edge. (b) The Ammann-Beenker tiling.

Actually, this was the reason to call the incommensurate modulated crystals 'incommensurate' and not 'quasi-periodic'. The latter term was already introduced by H. Bohr in 1923 . Although there is no consensus about the definition, it is clear that incommensurate phases are not considered to be quasicrystals. This does not refrain people from considering the one-dimensional Fibonacci chain as a model for quasicrystals, which is, strictly speaking, not logical.

The fact that the Fourier module of a quasicrystal has a rank higher than three means that quasicrystals form a new, exciting class of aperiodic crystal. As a consequence, one may use the superspace approach for their description (Janssen, 1986).

Levine \& Steinhardt (1986) discuss the possibility of combining long-range order and non-crystallographic symmetries. This was based on work on fluids with local dodecahedral symmetry.

4.4.2. Mathematical models: tilings. Before the discovery of quasicrystals, mathematicians had studied aperiodic tilings covering the plane with copies of a finite set of 'prototiles' such that there are no overlaps and no spaces between the tiles. A generalization to three dimensions then is simple. If the tiling does not have lattice periodicity it is aperiodic. The best known aperiodic tiling is the Penrose tiling (Penrose, 1974, 1979). One realisation is by means of two prototiles: rhombs with angles of 36 and $72^{\circ}$, respectively, termed skinny and fat rhombs. The mathematics behind the tilings and a method to construct a Penrose tiling was given by de Bruijn (1981). de Bruijn, together with his student Beenker, developed another aperiodic tiling with squares and rhombs with an angle of $45^{\circ}$ as prototiles. This tiling had also been invented, indepen- dently, by Ammann. Therefore, the tiling is known as the AmmannBeenker tiling (see Fig. 9).

The construction method for Penrose tilings, given by de Bruijn, used a five-dimensional space, which can be identified with the superspace used for the description of aperiodic crystals. His method is called the grid method. In the plane five sets of equal-distance parallel lines can be constructed, with the angle between the five sets a multiple of $2 \pi / 5$. Each open space between the lines can then be associated with five integers. These can be used as coefficients of the five vectors of length 1 with an angle $2 \pi m / 5$. This produces the vertices of a Penrose tiling. It is clear that this construction method is closely related to that based on a higher-dimensional superspace.

Mackay used an optical technique to produce a diffraction pattern of the vertices of a Penrose tiling (see Fig. 12a), which has tenfold symmetry and sharp spots. This confirmed the view of some crystallographers that such a tiling has a crystallographic interpretation. The diffraction pattern may be indexed with four indices, as shown in Fig. 12(b). The superspace approach allows the construction of a four-dimensional lattice. The vertices of the tiling should then be the intersections of the two-dimensional physical space and the atomic surfaces. The problem was how to determine these atomic surfaces. This could be done using the results of de Bruijn. It turns out that four atomic surfaces are required in the four-dimensional unit cell (Janssen, 1986); they have the shape of a regular pentagon (see Fig. 10).

It is possible to consider, as de Bruijn did, a five-dimensional lattice. The rank of the Fourier module is four, but, just as four indices can be used for a hexagonal lattice in threedimensions, five indices might be used here for the Penrose tiling. Then there is the relation that the sum of the five basis vectors $(\cos (2 \pi m / 5), \sin (2 \pi m / 5))(m=1, \ldots, 5)$ is zero.

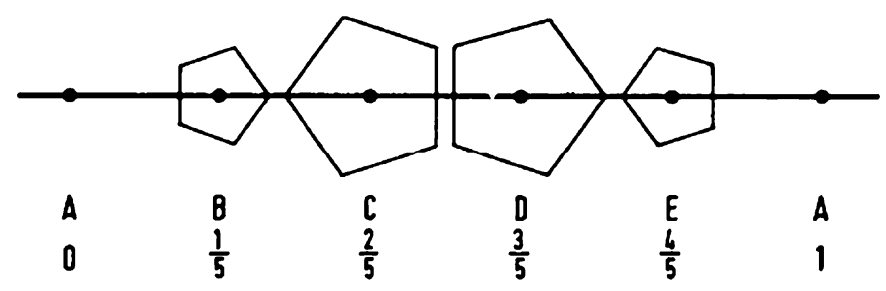

Figure 10

The five pentagons attached to the five Wyckoff positions $\left(\frac{n}{5} \frac{n}{5} \frac{n}{5} 5\right)$ in the unit cell of the decagonal lattice. The pentagon for $n=0$ has shrunk to a point. 
A simpler aperiodic tiling is the Ammann-Beenker tiling which has rank four, and only one atomic surface in the fourdimensional unit cell, a regular octagon, which is the projection of the four-dimensional unit cell on the internal space.

There is a three-dimensional generalization to the twodimensional Penrose tiling. Consider 12 vectors in reciprocal space, pointing to the 12 faces of a dodecahedron. The symmetry of this set is the icosahedral group, $I_{h}$, of the order 120. Six of the 12 vectors are independent, if integers are used as coefficients. Therefore, the three generators of the icosahedral group may be represented by three integer six-by-six matrices. According to group theory, these may be simultaneously brought into the form of the sum of two threedimensional orthogonal matrices. Then one may construct the six six-dimensional reciprocal basis vectors, because if the physical part of a vector goes into another vector in physical space, the transformation of its internal part is given by the corresponding orthogonal matrix in internal space. In that way, a reciprocal lattice is obtained in six dimensions from which the direct lattice is readily obtained. Attach in each lattice point an atomic surface, which in this case is the projection of the six-dimensional unit cell on internal space (Fig. 13). The intersection of this structure in superspace with physical space gives a tiling for which the tiles are oblate and prolate rhombohedra. This is the generalization of the Penrose tiling. It has the icosahedral group of the order 120 as the point-group symmetry for the Fourier module (Fig. 13b). Such an icosahedral tiling in three dimensions was also constructed on the basis of Lie algebra in Kramer \& Neri (1984).

The basis for the diffraction pattern has six basis vectors in three dimensions: $\mathbf{e}_{1}^{*}=(1,1+\tau, 0), \mathbf{e}_{2}^{*}=(-1,1+\tau, 0)$, $\mathbf{e}_{3}^{*}=$ $(0,1,1+\tau), \mathbf{e}_{4}^{*}=(1+\tau, 0,1), \mathbf{e}_{5}^{*}=(1+\tau, 0,-1), \mathbf{e}_{6}^{*}=$ $(0,-1,1+\tau)$, with $\tau=(\sqrt{5}-1) / 2$ (see Fig. 13a). These six reciprocal vectors are the projections of the basis vectors of a six-dimensional reciprocal lattice. The basis for the sixdimensional direct lattice is given by

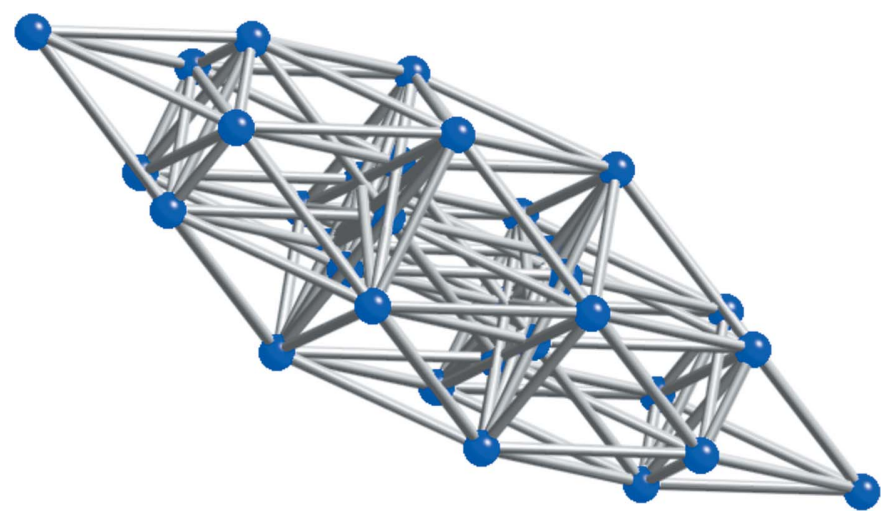

Figure 11

The single atomic surface in the unit cell when the Penrose tiling is embedded in five dimensions. The atomic surface is the interior of the structure, which is left open to show the correspondence with Fig. 10.

$$
\begin{aligned}
& \mathbf{e}_{s 1}=\left(\mathbf{e}_{1}, \mathbf{e}_{1}\right), \mathbf{e}_{s 2}=\left(\mathbf{e}_{2},-\mathbf{e}_{2}\right), \mathbf{e}_{s 3}=\left(\mathbf{e}_{3},-\mathbf{e}_{4}\right) \\
& \mathbf{e}_{s 4}=\left(\mathbf{e}_{4},-\mathbf{e}_{6}\right), \mathbf{e}_{s 5}=\left(\mathbf{e}_{5},-\mathbf{e}_{3}\right), \mathbf{e}_{s 6}=\left(\mathbf{e}_{6},-\mathbf{e}_{5}\right) .
\end{aligned}
$$

There is an arbitrary constant pre-factor in internal space, which here is given as one, and there is an overall pre-factor such that $\mathbf{e}_{s i} \cdot \mathbf{e}_{s j}^{*}=\delta_{i j}$.

There are alternative ways of obtaining aperiodic tilings. One is the so-called cut-and-project method (Duneau \& Katz, 1985; Gähler \& Rhyner, 1986). We illustrate this method on the Fibonacci chain, a one-dimensional aperiodic tiling. A simple way to obtain this is by substitution, as will be explained in §4.4.3. The cut-and-project method starts with a square lattice in two dimensions and draws a line through the origin with a slope $(\sqrt{5}-1) / 2$. Then one considers a band parallel to the line with width $(\sqrt{5}+1) / 2$. All points within this band are projected to the line, and these form a sequence

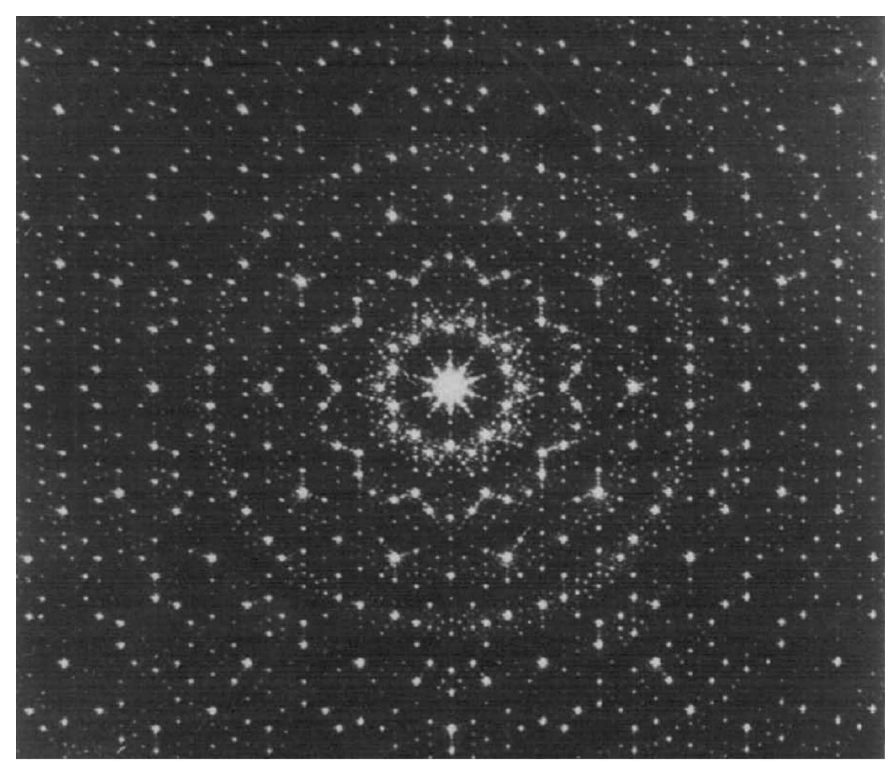

(a)

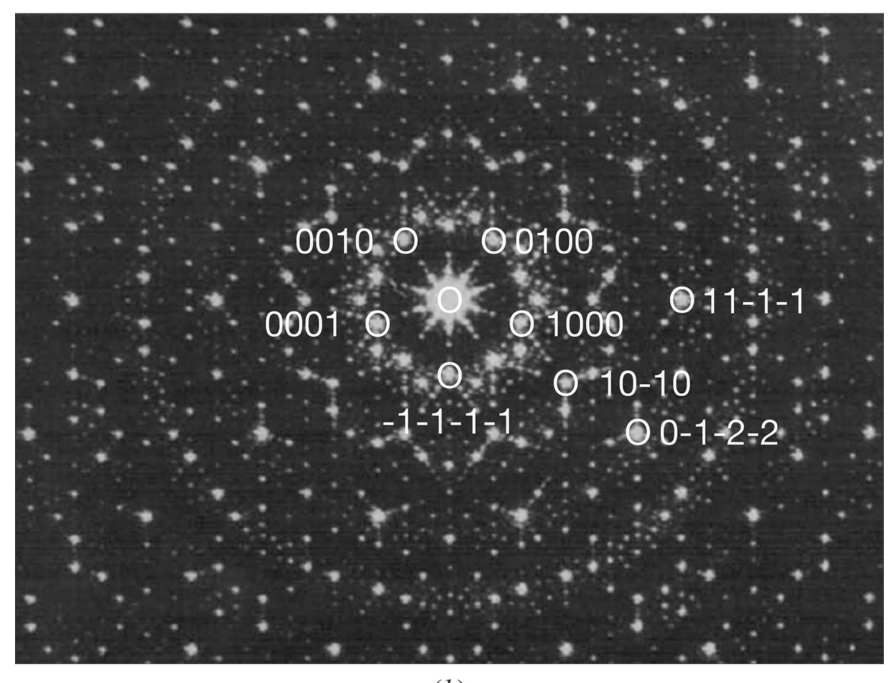

(b)

Figure 12

(a) Optical diffraction from Penrose tiling (afte Mackay, 1982); (b) indexed diffraction spots. 


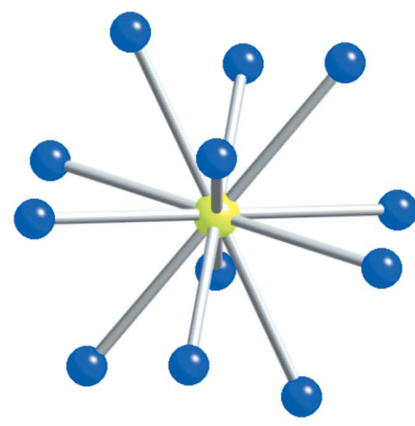

(a)

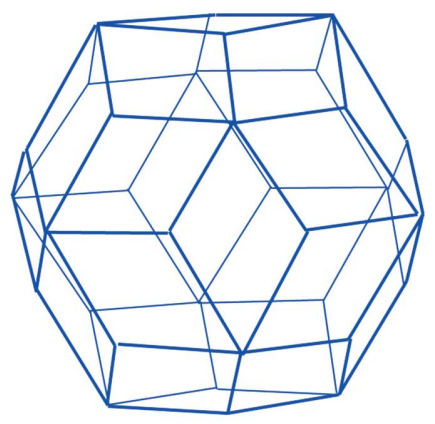

(b)
Figure 13

Three-dimensional icosahedral tiling: $(a)$ six reciprocal basis vectors for the icosahedral tiling and their opposites; $(b)$ atomic surface: a rhombic triacontahedron.

of long and short intervals without lattice periodicity, the socalled Fibonacci chain.

Of course, this is quite similar to a superspace approach. Then the line $y=0$ is the physical space, the two-dimensional superspace has a lattice with basis vectors $(1,-\tau)$ and $(\tau, 1)$ $(\tau=(\sqrt{5}-1) / 2)$ and the atomic surfaces are straight lines of length $1+\tau$. The picture is just rotated (Fig. 14). The difference is that in the cut-and-project method the 'atomic surfaces' are straight, whereas the superspace approach works equally well with curved atomic surfaces. The scale in internal space is not fixed in either of the two cases.

The cut-and-project method has been used to construct the icosahedral three-dimensional tiling discussed above (Duneau \& Katz, 1985; Kalugin et al., 1985a,b; Elser, 1985, 1986; see Fig. 11).

The tilings discussed here were of rank 4 or 6 (icosahedral case). The rank depends on the order of the rotation symmetry. If this order is $1,2,3,4$ or 6 there are periodic tilings. Aperiodic tilings have at least rank 4 in the case of pentagonal, octagonal, decagonal and dodecagonal symmetry ( $C_{n}$ with $n=5,8,10$, and 12 , respectively). From number

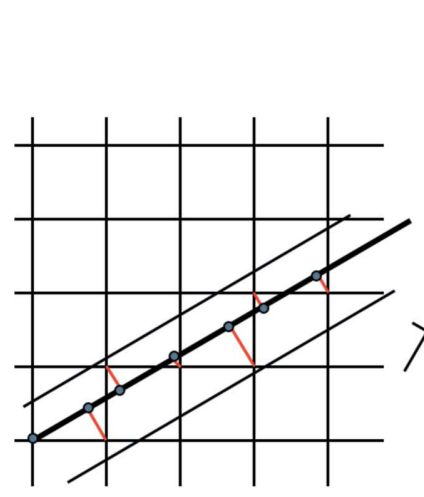

(a) Cut-and-project

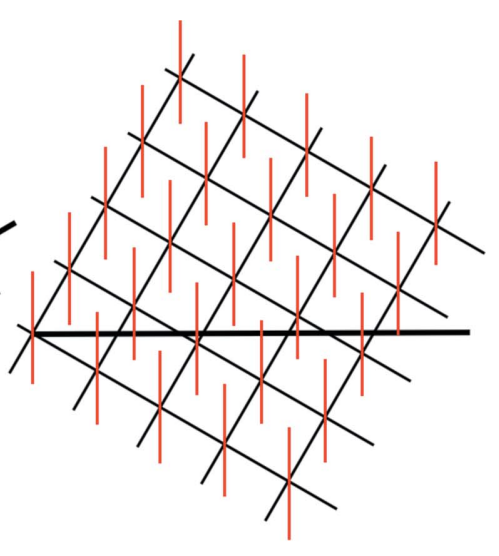

(b) Superspace
Figure 14

The cut-and-project method versus the superspace approach, illustrated on the example of a Fibonacci chain. theory follows a formula for the minimal rank of a rotation of the order $n$. This number is determined by the Euler function $\Phi(n)$ : this is the number of integers $\neq 1$ which are not divisors of $n$, e.g. $\Phi(5)=4$, and consequently, one needs a superspace of dimension four to accommodate a fivefold crystal symmetry.

4.4.3. Quasicrystalline in one dimension. There is still no generally accepted definition of quasicrystal. If one requires rotation symmetry that is not compatible with lattice periodicity in three dimensions, there are no one-dimensional quasicrystals. However, using a more general definition onedimensional structures that have much in common with what are usually called quasicrystals can be studied. The Fibonacci chain is considered to be an example of a one-dimensional quasicrystal. One way to construct such a chain is by substitution. Consider two letters, A and B; start with, for example, $\mathrm{A}$, substitute in every step $\mathrm{A}$ by $\mathrm{B}$, and $\mathrm{B}$ by $\mathrm{AB}$ to give $\mathrm{A}, \mathrm{B}$, $\mathrm{AB}, \mathrm{BAB}, \mathrm{ABBAB}$ etc. Then, construct a chain of intervals of length 1 and $\tau=(\sqrt{5}-1) / 2$ and form a chain with the same order as the letter chain, but instead of A the interval of length 1 , and instead of B the other interval, of length $\tau$. This gives a chain where the end points of the intervals do not have lattice periodicity. However, the Fourier transform has sharp peaks for wavevectors of the form $m_{1}+m_{2} \tau$ (up to a constant). This can be identified as the diffraction pattern of an aperiodic chain of rank two. Therefore, the chain is the intersection of a lattice periodic structure in the $(x, y)$ plane and the line $y=0$. The lattice has basis vectors $(1,-\tau)$ and $(\tau, 1)$ and in the lattice points there is a line segment of length $1+\tau$ parallel to the $y$ axis, which can be considered as a quasicrystal because it is the sequence of points along some line in the Penrose tiling. However, a composite with subsystems with lattice constants $p$ and $q$ ( $p / q$ irrational) in one direction also yields an aperiodic chain along one axis, but there is, generally, no relationship with the quasicrystals of $\$ 4.4 .1$.

4.4.4. Quasicrystalline in two dimensions. There are many quasicrystal compounds that are periodic in one axial direction and aperiodic in the perpendicular plane. The best known family is that of the decagonal quasicrystals. They were the second class of quasicrystals found after the icosahedral AlMn. For a different composition, Bendersky discovered another quasicrystalline structure (Bendersky, 1985). It is

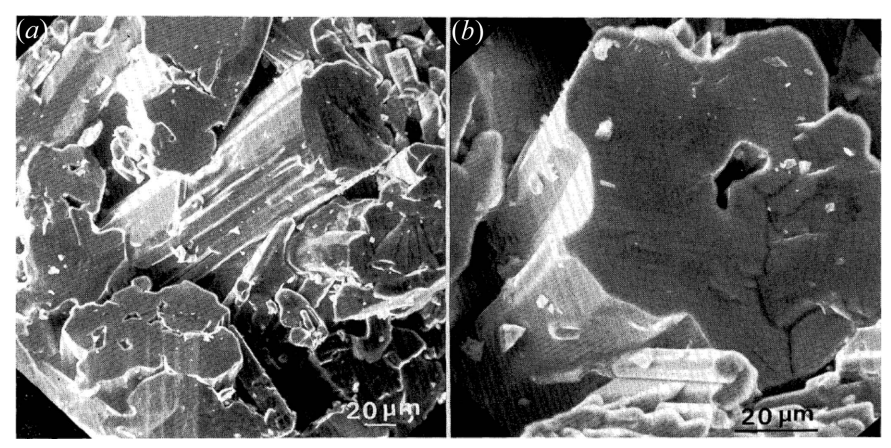

Figure 15

Scanning electron micrographs of solidified decagonal $\mathrm{Al}_{65} \mathrm{Cu}_{15} \mathrm{Co}_{20}$ (after Tsai et al., 1989). 
periodic in one direction and has a tenfold symmetry in the perpendicular plane. It was called the decagonal phase. Much work on this phase has been done by Steurer and his group. Also, compounds with periodicity in one direction and octagonal or dodecagonal symmetry in the perpendicular plane were found. Since two-dimensional pentagonal, octagonal, decagonal and dodecagonal symmetries imply a rank of at least 4 , the rank of these compounds with aperiodic planes is 5 .

Examples of systems with two-dimensional aperiodicity are:

(i) $d$-AlCuCo. The decagonal phase in $\mathrm{AlCuCo}$ was found by Tsai et al. (1989); see Fig. 15.

(ii) $d$-AlCoNi. The structure is a layered structure, with periodicity along one axis. One period consists of an even number of layers $(2,4,6,8)$. However, there are clusters ranging over a number of layers, such that for period 6 a quasiicosahedral symmetry is present. The atomic surfaces of AlCoNi were identified in Cervellino et al. (2002). The determined structure matches very well with STEM pictures of the material (see Fig. 16). The structure has also been discussed in Taniguchi \& Abe (2008). They find a symmorphic superspace group $P \overline{10} \mathrm{~m} 2\left(\overline{10}^{2} \mathrm{~m} 2\right)$ for the layered structure with $2 \mathrm{~nm}$ size clusters. The clusters in this quasicrystal are quite similar to those in an approximant, the so-called Wphase. Using this similarity of constituting clusters is a technique that has also been useful for icosahedral phases.

(iii) $d$ - $\mathrm{ZnMgRE}$ ( $\mathrm{RE}=$ rare earth). A decagonal phase is found in RbMgRE, for which icosahedral quasicrystal structures have also been found. The fivefold surface shows a covering with tiles similar to the Penrose tiling (see Fig. 17).

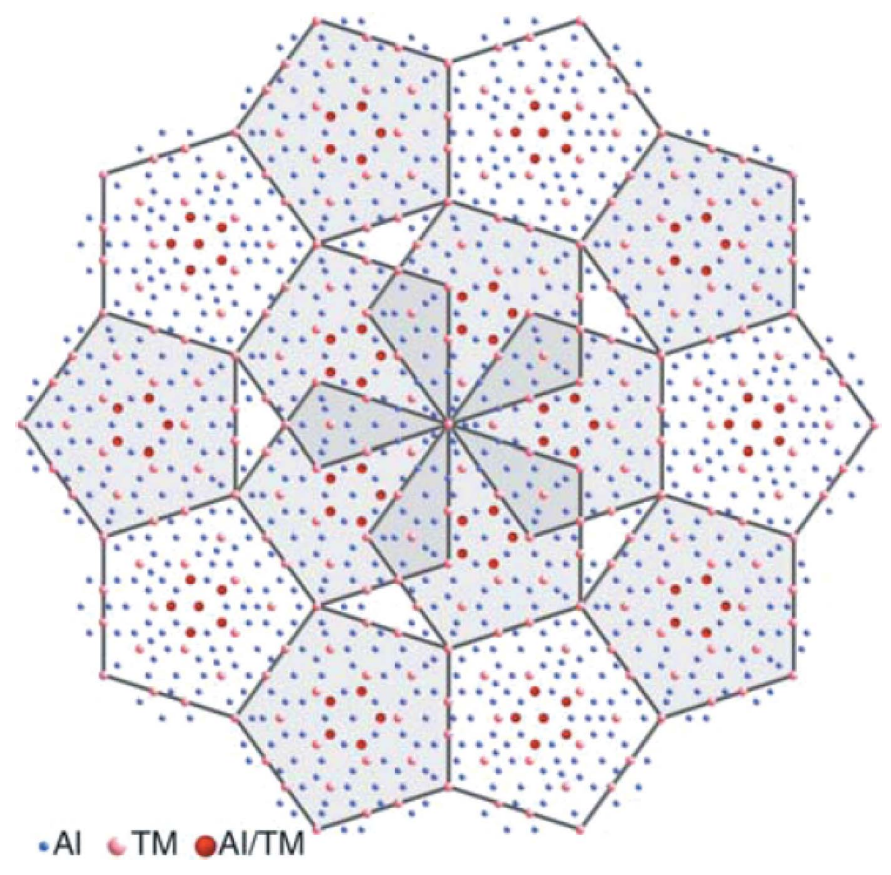

Figure 16

Structure model of d-AlCoNi, with two clusters (shaded and non-shaded; after Steurer \& Deloudi, 2009).
4.4.5. Quasicrystalline in three dimensions. The AlMn quasicrystals found by Shechtman turned out to be unstable. For a long time stable quasicrystals of good quality could not be grown, and this prevented accurate measurements of the physical properties. Now, however, there are several families of quasicrystals having a quality comparable with the best lattice periodic crystals.

Stable quasicrystals (AlCuLi) were grown in 1986 (Dubost et al., 1986). A year later AlCuFe (Tsai et al., 1987) followed. This was the first in a long series of new stable quasicrystals from the group of Tsai.

The diffraction pattern of the quasicrystals which are aperiodic in three directions has the symmetry of the icosahedron, a group of 60 or 120 elements. As an abstract group they are denoted by $I$, respectively $I_{h}$. The (periodic) structures in six dimensions have point groups 532(5232) (abstract group $I$ ) and $5 \overline{3} \mathrm{~m}\left(5^{2} \overline{3} \mathrm{~m}\right)$ (abstract group $I_{h}$ ). These symbols mean that the point group is generated by matrices which are pairs of orthogonal transformations in physical and internal space. For the first generators, these are $R=5$ (a rotation over $72^{\circ}$ ) and $R_{\mathrm{I}}=5^{2}$ (a rotation over $\left.144^{\circ}\right),\left(R=3, R_{\mathrm{I}}=3\right)$ and $\left(R=2, R_{\mathrm{I}}=2\right)$. These point groups leave three different Bravais lattices in six dimensions invariant. The first is a primitive lattice as given for the three-dimensional icosahedral tiling in equation (32). The second is a centering of this lattice, with centering translation $\left(\frac{1}{2} \frac{1}{2} \frac{1}{2} \frac{1}{2} \frac{1}{2}\right)$, and the third is a centering with 32 centering positions, $\left(\frac{1}{2} \frac{1}{2} 0000\right)$ etc. The centering conditions for the I- and F- lattice are $\sum_{i=1}^{6} h=$ even and $h_{i}+h_{j}=$ even (for all $i, j$ ), respectively. These centerings are called P-, I- and F-centerings, respectively.

For incommensurate modulated phases it is usually easy to distinguish the main reflections. For incommensurate composites there is a system of main reflections for each of the subsystems. For quasicrystals this is no longer true. In principle there is a dense set of diffraction peaks, but there is a discrete
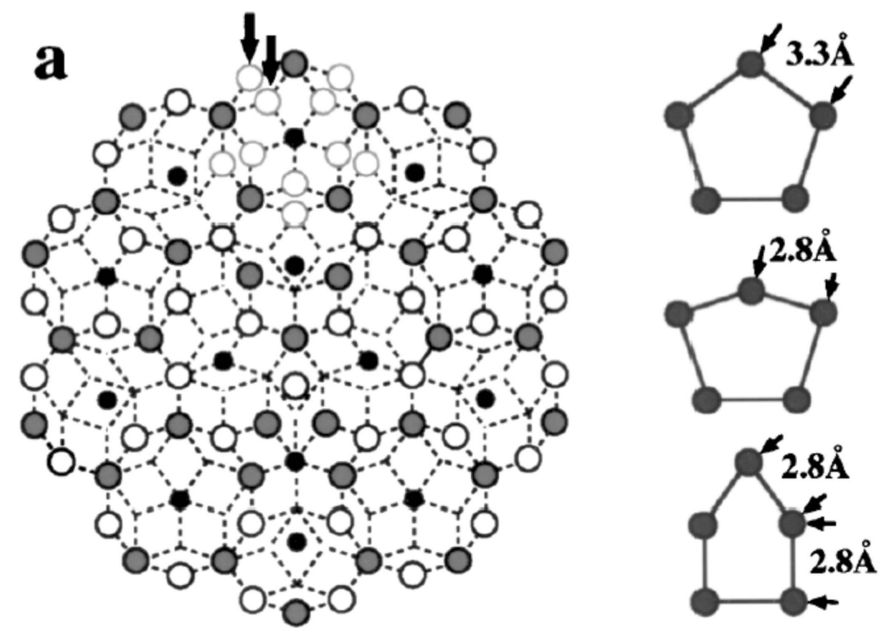

$\mathrm{z}=0, \bullet \mathrm{z}=\mathrm{c} / 4$ and $(3 \mathrm{c}) / 4, \bigcirc \mathrm{z}=\mathrm{c} / 2$ layer

Figure 17

Proposed model for $\mathrm{ZnMgRE}$ and comparison with the Penrose tiling (after Abe et al., 1999). 
set above a certain threshold intensity. Nevertheless, it is not obvious how to proceed to determine a basis for the Fourier module. An additional complication is that there is a common scale factor for an icosahedral quasicrystal such that the position of a diffraction peak is mapped to another when the position is multiplied by that factor (which is $\tau$ or $\tau^{3}$ ). A procedure is discussed by Elser (1985) and Cahn et al. (1986). The basis may be chosen from 12 vectors pointing to the faces of a dodecahedron, and the scale is chosen such that the basis coincides with strong peaks. This gives the six indices $h_{1}, \ldots, h_{6}$ for each peak. Since the six basis vectors may be chosen as $(1+\tau, \pm 1,0),( \pm 1,0,1+\tau)$ and $(0,1+\tau, \pm 1)$, the $x, y$ and $z$ components are $n_{1}+n_{2} \tau$, up to a scale factor. Again, we have six integer indices for each diffraction peak $\mathbf{H}$.

The notation may be simplified by taking the square $H^{2}$, which has length $N+M(1+\tau)$ up to a scale factor. The integers $N$ and $M$ also characterize the spots. However, the notation $\left(h_{1}, \ldots, h_{6}\right)$ is more in agreement with the procedure for other aperiodic crystals. Moreover, the indices $h_{i}$ are not uniquely determined by $N$ and $M$.

The first quasicrystals were P-type. The majority of the newer stable quasicrystals is F-type. Examples of F-type quasicrystals are $\mathrm{AlCuFe}$, AlPdMn and $\mathrm{ZnMgTi}$. Both types occur in $\mathrm{ZnMgTi}$, depending on the precise composition.

Quasicrystals may be described using a superspace by giving the atomic surfaces. This means that the positions of the atomic surfaces in the higher-dimensional unit cell have to be determined, and in addition the shape of these atomic surfaces. It is clear that the structure determination is more complicated than for modulated phases and composites, especially for icosahedral quasicrystals where the atomic surfaces are three-dimensional objects.

In three dimensions quasicrystals can often be seen as a stacking of overlapping clusters. This is helpful in those cases where approximants of almost the same composition exist. The clusters found are of various types. One may use these clusters to make a classification. The clusters are called Mackay, Bergman or Tsai clusters (Fig. 18). They occur in AlPdMn, AlLiCu and CdYb, respectively. A Mackay cluster contains 54 atoms, a Bergman cluster 137 and a Tsai cluster 92 atoms.

When one adopts the definition of quasicrystals that requires a symmetry that is not crystallographic in three dimensions, the superspace groups belong to the following classes: pentagonal, octagonal, decagonal, dodecagonal and icosahedral. The list of superspace groups for these classes was derived in Janssen (1988) and, later, in Rabson et al. (1991) and Mermin (1992). There is some freedom in the definition of equivalence. This determines the number of groups in the list. The most general definition agrees with that of space groups in $n$ dimensions: isomorphism or affine conjugation, which is the same according to Bieberbach $(1911,1912$,$) . Whether it makes$ sense to also include the type of affine conjugation is open to debate. In three dimensions there are 219 space groups according to the definition here, but 230 if the affine conjugation keeps the handedness invariant. For aperiodic crystals this issue has not yet been addressed.
Among the icosahedral quasicrystals the most important families are the following (Tsai, 2013):

(i) AlLiCu (Ball \& Lloyd, 1985; Dubost et al., 1986). Single grains with an icosahedral morphology could be grown, which made it possible to start studying physical properties. A first model was presented in de Boissieu et al. (1991).

(ii) $\mathrm{AlCuFe}$ (Tsai et al., 1987). The $\mathrm{i}-\mathrm{AlCuFe}$ quasicrystal is F-type. A model has been proposed in Katz \& Gratias (1995); see Fig. 22.

(iii) AlPdMn (Tsai et al., 1990). In the search for new stable quasicrystals the ela ratio played an important role (see Fig. 19). A composition was chosen to obtain a specific ela ratio. One of the results of the search on the basis of this quantity was AlMnPd, which was investigated extensively on its crystallographic and physical properties.

(iv) $\mathrm{ZnMgRE}(\mathrm{RE}=$ rare earth; Tsai et al., 1994). This family, with $\mathrm{RE}=\mathrm{Y}, \mathrm{Gd}, \mathrm{Tb}, \mathrm{Dy}$, Ho and Er, is also F-type. A

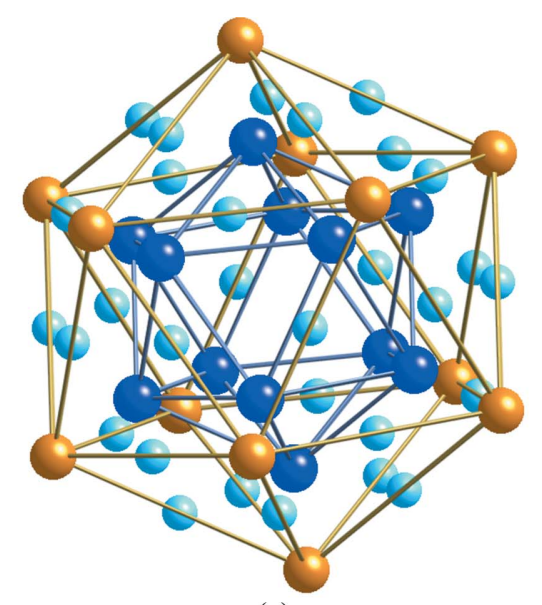

(a)

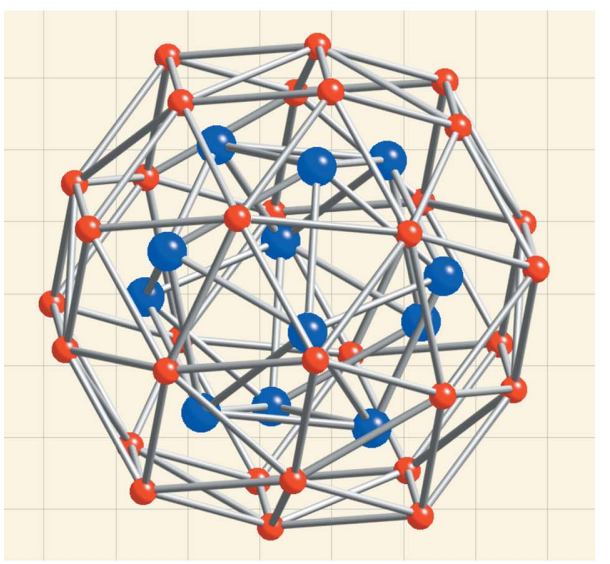

(b)

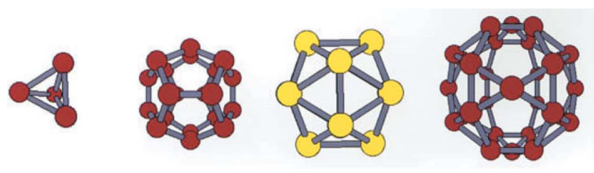

(c)

Figure 18

Three fundamental clusters: (a) Mackay cluster; (b) Bergman cluster; (c) Tsai cluster. 
P-type quasicrystal in this family is $\mathrm{ZnMgEr}$ (Sterzel et al., 2002).

(v) CdYb. The discovery of this compound was presented at the Aperiodic 2000 conference and published by Tsai et al. (2000). Owing to its relatively simple structure, and the fact that approximants are available, this was the first icosahedral quasicrystal for which the structure could be determined with quite high precision (Takakura et al., 2007).

4.4.6. The microscopic structure of quasicrystals. Although it was relatively simple to construct the lattice in superspace for quasicrystals, the microscopic structure remained obscure for a long time. The phrase 'Where are the atoms?' used by Per Bak (Bak, 1986) has often been quoted.

The first quasicrystal structure determination was for a decagonal phase (Steurer et al., 1993; Cervellino et al., 2002). The decagonal phases consist of a periodic array of puckered planes with decagonal symmetry. The periodicity along the axis is usually even, 2, 4, 6 or 8 . A model for $d-\mathrm{Al}_{72} \mathrm{Ni}_{20} \mathrm{Co}_{8}$ was published in Takakura et al. (2001).

Apart from the decagonal phases, quasicrystals with periodicity along one axis and octagonal or dodecagonal symmetry in the planes perpendicular to the axis have also been found. However, their number is much smaller; after the discovery of Shechtman, a paper appeared with a dodecagonal structure in $\mathrm{NiCr}$ (Ishimasa et al., 1985). Another example is the dodecagonal chalcogenide $\mathrm{Ta}_{16} \mathrm{Te}$ (Conrad et al., 1998). An example of an octagonal structure is $\mathrm{MnSiAl}$ (Wang et al., 1987). There are as yet no precise structure determinations for these systems.

For icosahedral quasicrystals (rank 6) several models were proposed and studied. The stability and formation of icosahedral quasicrystals was discussed in the framework of the Landau theory of phase transitions in Bak (1985a). The basis of that work was the consideration of the stability of alloys in
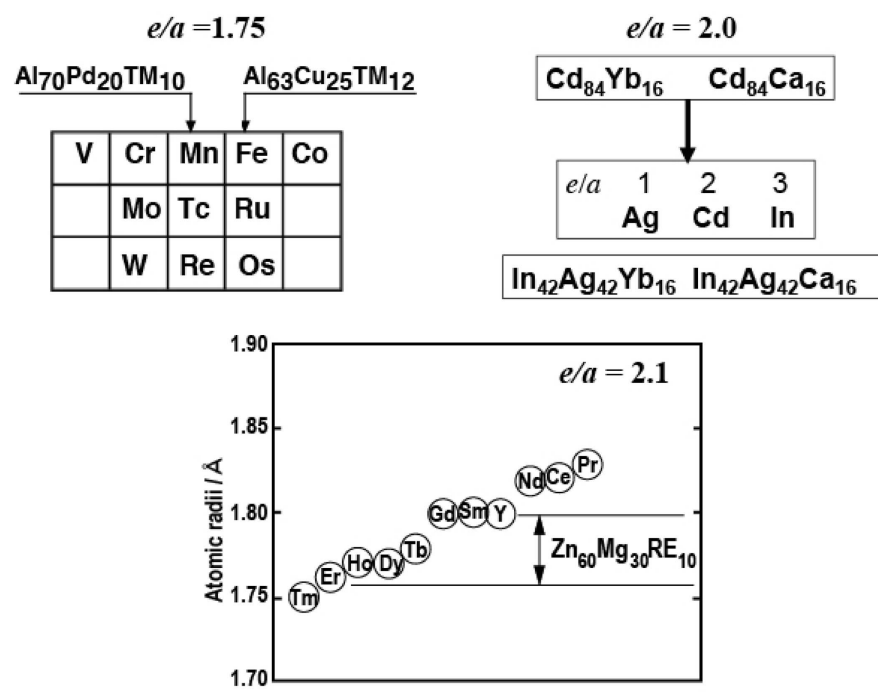

Figure 19

Three families of icosahedral quasicrystals. The $e / a$ ratio is an important factor in the formation of stable quasicrystals (reproduced from Tsai, 2013, with permission from The Royal Society of Chemistry).

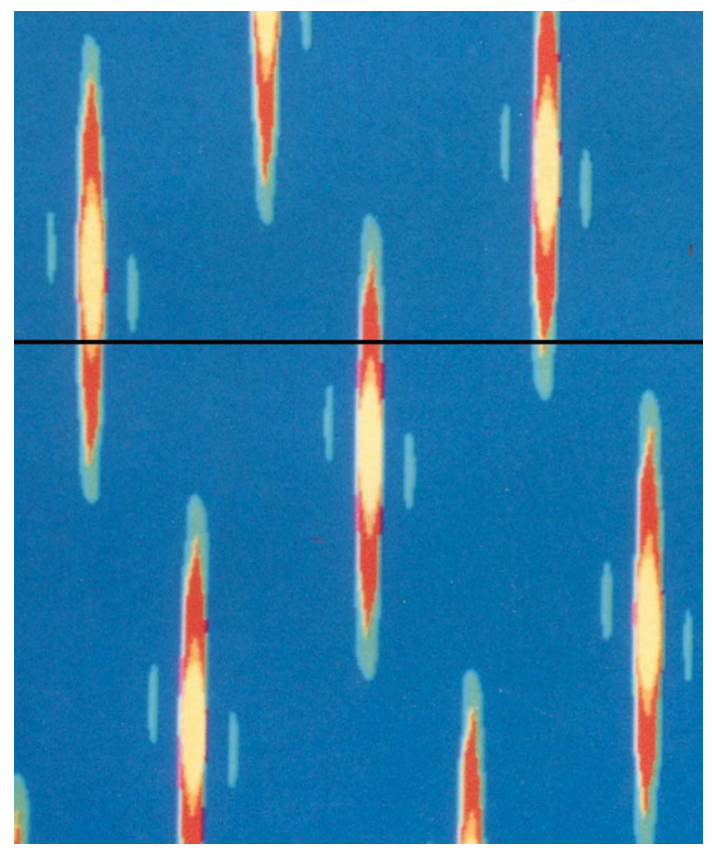

Figure 20

The two-dimensional Patterson function for the (one-dimensional) Fibonacci chain.

Alexander \& McTague (1978). An early attempt to model the structure was published by Guyot \& Audier (1985). This was based on three-dimensional icosahedral tiling, and the knowledge of diffraction patterns and chemical composition. The quality of the samples by that time was not sufficient to proceed further than a qualitative approach. In Elser \& Henley (1985) the correspondence between the crystalline $\alpha$ AlMnSi and the icosahedral phase was pointed out. A model based on a six-dimensional Patterson analysis was published

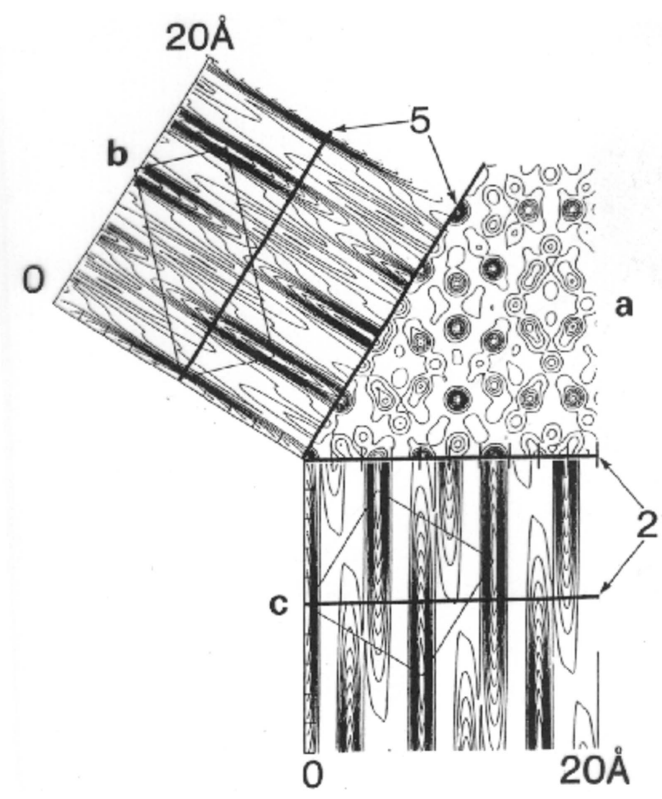

Figure 21

Two cuts through the Patterson function of an icosahedral quasicrystal (after Gratias et al., 1988). 

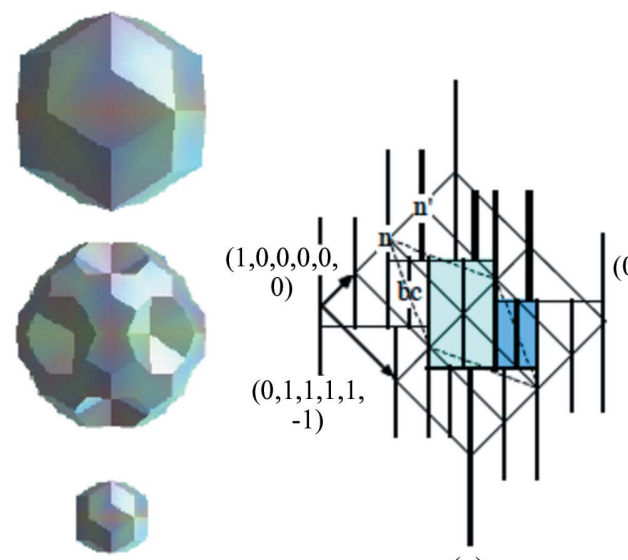

(a)

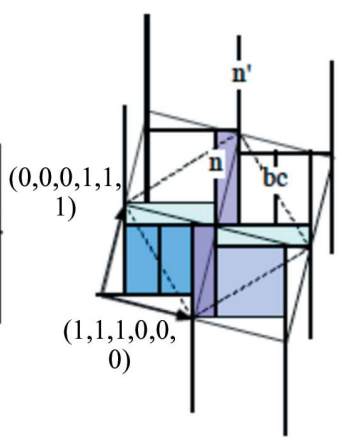

(b)

Figure 22

Atomic surfaces for an F-type icosahedral quasicrystal (after Gratias et al., 2000). Left: Three atomic surfaces, in 000000,100000 and $(\overline{1} 1111 \overline{1}) / 2 ;(a)-(c)$ four two-dimensional cuts along a fivefold, a threefold and two threefold axes (after Gratias et al., 2000).

by Boudard et al. (1992). However, the discrepancy with the experimental data was still quite large. Other models were proposed by Yamamoto \& Hiraga (1988), Katz \& Gratias (1995), Yamamoto et al. (2003) and by Quiquandon \& Gratias (2006).

A global idea of the structure of an icosahedral quasicrystal may be obtained from the Patterson function, the Fourier transform of the intensities of the diffraction spots

$$
P\left(\mathbf{r}, \mathbf{r}_{I}\right)=\sum_{\mathbf{H}}|F(\mathbf{H})|^{2} \exp \left(i\left(\mathbf{H} \cdot \mathbf{r}+H_{I} \cdot \mathbf{r}_{I}\right)\right) .
$$

An example is given for the Fibonacci chain in Fig. 20. The case of an icosahedral quasicrystal is clearly more difficult, because the Patterson function is a function of six variables. An idea of the atomic surfaces may be obtained by looking at two-dimensional cuts along a plane. For this plane one might

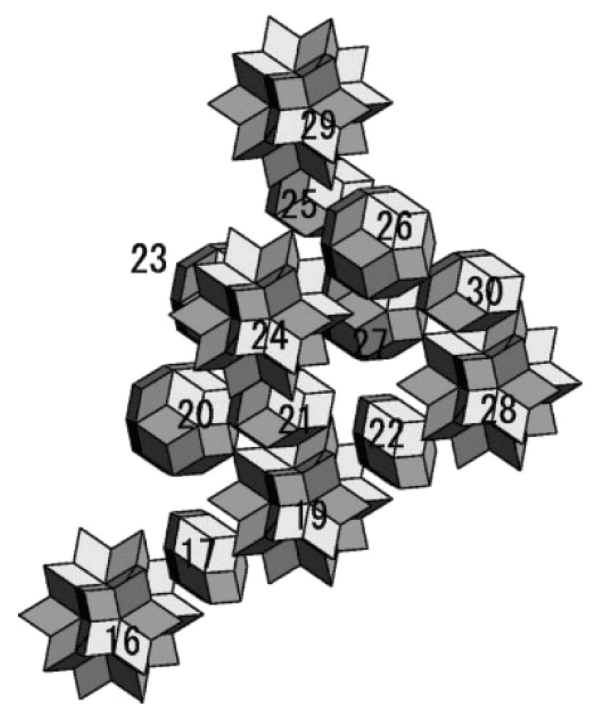

Figure 23

Exploded view of one of the three atomic surfaces (at 000000) for an Ftype icosahedral AlPdMn (after Yamamoto et al., 2003).<

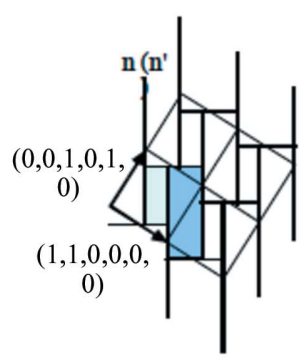

(c) choose a cut through two fivefold axes in the unit cell: $\left(\frac{1}{2} \frac{1}{2} \frac{1}{2} \frac{1}{2} \frac{1}{2} \frac{1}{2}\right)$ and $\left(\frac{1}{2} 00000\right)$. Such a section is given in Fig. 21.

It turns out that the atomic surfaces in icosahedral quasicrystals are often located at highsymmetry points in the six-dimensional unit cell. A first approximation then is to put spheres at carefully chosen positions in the unit cell, leaving the radius as an adjustable parameter. This is the spherical model.

This model does not satisfy the so-called closeness condition. This means the following. If one shifts the physical space in a direction in internal space, atoms disappear when the border of an atomic surface leaves the physical space. It should appear somewhere else, which means that the projection of a border of an atomic surface should coincide with the projection of a border of another atomic surface. This can be obtained when the projection of the borders of two atomic surfaces coincides with the plane that cuts the connection of the centra perpendicularly. In the model of Katz and Gratias this closeness condition has been satisfied (see Fig. 22) Instead of spheres, the atomic surfaces are assemblies of three-dimensional polytopes. The projection of the atomic surfaces on the physical space are points, which corresponds to the cut-and-project method for aperiodic tilings. In principle, a displacement field on the atomic surfaces in the direction of the physical space would give a modulated quasicrystal.

Another six-dimensional model, for face-centered icosahedral AlPdMn, was presented by Yamamoto et al. (2003). Also here the atomic surfaces are clusters of three-dimensional polytopes (see Fig. 23).

The models mentioned here gave satisfactory agreement, but a number of discrepancies were not solved. The structure determination of a quasicrystal improved later when binary quasicrystals were discovered (Guo et al., 2002), for which

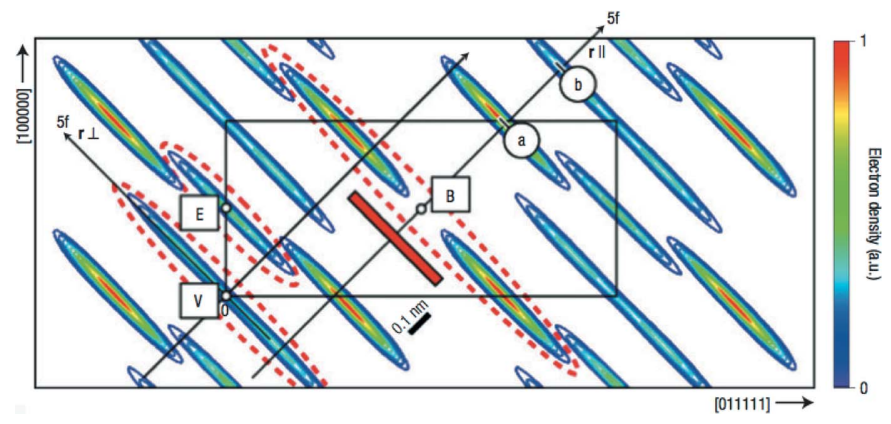

Figure 24

Structure of i-CdYb from a cut with a plane through two fivefold axes: 100000 and 011111 (Takakura et al., 2007). Reproduced with permission from Macmillan Publishers Ltd. Copyright 2007. 


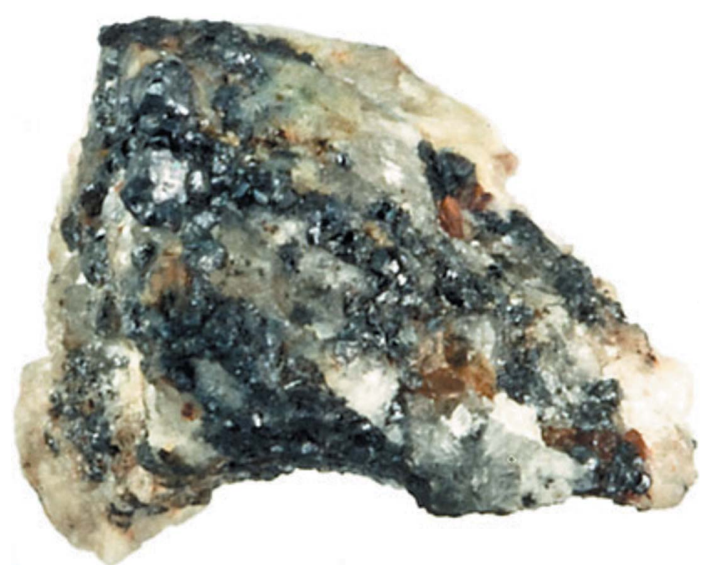

Figure 25

A quasicrystalline sample from the Museo di Storia Naturale dell'Università degli Studi di Firenze (Bindi et al., 2009). Reproduced with permission from AAAS.

approximants exist such that one could analyse the constituting clusters, which are in this case the Tsai clusters mentioned earlier. This allowed the construction of the atomic surfaces (Fig. 24). The result was published in Takakura et al. (2007).

4.4.7. Magnetic quasicrystals. Quasicrystals often contain atoms with magnetic moments. Nevertheless, it seems that quasi-crystallinity and magnetic structure are incompatible. The theoretical formalism to describe the magnetic symmetry is available. Magnetic quasicrystals could be described by magnetic superspace groups (Lifshitz, 1998; Janssen et al., 2007), but a magnetically ordered quasicrystal has not yet been found. On the other hand, such magnetic ordering has been found in approximants. Binary icosahedral quasicrystals with magnetic moments have been discussed by Goldman et al.

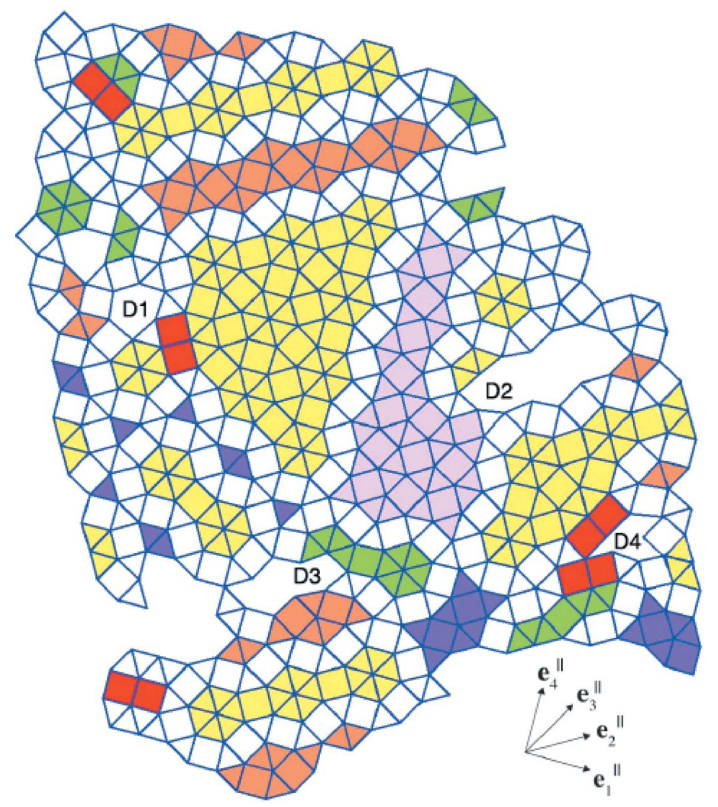

Figure 26

Tiling obtained from TEM pictures of a three-component polymer system, showing the dodecagonal structure (after Hayashida et al., 2007).
(2013). Their conclusion: 'Our results, so far, support the idea that the presence of aperiodic, rather than periodic, order disrupts or frustrates long-range magnetic order'.

4.4.8. Natural quasicrystals. There is an adagium saying that whatever is possible will be found in nature. In that sense it was not surprising that quasicrystals were found as minerals. After all, there are many examples of incommensurate modulated phases in minerals. Nevertheless, it was exciting when Luca Bindi discovered a quasicrystal in a sample from East Siberia in his geological collection. Later in that same region, other samples were found (Fig. 25). An interesting point is that the isotope distribution in the sample seems to deviate from the usual one, which led to speculation about a possible non-terrestrial origin of the sample (Bindi et al., 2009).

4.4.9. Mesoscopic quasicrystalline structures. Non-crystallographic symmetries can also be found in mesoscopic structures created artificially by the atomic optical fabrication of structures. Using interference from laser beams the deposition of atoms may be manipulated to form sub-wavelength quasi-periodic structures. Five laser beams in a plane with angles of $72^{\circ}$ create a Cr structure with fivefold symmetry (Jurdik et al., 2004).

Besides these artificially made mesoscopic structures, the spontaneous formation of systems with non-crystallographic symmetry also occurs. Liquid crystals discussed in $\$ 4.3$ showed incommensurability in one direction. For some systems consisting of dendrimers (tree-shaped molecules) a sequence of phase transitions was found, where one of the phases shows dodecagonal symmetry (Zeng et al., 2004; Ungar \& Zeng, 2005). It is periodic in one direction and dodecagonal in the perpendicular directions. The periodicity is very close to

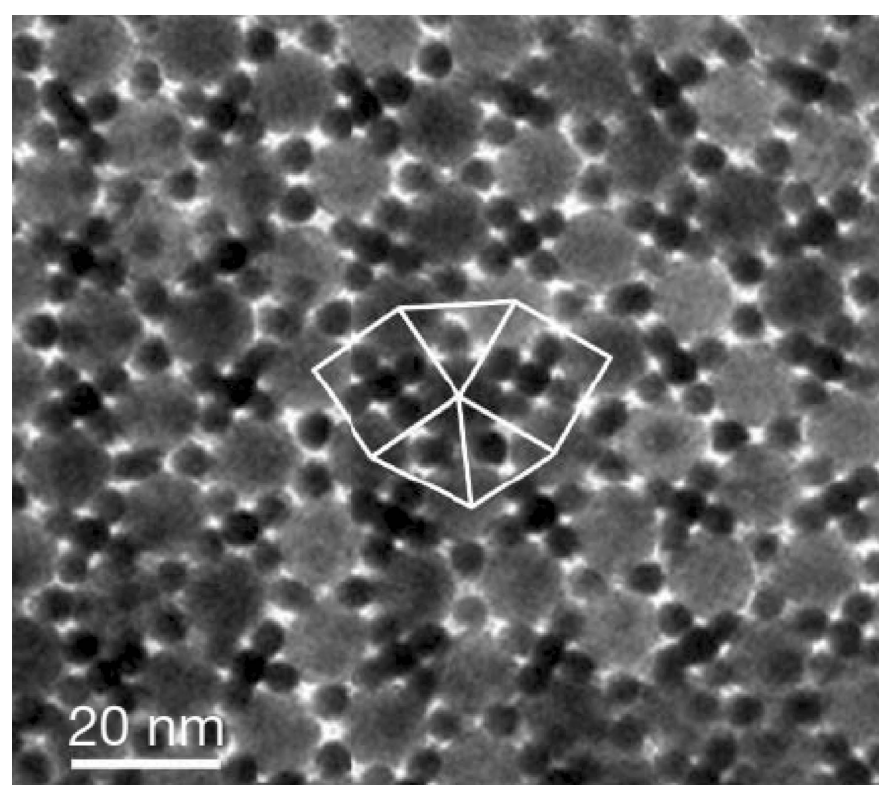

Figure 27

TEM pictures of a two-component system of nanoparticles (after Talapin et al. 2009). Reproduced with permission from Macmillan Publishers Ltd. Copyright 2009. 
distances in the other phases, with space groups $P m \overline{3} n$ and $P 4_{2} / m n m$. These are Frank-Kasper phases of tetrahedrally closed-packed structures.

Another system where dodecagonal symmetry has been observed is a three-component polymer system (Hayashida $e t$ al., 2007). Transmission electron microscopy shows a dodecagonal tiling with triangles and squares (Fig. 26).

The dodecagonal symmetry can be found on several length scales. It also occurs in assemblies of nano-particles. An example is presented in Talapin et al. (2009). The system consists of $13.4 \mathrm{~nm} \mathrm{Fe}{ }_{2} \mathrm{O}_{3}$ and $5 \mathrm{~nm} \mathrm{Au}$ particles. Depending on the relative concentration of the constituting components, there is a phase with dodecagonal symmetry as shown in the diffraction pattern. A TEM picture is presented in Fig. 27.

Not only the scale of these mesoscopic structures is different from that of the quasicrystalline metallic alloys, but also the coherence length is different. This means that just as liquid crystals are not crystals, not all mesoscopic systems with 'forbidden' symmetries are quasicrystals. The fact that they show unusual symmetry is, however, very interesting.

\subsection{General superspace approach to aperiodic crystals}

Presented here is a summary of the general construction rules for aperiodic crystals, as introduced in the preceding sections for the various classes of aperiodic crystals (Fig. 28). For clarity, we sometimes repeat what has been said before. For all aperiodic crystals, the (sharp) diffraction spots can be indexed as

$$
\mathbf{H}=\sum_{i=1}^{n} h_{i} \mathbf{a}_{i}^{*},
$$

where $n$ is larger than the dimension of physical space. These spots then are projections of reciprocal lattice vectors in $n$ dimensions.

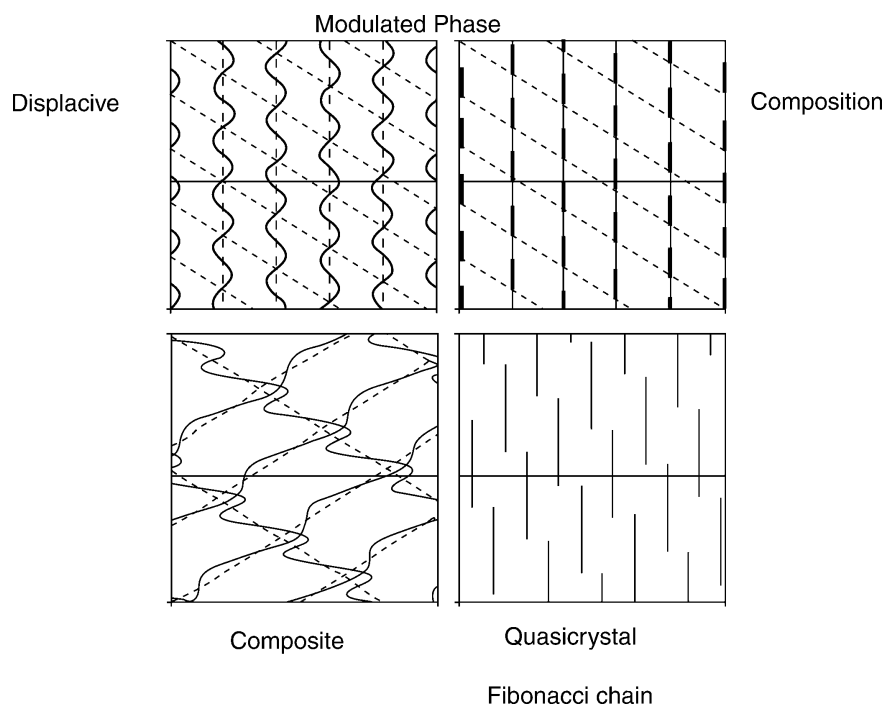

Figure 28

Embedding in superspace for four different classes of aperiodic crystals: Displacive and occupational modulation, incommensurate composite, quasicrystal (i.e. a Fibonacci chain).

$$
\left(\mathbf{H}, \mathbf{H}_{\mathrm{I}}\right)=\sum_{i=1}^{n} h_{i}\left(\mathbf{a}_{i}^{*}, \mathbf{b}_{i}^{*}\right)
$$

The diffraction pattern is invariant under a (finite) point group $\mathbf{K}$, which may or may not be a crystallographic point group in three dimensions. The invariance means that

$$
R \mathbf{a}_{i}^{*}=\sum_{j=1}^{n} \Gamma^{*}(R)_{j i} \mathbf{a}_{j}^{*} .
$$

Here the matrix elements $\Gamma^{*}(R)_{j i}$ are integers. The matrices form a finite group of $n \times n$ matrices $\Gamma^{*}(K)$. Because the group is finite, it is equivalent to a group of orthogonal matrices

$$
\Gamma^{*}(R) \sim\left(\begin{array}{cc}
R_{\mathrm{E}} & 0 \\
0 & R_{\mathrm{I}}
\end{array}\right)
$$

The group of matrices for the action in direct space is given by $\Gamma(K)$ with $\Gamma(R)_{i j}=\Gamma^{*}\left(R^{-1}\right)_{j i}$. From the point group $\Gamma(K)$ followed the metric relations for the embedded lattice, i.e. the direct lattice corresponding to the reciprocal lattice on the basis $\left(\mathbf{a}_{i}^{*}, \mathbf{b}_{i}^{*}\right)$. The possible metric tensors, with elements $g_{i j}=\mathbf{a}_{i} \cdot \mathbf{a}_{j}+\mathbf{b}_{i} \cdot \mathbf{b}_{j}$, follow from

$$
\Gamma(R) \cdot g \cdot \Gamma(R)^{\mathrm{T}}=g .
$$

This means that the number of free parameters in this metric tensor is fixed by these relations. Using a group-theoretical formulation, one may say that the number of free parameters in $g$ is the number of times the identity representation occurs in the decomposition of the symmetrized square of the vector representation, i.e. the representation given by the matrices $\Gamma(R)$.

The embedded density

$$
\rho\left(\mathbf{r}, \mathbf{r}_{\mathrm{I}}\right)=\sum_{\mathbf{H}} \hat{\rho}(\mathbf{H}) \exp \left(i \mathbf{H} \cdot \mathbf{r}+i \mathbf{H}_{\mathrm{I}} \cdot \mathbf{r}_{\mathrm{I}}\right)
$$

is invariant under an $n$-dimensional superspace group with elements $g=\left\{\left(R_{\mathrm{E}}, R_{\mathrm{I}}\right) \mid\left(\mathbf{t}_{\mathrm{E}}, \mathbf{t}_{\mathrm{I}}\right)\right\}$. Owing to the phase problem, the point group consisting of the elements $R_{\mathrm{E}}$ in this superspace group may be a subgroup of the elements $R$ in equation (36).

Knowing the point-group matrices $\Gamma(R)$, the superspace groups may be calculated using algorithms given in Zassenhaus (1948) and Janssen et al. (1969). A listing requires the definition of the equivalence relation adopted. Although not always mentioned by the authors of superspace group listings, some use isomorphism as the equivalence relation. This is the same as the affine conjugate: two superspace groups are equivalent if there is an affine transformation (an invertible linear transformation combined with a translation) mapping one onto the other. This criterion gives 219 space groups in three dimensions. For incommensurate modulated crystal phases a stricter relation is used. The linear transformation should leave the lattice of the main reflections invariant. One may write the superspace point group elements as in equation (15). Then the linear transformation in the connecting affine transformation should be of the form 


$$
S=\left(\begin{array}{cc}
S_{\mathrm{E}} & 0 \\
S_{M} & S_{\mathrm{I}}
\end{array}\right)
$$

The possibility of the requirement that either $S, S_{\mathrm{E}}$ or $S_{\mathrm{I}}$ should have a positive determinant (in three dimensions there are 230 space groups if $S$ is required to have a positive determinant) has barely been considered. The fact that the equivalence relation for superspace groups for modulated phases is stricter is one of the reasons why the lists of superspace groups and of conventional higher-dimensional space groups differ. The other reason is that for modulated phases the point group is a crystallographic (magnetic) group in three dimensions. Therefore, the list given in Vol. C of the International Tables for Crystallography differs from that given in Brown et al. (1978). The latter considers isomorphism as the equivalence principle, but on the other hand allows point groups that are not crystallographic in three dimensions (e.g. with fivefold rotations).

Modulated phases have a basic structure with space-group symmetry. For the notation this space group is used as a first component. A second component gives information about the basic satellites, with the rule that basic satellites connected by a point group element are indicated by one symbol. The third component gives the translations in internal space associated with the elements indicated by symbols in the basic spacegroup symbol. An example is the superspace group for $\gamma$ $\mathrm{Na}_{2} \mathrm{CO}_{3}$. The basic space group is the monoclinic space group $P 2 / m$. The basic satellite, $\mathbf{q}$, is at $\alpha \mathbf{a}^{*}+\gamma \mathbf{c}^{*}$. Since $\mathbf{q}$ is invariant under the mirror and is inverted under the rotation, the superspace point group is $2 / m(\overline{1} 1) P$. The superspace group has the translation $\frac{1}{2} \mathbf{e}_{4}$ in internal space associated with the rotation, and is zero with the mirror. Therefore, the symbol of the superspace group is $P 2 / m(\alpha 0 \gamma) s 0$. Here $s$ means the component $\frac{1}{2} \mathbf{e}_{4}$ (in internal space) of the non-primitive translation associated with $2(\overline{1})$.

For incommensurate composites one may usually distinguish a host system, which has the structure of a modulated phase. Then the symbol for modulated phases can be used, but it should be noted that two such symbols could refer to isomorphic groups, even to groups that should be considered as equivalent according to $\$ 4.2$. An alternative is the use of symbols distinguishing isomorphism classes, as will be discussed for quasicrystals. An example is $\mathrm{TTF}_{7} \mathrm{I}_{5-x}$. The TTF host structure has a basic structure with the space group $C 2 / \mathrm{m}$. The spots not belonging to the main reflections of the TTF structure can be considered as satellites. Then the superspace group can be denoted by $C 2 / m(\alpha 1 \gamma) 0 s$ (again the $s$ denotes a non-primitive translation $\frac{1}{2} \mathbf{e}_{4}$ associated with the mirror $m$ ). On the other hand, the notation for four-dimensional space groups can be used, giving the symbol $C 2 / m_{d}(\overline{1} 1)$. This means that there is a centering in direct space $\left(\frac{1}{2} \frac{1}{2} 0\right)$, a four-dimensional point group $2 / m(\overline{1} 1)$ and a non-primitive translation $\frac{1}{2} \mathbf{e}_{4}$ for the element $(\mathrm{m}, 1)$. The use of a symbol like that for modulated phases means that the three-dimensional point group is necessarily crystallographic. Therefore, the second choice would be the only one for the fictitious composite previously mentioned, with two subsystems, each with tetra- gonal basic symmetry, rotated over $\pi / 4$ with respect to each other. Here the point-group symmetry of the diffraction pattern is octagonal, which cannot be the symmetry of one of the two subsystems. Then the notation for the superspace group is $P 8_{4} / \mathrm{mmm}\left(8^{3} / \mathrm{mmm}\right)$.

For quasicrystals the symbols adopted for modulated phases are not appropriate. Here one can use the symbols for space groups in higher-dimensional spaces, as proposed by a Nomenclature Committee of the IUCr (Janssen et al., 2002). A symmorphic P-type icosahedral quasicrystal has a superspace group that is denoted by $P 5 \overline{3} m\left(5^{2} \overline{3} m\right)$. There are, for this symmorphic group, no symbols for non-primitive translations. Generators of the point group are $5\left(5^{2}\right), \overline{3}(\overline{3})$ and $m(m)$. On the basis given in equation (32), the three generators are given by

$$
\begin{aligned}
5\left(5^{2}\right) & =\left(\begin{array}{llllll}
1 & 0 & 0 & 0 & 0 & 0 \\
0 & 0 & 1 & 0 & 0 & 0 \\
0 & 0 & 0 & 1 & 0 & 0 \\
0 & 0 & 0 & 0 & 1 & 0 \\
0 & 0 & 0 & 0 & 0 & 1 \\
0 & 1 & 0 & 0 & 0 & 0
\end{array}\right), \\
3(3) & =\left(\begin{array}{cccccc}
0 & 0 & 0 & 0 & 0 & 1 \\
1 & 0 & 0 & 0 & 0 & 0 \\
0 & 0 & 0 & 0 & 1 & 0 \\
0 & 0 & -1 & 0 & 0 & 0 \\
0 & 0 & 0 & -1 & 0 & 0 \\
0 & 1 & 0 & 0 & 0 & 0
\end{array}\right) .
\end{aligned}
$$

and the inversion in six dimensions. The first generator has a two-dimensional invariant space, generated by (100000) and (011111). The second generator also has a two-dimensional invariant space, spanned by (110001) and (001110). It is clear that there is a two-dimensional invariant space because the first generator has irreducible components (over the real numbers) $5,5^{2}, 1$ and 1 , and similarly for the second generator.

As the diffraction spots in physical space are the projections of reciprocal lattice vectors in superspace [i.e. $F(\mathbf{H})=$ $\left.F\left(\mathbf{H}, \mathbf{H}_{\mathrm{I}}\right)\right]$, the structure factor in physical space may be calculated as that of the lattice periodic structure in superspace. The main difference with the normal procedure in three dimensions is that there the atoms are specified by an atomic scattering factor and a position in space, whereas in superspace the atomic position is extended to an atomic surface. Therefore, equation (21) generalizes to

$$
F(\mathbf{H})=\sum_{j} \int_{\Omega_{j}} f\left(j, \mathbf{r}_{\mathrm{I}}\right) \exp \left[i \mathbf{H} \cdot \mathbf{r}\left(\mathbf{r}_{\mathrm{I}}\right)+i \mathbf{H}_{\mathrm{I}} \cdot \mathbf{r}_{\mathrm{I}}\right] \mathrm{d} \mathbf{r}_{\mathrm{I}} .
$$

Here the integration is over the atomic surfaces, $\Omega_{j}$, in the unit cell, with integration parameter $\mathbf{r}_{\mathrm{I}}$. The atomic scattering function and the Debye-Waller factor are denoted by $f\left(j, \mathbf{r}_{j}\right)$. A special case of this expression is that for incommensurate modulated crystal phases. Another case is that of quasicrystals with atomic surfaces parallel to $V_{\mathrm{I}}$. Then the section method is equivalent to the cut-and-project method and $\mathbf{r}\left(\mathbf{r}_{\mathrm{I}}\right)$ does not depend on $\mathbf{r}_{\mathrm{I}}$. 
The diffraction peaks in physical space correspond to the lattice points of the reciprocal lattice. Owing to the difference in their origin, the distribution of intensities is different for the different classes. For modulated phases, the main reflections are usually the stronger ones. Their relative intensities are determined by the arrangement in the unit cell. The satellites are usually weaker, and higher-order satellites are often too weak to be observed. For incommensurate composites, there are two or more subsystems each with a set of main reflections, because every subsystem is a modulated structure. Therefore, the main reflections of each subsystem are expected to be strong, but summation peaks (at positions that are the sum of main reflections of different subsystems) are usually weak. For quasicrystals, the diffraction peaks are mainly determined by integrals over the atomic surfaces, which are, in the first approximation, parallel to the internal space. They will fall off for larger internal space components of the reciprocal lattice vectors (Fig. 29).

For a lattice periodic function the Fourier transform, and also the structure factor, consists of sharp Bragg peaks on the nodes of the reciprocal lattice. In mathematical terms, this is a point spectrum. If disorder is present, there is another contribution to the scattering, the diffuse scattering. This corresponds to a second component, the absolute continuous spectrum. For general systems, there may be an additional component, the singular continuous spectrum. For some tilings, it can be shown that such a singular continuous component exists. There are some mathematical statements about the scattering, but the question which systems will give a point spectrum ('which systems diffract?') is not yet answered.
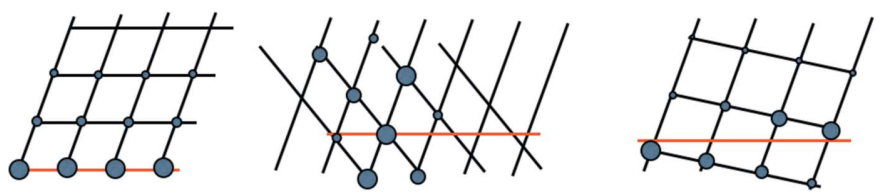

Figure 29

Intensity of the diffraction peaks are those of the peaks on the reciprocal lattice points in superspace. These are illustrated for incommensurate modulated structures, composites and quasicrystals, respectively.
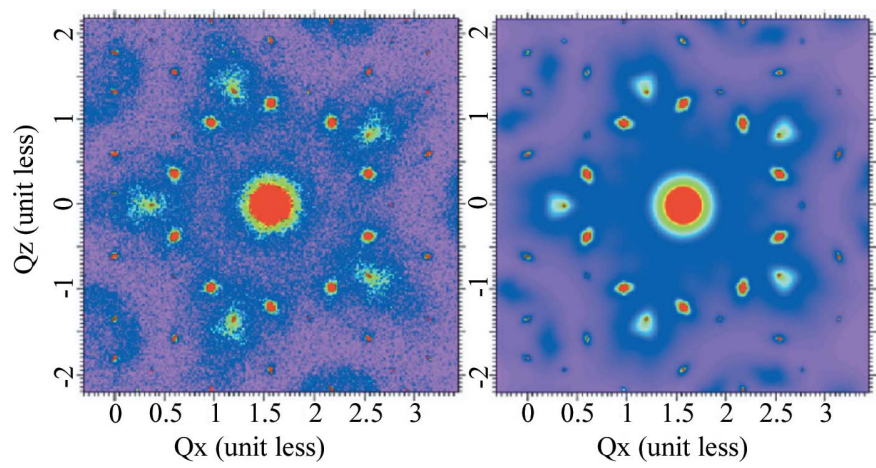

Figure 30

Phason diffuse scattering, observed and calculated using the elastic constants for icosahedral quasicrystals (reproduced from de Boissieu, 2012, with permission from The Royal Society of Chemistry).
Diffuse scattering occurs in both lattice periodic and aperiodic crystals. There is an additional contribution for aperiodic crystals. When an aperiodic crystal is perturbed and the perturbation can be described as a displacement of the atomic surfaces in internal space, this is called a phason (see §5.4). In quasicrystals such phason diffuse scattering has been observed (see Fig. 30). It can be explained using elasticity theory for quasicrystals (\$5.4).

The structure factor, as given in equation (42), is an approximation which neglects multiple scattering. This is not correct for electron diffraction. However, the dynamical scattering theory for aperiodic crystals has not yet been developed.

\subsection{Alternative descriptions of aperiodic crystals}

Several phase transitions towards an incommensurate modulated phase occur because of the softening of a vibration mode (see Fig. 31). The frequency of this mode becomes zero at the phase transition temperature. The mode may be characterized by means of irreducible representations of the space group of the parent phase, as used in Landau theory. Especially for displacive modulations this gives an alternative for the description via superspace. There is, however, a very close relationship of the two methods. We illustrate this point for a modulation with one wavevector, $\mathbf{q}$, such that the irreducible representation of the group of $\mathbf{q}$, denoted by $\mathrm{K}_{\mathbf{q}}$, is onedimensional. The generalization to more points of the star of $\mathbf{q}$ and for higher-dimensional representations is straightforward, but a little complicated (Janssen \& Janner, 1984). The representation of the space group is characterized by the vector $\mathbf{q}$ and the irreducible representation of $\mathrm{K}_{\mathbf{q}}$. The latter is characterized by the character $\chi(R)=\exp (i \phi(R))$, where $g=\{R \mid \mathbf{a}\}$ is an element of the space group. The action of $g$ on the displacement wave then gives a phase shift factor equal to

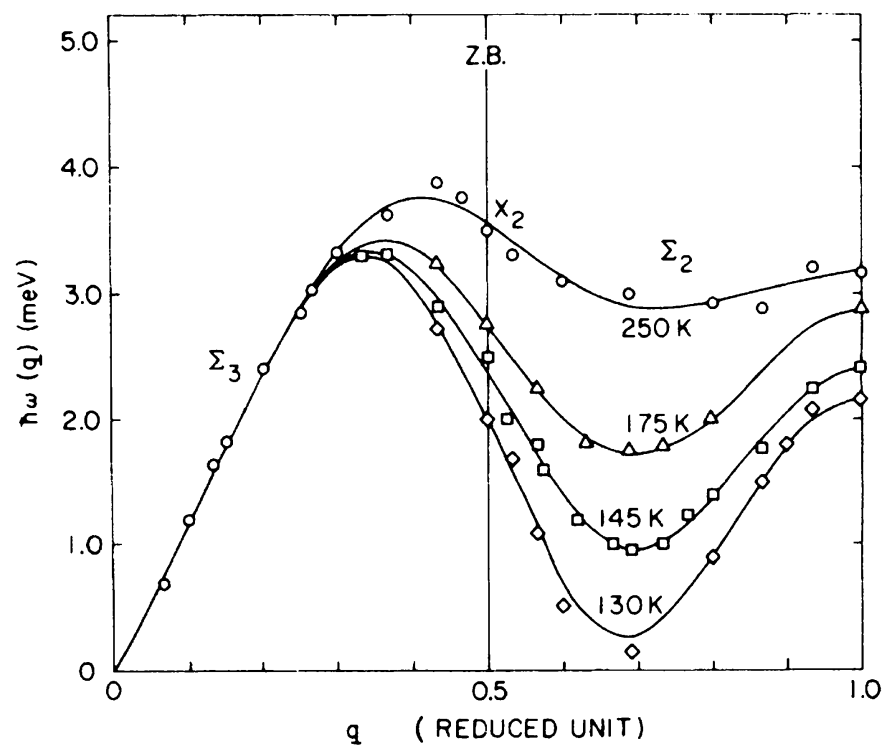

Figure 31

Soft mode in $\mathrm{K}_{2} \mathrm{SeO}_{4}$ leading to an incommensurate phase transition (Iizumi et al., 1977). 
$\exp (i \mathbf{q} \cdot \mathbf{a}) \chi(R)$. The $\chi(R)$ factor is just a shift of the phase of the mode, which is the same as the shift in internal space, when one considers the displacement as a function of the phase of the displacement wave. The description in terms of representation theory certainly in the beginning had a preference for those who worked on neutron scattering or Landau theory. The close relation between the two methods was also shown in Perez-Mato et al. (1984), which appeared shortly after Janssen \& Janner (1984).

The description of incommensurate composites was extended by van Smaalen (1991). In particular, the relation between the superspace group of the composite and the superspace groups of the subsystems, which are modulated phases, was analysed.

The description of quasicrystals in terms of lattice periodic structures in superspace is called the section method. Another, but related, method is that used for the description of aperiodic tilings, where the atoms are considered as projections of points in a strip parallel to the physical space. This method is the cut-and-project method. It was used for tilings and, for quasicrystals, in Duneau \& Katz (1985) and Kalugin et al. (1985a).

Bienenstock \& Ewald (1962) formulated the symmetry of lattice periodic crystals in reciprocal space. Because the Fourier components of a crystal are complex functions, one may combine the action of a space group element on the Fourier components with additional transformations of the phase of these components. The additional transformation is actually a gauge transformation as discussed in §3.2. Mermin and collaborators used this language for the description of quasicrystals. They introduce the concept of physical indistinguishability (Rokhsar et al., 1988). Then they show that the additional phase shifts of the Fourier components are linear functions of the wavevector. This then leads to an algorithm to calculate the symmetry groups (Rabson et al., 1991; Mermin, 1992). Certainly this means remaining in three dimensions and avoids a higher-dimensional formulation. The method is equivalent with the superspace approach, as can be seen from equation (25). Moreover, long-range order is most apparent in Fourier space, but the local structure is better seen in direct space. Therefore, the claim that the Bienenstock-Ewald method is much better suited for quasicrystals is a bit exaggerated. In software for structure determination the two dual spaces (direct and reciprocal) are used.

\subsection{Structure determination}

The problem of the structure determination of an aperiodic crystal can be translated into a problem of structure determination of a periodic structure in higher dimensions. There is, however, still a fundamental difference with the usual threedimensional crystals. There the number of degrees of freedom is three times the number of (point) atoms in the unit cell. For periodic structures in superspace there are extended atomic surfaces, and the number of degrees of freedom becomes infinite. Of course, the number of parameters to determine is also infinite when determining the charge distribution in a three-dimensional crystal.

For the structure determination of incommensurate modulated phases, and recently of incommensurate magnetic structures as well, methods and software have been developed. The first problem is the determination of the basic structure. This uses the extinction conditions for the main reflections. The basic structure has a relation to the average structure, which is the Fourier transform of the $\mathrm{F}(\mathbf{H})$ for the main reflections. Next to that, one has to parametrize the modulation functions, which may be a combination of a small number of Fourier components or may be discontinuous. In the latter case, piece-wise constant functions, also called Crenel functions, may be used. The programs REMOS by Yamamoto (2011) and JANA by Petřiček et al. (2006) have steadily been improved and applied to many materials. The use of these programs has become standard for incommensurate modulated phases and composites. They can be found via http:// jana.fzu.cz/ and http://wcp-ap.eng.hokudai.ac.jp/yamamoto/ spgr.html. The latter also offers software for the analysis of quasicrystals. A site with software for modulated structures is found in Chapuis (2013).

Besides the software, lists of superspace groups may also be found on the internet. They include the web sites of Yamamoto (http://www.ccp14.ac.uk/ccp/web-mirrors/remos/ yamamoto/table3/cubic.html), the EPFL (http://superspace.epfl.ch) and Stokes, Campbell and van Smaalen (http://stokes.byu.edu/ iso/ssg.php). Owing to the rapid increase in numbers with a rank higher than four, it is not feasible to publish them in a paper volume. That was still possible for rank four, where the list was published (together with an introduction to the theory and to the notation) in Vol. C of the International Tables for Crystallography (Janssen et al., 1992). Information about incommensurate phases and composites is available on the Bilbao Crystallographic Server (http://www.cryst.ehu.es), and general information about aperiodic crystals on the website of the Special Interest Group on Aperiodic Crystals (1998) of the ECA (http://sig3.ecanews.org).

For incommensurate composites the systematization of structure determination has not yet been achieved, but the structure has been determined for several composites. The work by van Smaalen should be mentioned here (van Smaalen, 2007).

For quasicrystals the situation is much more difficult. Here the number, position and shape of the atomic surfaces has to be determined. If there is chemical ordering on the atomic surfaces, or when the quasicrystals are modulated, the problem is even harder. There are few structure determination results, actually only for a couple of decagonal phases (Takakura et al., 2001; Cervellino et al., 2002) and for the icosahedral CdYb quasicrystal (Takakura et al., 2007).

A first insight into the structure of an aperiodic crystal may be obtained using the Patterson function (Cahn et al., 1988; Gratias et al., 1988). The positions of atoms in modulated phases and composites may first be parametrized, after which the parameters may be found using the expression for the structure factor [equation (42)]. 
A number of techniques have been developed to help structure determination. Here we may mention the contrastvariation method (Dubois \& Janot, 1987) and the direct method adapted to aperiodic crystals (Hao et al., 1987). In recent years a number of additional techniques have been developed, such as the maximum-entropy method (Haibach \& Steurer, 1996; Weber \& Yamamoto, 1997; van Smaalen et al., 2003) and the charge-flipping method (Oszlányi \& Sütő, 2004; Palatinus, 2013).

\subsection{Phase transitions}

Often, aperiodic crystal phases only occur in a certain temperature or pressure range. This implies that phase transitions are also interesting phenomena for aperiodic crystals.

For modulated crystals, the incommensurate phase is usually intermediate (Fig. 4). In many cases the hightemperature transition is caused by a soft mode, and the lower transition, where the irrational component(s) of the modulation wavevector becomes rational (a lock-in transition), is usually first order. Sometimes, there is only a partial lock-in, where one of the irrational components becomes rational. Then, generally, the rank of the aperiodic structure changes. An example is biphenyl. There are cases, e.g. BCCD, where a series of transitions appears with changing wavevector.

For composites, one subsystem may often be considered as the host, and the other as the guest(s). The latter occupies voids in the former structure. At high temperature the guest may be disordered and liquid-like, whereas for lower temperature the guest freezes which leads to the aperiodic crystal structure. This melting-freezing transition has often been observed. Apart from this transition, transitions between aperiodic crystal phases also occur. An example is nonadecane-urea, where the $p-T$ (pressure-temperature) phase

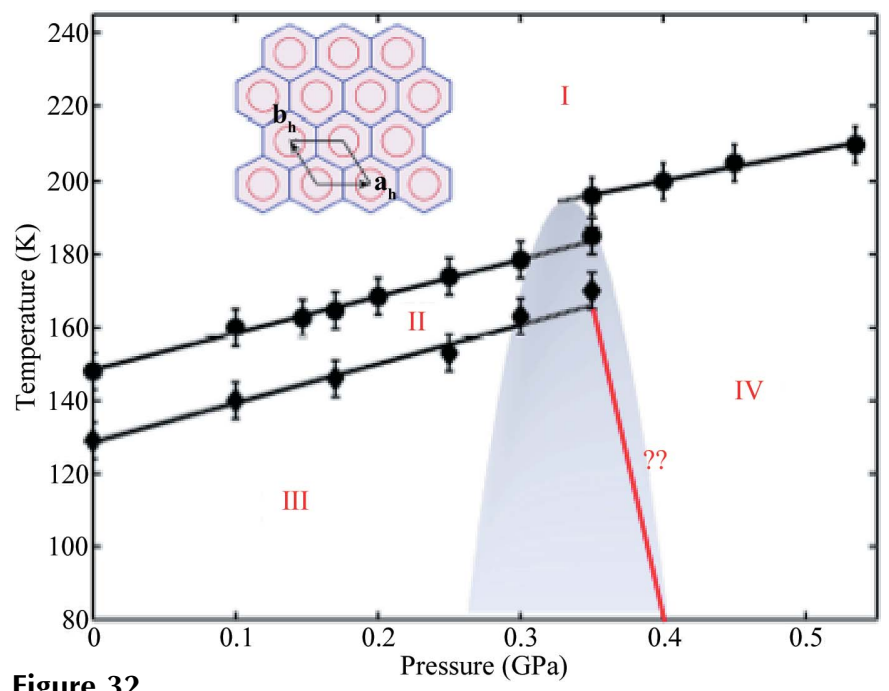

Figure 32

Temperature-pressure phase diagram of the fully deuterated nonadecane-urea. Phase I is hexagonal, phases II-IV orthorhombic. Phases I-IV have rank 4, 4, 5 and 5, respectively (Toudic et al., 2011, reproduced with permission from the European Physical Society). diagram shows at least four different phases (Fig. 32), of rank 4 or 5.

For quasicrystals, the situation is less clear. There are indications that a quasicrystal may become unstable at lower temperature, where a transition to an approximant structure could occur. Experiments here are difficult because of the slow kinetics.

An overview of the types of phase transitions in aperiodic crystals is given in Janssen (2014).

\subsection{Approximants, related materials}

Usually the modulation wavevector of an incommensurate phase changes with temperature. When this happens continuously for a one-dimensional modulation $(n=3+1)$, the value also reaches commensurate values. If this happens, the structure is lattice periodic and the symmetry group is a three-dimensional space group. These space groups may depend on the rational value and on the phase of the commensurate modulation. In this sense, the superspace group gives a unifying view of a whole family of structures. The structure for rational values of the modulation wave vector is called an approximant.

Approximants also appear for tilings and quasicrystals, at least in theory. Consider, as an example, the AmmannBeenker tiling. The four basis vectors of the Fourier module are $a(1,0), \frac{1}{2} a(\sqrt{2}, \sqrt{2}), a(0,1)$ and $\frac{1}{2} a(-\sqrt{2}, \sqrt{2})$. When one approximates $\frac{1}{2} \sqrt{2}$ by the rational number $L / N$, the resulting Fourier module is of rank two and the structure is lattice periodic. One can do this in a systematic way, for example, using the continued fraction expansion. For $\frac{1}{2} \sqrt{2}$ this series starts with $1,2 / 3,5 / 7,12 / 17,29 / 41$ and so on. A lattice periodic structure is obtained when the irrational number in the

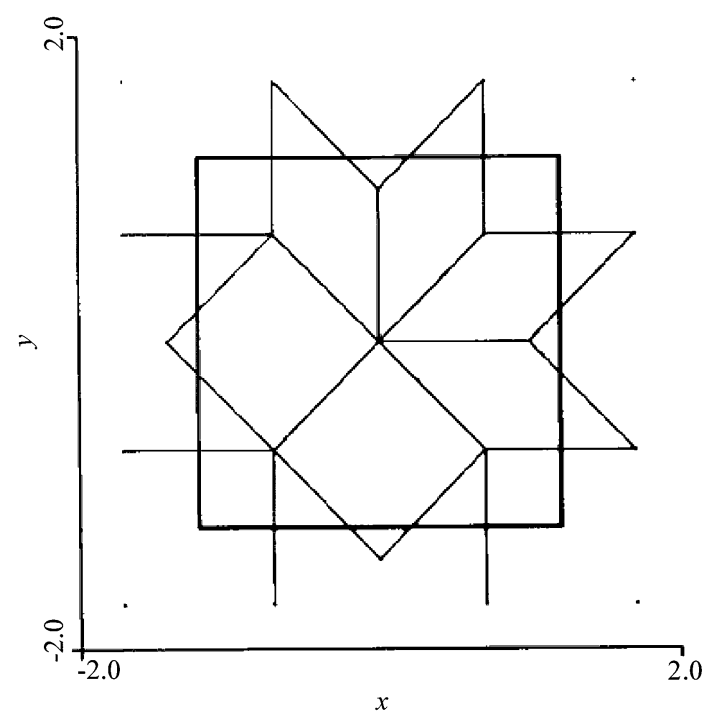

Figure 33

An approximant for the Ammann-Beenker tiling. The tiles (a square and a rhomb) are the same in both the aperiodic and the periodic tiling. The large square is a unit cell of the approximant. The two small squares and four rhombs also form together a unit cell, and the pattern of these six tiles is repeated. The approximant has, of course, not an eightfold symmetry. 
internal component of the basis vectors is replaced by the rational number $L / N$. In this case the tiles in physical space remain the same, but the distribution changes in such a way that the pattern has lattice periodicity (see Fig. 33).

For quasicrystals, the existence of approximants for alloys of almost the same composition as that for the quasicrystals has been a great help to understand the structure. For an approximant, standard structure determination gives the positions of the atoms, from which the clusters may be derived. If one assumes that the clusters in the approximants are similar to those in the quasicrystal, this gives a good starting point for the structure determination. For quasicrystals without approximant, this is much more difficult.

\subsection{Scaling transformations}

The space-time transformation given by equation (2) has two two-dimensional invariant subspaces. In one subspace the effect is an expansion with a factor $2+\sqrt{3}$ and in the other a contraction with a factor $2-\sqrt{3}$. Such non-Euclidean transformations occur also for aperiodic crystals, in particular for tilings (such as the Penrose tiling) and quasicrystals. The point group of the superspace group of the Penrose tiling is a group of $4 \times 4$ matrices. As a representation of the abstract point group, these matrices may be split into irreducible representations, one in physical space, one in internal space. A

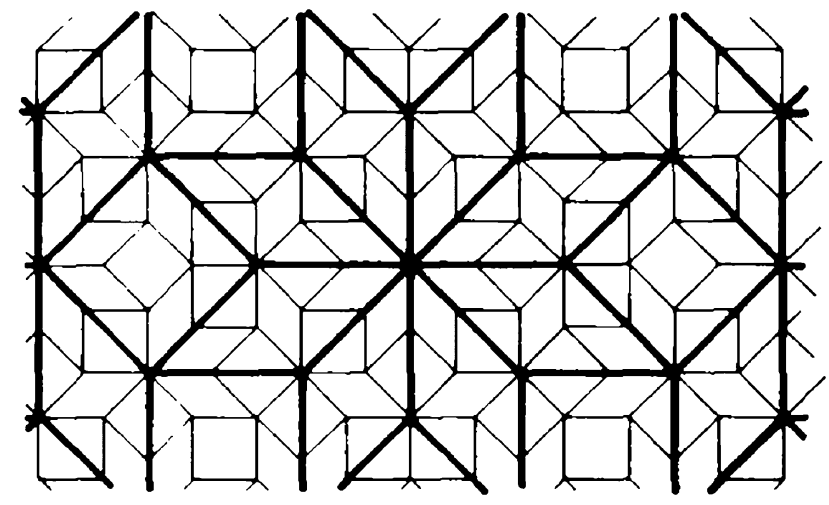

(a)

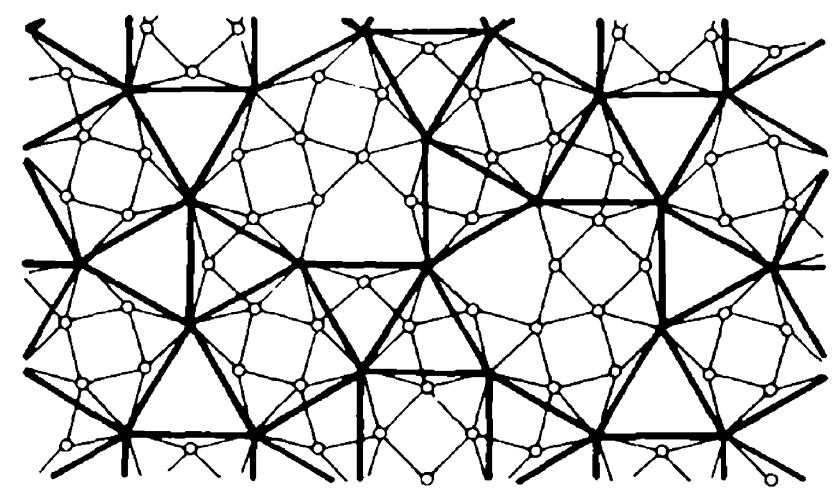

(b)

Figure 34

(a) Two-dimensional octagonal tiling and the same scaled with a factor $1+\sqrt{2}$. (b) Dodecagonal tiling and the same transformed with rotoscaling, with factor $\sqrt{2}+\sqrt{3}$ and rotation over $15^{\circ}$. matrix that commutes with all point group elements belongs to the so-called centralizer. As a consequence, this matrix leaves the two subspaces invariant, because the two irreducible components are non-equivalent. In each subspace the action is a multiplication with a certain factor. As an example, here we consider two matrices

$$
A=\left(\begin{array}{cccc}
0 & 0 & 1 & 1 \\
-1 & -1 & -1 & 0 \\
0 & -1 & -1 & -1 \\
1 & 1 & 0 & 0
\end{array}\right), B=\left(\begin{array}{cccc}
0 & -1 & -1 & -1 \\
1 & 1 & 0 & 0 \\
0 & 1 & 1 & 0 \\
0 & 0 & 1 & 1
\end{array}\right) .
$$

Matrix $A$ belongs to the centralizer, and has two invariant subspaces. In the first, the physical space, it acts as a multiplication with the factor $-1 / \tau$, and in the other, the internal space, with a factor of $\tau$. This means that the pattern of vertices of the tiling is invariant under a multiplication with $-(1+\tau)$. The inverse transformation does not leave the pattern invariant, because it decreases the distance in physical space and increases the distance in internal space. Since the atomic surfaces are finite, their intersection with the physical space will disappear, together with a vertex. The same action works in reciprocal space. However, the points of the Fourier module are projections of points of the reciprocal lattice. They do not disappear when the distance in internal space changes. Therefore, the set of points of the diffraction pattern is invariant under the scale transformation.

The other element, $B$, transforms every element of the point group into another element of the point group that is not necessarily the same. $B$ belongs to the so-called normalizer. The centralizer is a subgroup of the normalizer. Again because the two irreducible components are non-equivalent, the two spaces, physical and internal, are left invariant. Now the action is not simply multiplication, but multiplication combined with a rotation, i.e. a roto-scale transformation (Janner, 1991; Janssen, 1991, 1992). Fig. 34 shows two examples, one for a centralizer element on an octagonal tiling and one for a normalizer element on a dodecagonal one. The action on the points of the Fourier module are similar.

For icosahedral quasicrystals, the scaling factor is $\tau^{3}$ for Ptypes, and $\tau$ for F-types.

The points of a vector module with $n$ basis vectors, where $n$ is larger than the dimension of the physical space, form a dense set: there is no minimal distance. Nevertheless, the symmetry of the module can be used for the description of finite objects by selecting a finite subset of the vector module. An example is given in Fig. 35. The pentagon has corners at the vectors $\mathbf{e}_{i}(i=1, \ldots, 4)$ and $-\mathbf{e}_{1}-\mathbf{e}_{2}-\mathbf{e}_{3}-\mathbf{e}_{4}$. The star pentagon encloses another pentagon, which is $\tau^{2}$ smaller. The coordinates of the corners of the smaller pentagon are integers: they belong to the vector module spanned by the vectors $\mathbf{e}_{i}$. There is a scale transformation from the larger to the smaller pentagon. However, inverting the scale transformation would lead to points outside the original pentagon and eventually, if repeated, to a structure with an infinite number of points. 
Similar scale transformations occur for other geometric structures and some molecules. For example, snow crystals and viruses (Fig. 36) show this type of structural scaling (Janner, 2013). It should be noted that these transformations are not symmetry operations in the usual sense, because they do not leave the physical laws invariant, and they are only valid for a point-like structure: extended atoms in the molecule would be mapped on larger and larger atoms, which is non-physical.

\section{Physical properties of aperiodic crystals}

\subsection{Characterization of states in an aperiodic crystal}

The space-group symmetry of a crystal is very helpful for the study of electron and vibration states. These states satisfy Bloch's theorem. For electron states and phonons, this means

$$
\Phi(\mathbf{r})=\exp (\mathbf{i k} \cdot \mathbf{r}) \mathbf{U}_{\mathbf{k}}(\mathbf{r}), \quad \mathbf{u}_{\mathbf{n j}}=\exp (\mathbf{i k} \cdot \mathbf{n}) \mathbf{e}(\mathbf{k}, \mathbf{j}),
$$

respectively, where $\mathbf{U}_{\mathbf{k}}(\mathbf{r})$ is lattice periodic, and therefore is determined by its value in the unit cell, and where $\mathbf{j}$ is the number of vibrating atoms in the unit cell, the atoms are labelled with the lattice vector $\mathbf{n}$ and $\mathbf{j}$. The Bloch Theorem is also valid for aperiodic crystals, and the electron and vibration states are determined by the values in the $n$-dimensional unit cell in superspace. However, the simplification of the problem for aperiodic crystals is not as important as for lattice periodic crystals. This is partially due to the number of degrees of freedom (for phonons) and to the complexity of the states (for electrons and phonons), which make it necessary to use very high harmonics in a Fourier series. This makes the study of physical problems for aperiodic crystals more complicated than that for three-dimensional lattice periodic crystals. Moreover, there is a number of special features that make the physical properties of aperiodic crystals more interesting. We shall deal here with a number of special properties which follow from the aperiodicity.

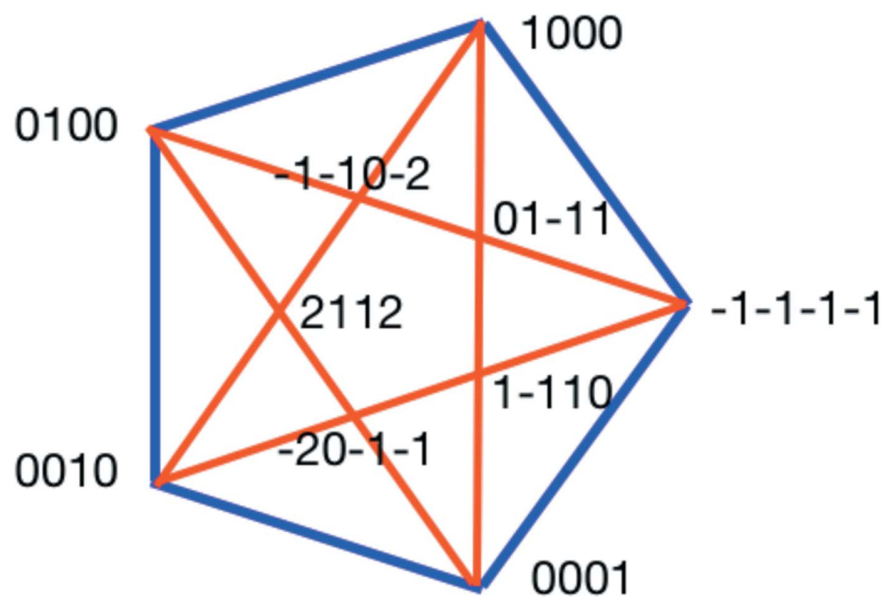

Figure 35

The points of the pentagram may be labelled with integer indices with respect to the basis of the Fourier module for the Penrose tiling.

\subsection{Tensorial properties}

Macroscopic properties of crystals may be described using tensors. These properties transform under elements of the point group, and knowledge of the symmetry elements puts, in general, restrictions on the components of the physical property and may even allow or forbid such a property. This is not different for aperiodic crystals, where the point group acts on vectors in superspace. The vectors transform with a representation of the point group, called the vector representation, which is, in general, reducible into irreducible components. For aperiodic crystals, the vector representation has at least irreducible components in physical and internal space.

We take as an example the metric tensor. Its components, $g_{i j}$, are the scalar products of pairs of basis vectors: $g_{i j}=\mathbf{a}_{i} \cdot \mathbf{a}_{j}$. It is a second-rank symmetric tensor which transforms with the symmetrized square of the vector representation. It should be invariant under the point group, in this case, the point group of the superspace group. As an example, consider the icosahedral point group $532\left(5^{2} 32\right)$. It has five conjugation classes, with representatives the elements 1 (the identity), $5\left(5^{2}\right), 5^{2}\left(5^{4}\right), 3(3)$ and 2(2). This representation is reducible into two components, the physical and the internal components. The symmetrized square of this representation has characters $21,1,1,0,5$ containing twice the identity representation. This means that the metric tensor for the point group has two free parameters.

Another example is the electric gradient tensor $\partial \mathbf{E}_{j}$. For the incommensurate phase of $\mathrm{Rb}_{2} \mathrm{ZnBr}_{4}$, this was analyzed in van Beest et al. (1982). One may distinguish two contributions, in physical space $\left(t_{i j}^{\mathrm{E}}\right.$ with $\left.i=1,, 3\right)$ and in internal space $\left(t_{i j}^{\mathrm{I}}\right.$ with $i=4)$. The superspace group is $\operatorname{Pcmn}(00 \gamma) s s 0$. The point

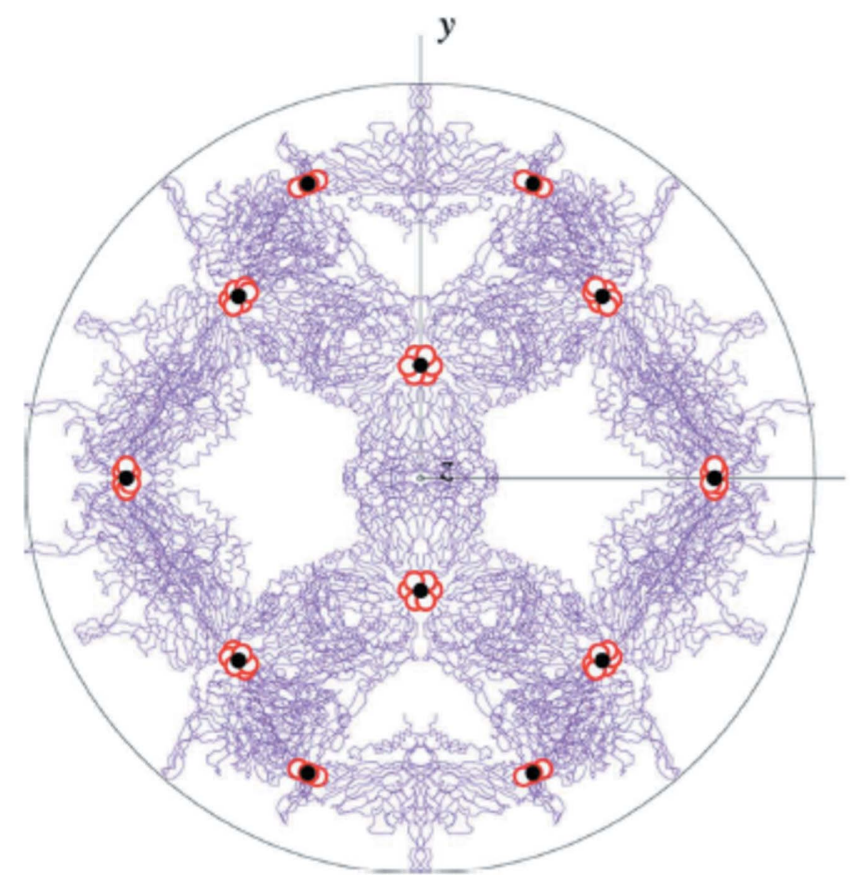

Figure 36

Pariacoto virus, dodecahedral orbit $\mathrm{A} 12_{2}$ (black points), generator [100011]. Residue Asn173 of chain C (Janner, 2013). 
group $\mathrm{mmm}$ has eight elements and eight irreducible representations. The electric field has values in the physical space, the gradient may be in physical and internal space. The tensor $t_{i j}^{\mathrm{E}}$ then has three free parameters and $t_{i j}^{\mathrm{I}}$ has one free parameter. The tensor elements may be obtained by a projection technique or by solving the equation $g t=t$ for generators $g$ of the superspace group.

The elasticity tensor $\epsilon^{\mathrm{el}}$ connects the strain with the stress. The strain for each atom gives a displacement vector in superspace. One may distinguish components $\mathbf{v}(\mathbf{r})$ in physical space and $\mathbf{w}(\mathbf{r})$ in internal space. Then the strain components are

$$
\begin{aligned}
e_{i j} & =\left(\partial_{i} \mathbf{v}_{j}+\partial_{j} \mathbf{v}_{i}\right) / 2(i, j=1,2,3) ; \\
f_{i k} & =\partial_{i} \mathbf{w}_{k}(i=1,2,3 ; k=4, \ldots, n) .
\end{aligned}
$$

The elastic energy has three components: one from the coupling between physical strain components, one from that for internal components, and one for the coupling between internal and physical components.

$$
F=\frac{1}{2} \sum_{i j k \ell} c_{i j l \ell}^{\mathrm{E}} e_{i j} e_{k \ell}+\frac{1}{2} \sum_{i j k \ell} c_{i j l \ell}^{\mathrm{I}} f_{i j} f_{k \ell}+\sum_{i j k \ell} c_{i j l \ell}^{\mathrm{EI}} e_{i j} f_{k \ell}
$$

For the icosahedral group, the representation theory gives five independent components: two for $e^{\mathrm{E}}$, two for $e^{\mathrm{I}}$ and one for $e^{\mathrm{EI}}$. Together there are five elastic constants for icosahedral quasicrystals. The superspace approach also provides the means for a more detailed analysis. A more detailed discussion can be found in Vol. D of International Tables for Crystallography (Janssen, 2003a,b; Ephraïm et al., 2003).

\subsection{Phonons}

For collective small vibrations around the equilibrium positions, called phonons, these are labelled by the wavevector $\mathbf{k}$ and a branch label $v$. For lattice periodic structures Bloch's Theorem reduces the problem from an infinite one to a problem with three times the number of atoms in the unit cell degrees of freedom. For aperiodic crystals this also holds, but the number of degrees of freedom is still infinite, because the atoms correspond to points on the atomic surfaces. To study the vibrations, we have to know the equilibrium positions. This asks for an explanation of the formation of an aperiodic ground state. $A b$ initio calculations are quite difficult. Therefore, simple models have been studied.

The origin of a modulated phase may be explained with such a model. One model is the Discrete Frustrated $\Phi^{4}$ (DIFFFOUR) model. In one dimension it is a chain with energy given in terms of the deviations $\left(u_{n}\right)$ of the atoms from an equidistant array.

$$
E=\sum_{n}\left(\frac{\alpha}{2} u_{n}^{2}+u_{n}^{4}+\frac{\beta}{2}\left(u_{n}-u_{n-1}\right)^{2}+\frac{\delta}{2}\left(u_{n}-u_{n-2}\right)^{2}\right) .
$$

The phonon branches in the chain depend on the parameters $\alpha, \beta, \delta$, which can be considered to be temperature dependent. In particular, $\alpha$ can effectively depend on temperature, because of the fourth-order term. For higher temperature, the deviations become larger, and the effective value of $\alpha$ will change.

Variation of the parameters makes the phonons of the equidistant array $\left(u_{n}=0\right)$ become unstable. The soft mode leads to a modulation wave with wavevector $q=\operatorname{Arc} \operatorname{Cos}(\beta / 4 \delta)$. In general, this is an incommensurate displacement wave for a certain range of values of the parameters. One of the parameters for the calculation of the phase diagram may be eliminated. For these two new parameters (called $A / D$ and $B / D$ ), the phase diagram is given in Fig. 37. $\mathrm{P}$ represents the para-phase $\left(u_{n}=0\right.$ for all $\left.n\right)$; in the regions below the phase transition line incommensurate phases (hatched regions) and commensurate regions can be found, with rational values of $q$ ranging from 0 (ferro-structure) to $1 / 2$ (anti-ferro-structure). Lowering $A / D$ shows that all incommensurate phases become commensurate via a so-called lockin transition. The parameters $\alpha, \beta$ and $\delta$ (and $A / D$ and $B / D$ ) may be fit to measured interaction parameters for a material. This determines the ranges of the parameters, and shows whether a material has such an incommensurate phase and whether there is a lock-in transition or not.

Using a model in two or three dimensions, and more interactions may give a model with more complex transitions, e.g. from a phase with one modulation wavevector to a phase with two modulation wavevectors.

A related version of the DIFFFOUR model has a potential energy

$$
V=\sum_{n} \sum_{j=1}^{3}\left(\frac{\alpha_{j}}{2}\left(u_{n}-u_{n-j}\right)^{2}+\frac{1}{4}\left(u_{n}-u_{n-1}\right)^{4}\right),
$$

with up to a third-neighbor interaction. The three spring constants are $\alpha_{1}, \alpha_{2}, \alpha_{3} . V$ only depends on differences. This version of the DIFFFOUR model shows that at the phase transition towards the modulated phase the modes at $\pm q$ go

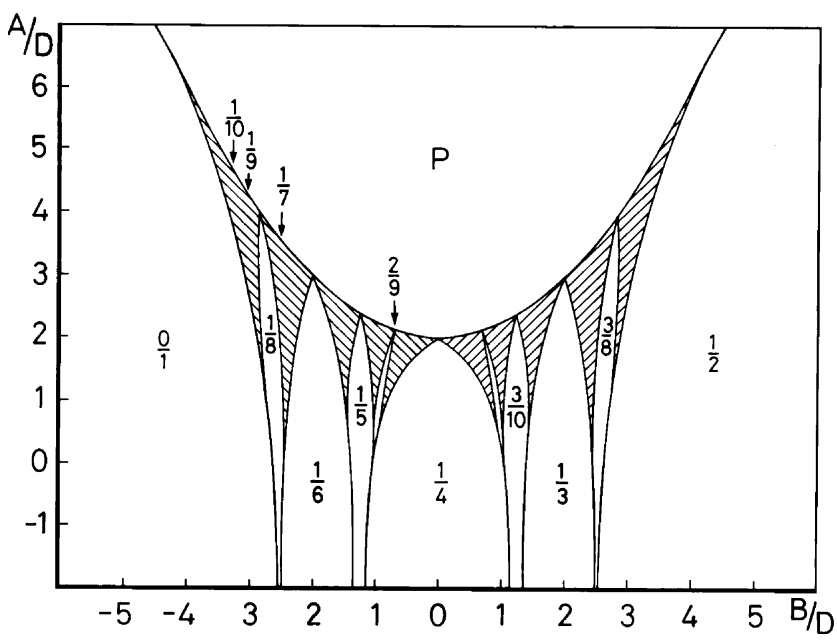

Figure 37

Phase diagram of the DIFFFOUR model as a function of the parameters $A / D$ and $B / D$, which depend in a simple way on the values of the spring constants. $\mathrm{P}$ is the para-phase $\left(u_{n}=0\right)$. Rational values of the wavevector, with respect to the basis in reciprocal space, give superstructures. The hatched regions are incommensurate phases. 
simultaneously to zero and give rise to two modes in the modulated phase. One is the $q=0$ acoustic mode, the rigid displacement of the chain, which has frequency $(\omega)$ equal to zero. From the critical wavevector acoustic-mode-like dispersion curves start. The other mode is a phason, which will be discussed in the next section.

The character of vibrations in incommensurate phases may be seen from even simpler models. One is the Modulated Chain Model (MCM). It assumes that the coupling between atoms depends on their position with respect to the modulation wave. The potential energy in this model is

$$
V=\sum_{n} \frac{\alpha_{n}}{2}\left(x_{n}-x_{n-1}-a\right)^{2} .
$$

Here $x_{n}$ is the position of the $n$th atom and $a$ the lattice constant. The coupling parameter $\alpha_{n}$ is a periodic function. An example is $\alpha_{n}=\alpha_{0}(1-\Delta \cos (q \cdot n a))$. The (unphysical) limit of $\Delta=1$ shows a spectrum with a very hierarchical ordered fractal ( $c f$. Fig. 38 for $\Delta=0.3$ ). Moreover, the eigenvectors also have a structure that differs strongly from that seen for periodic chains. The situation is comparable to that for electrons. We shall come back to the properties of spectra and eigenvectors in $\$ 5.5$.

A simple model for incommensurate composites is the model of Frank and Van der Merwe (FvdM; Frank \& van der Merwe, 1949), an incommensurate version of the FrenkelKontorova model, which actually goes back to 1929 (Dehlinger, 1929). It consists of a linear chain with lattice constant $a$ and nearest-neighbor interaction, in a periodic background potential with periodicity $b$. Its potential energy is

$$
V=\sum_{n}\left(\frac{\alpha}{2}\left(x_{n}-x_{n-1}-a\right)^{2}+\lambda \cos \left(2 \pi \frac{x_{n}}{b}\right)\right) .
$$

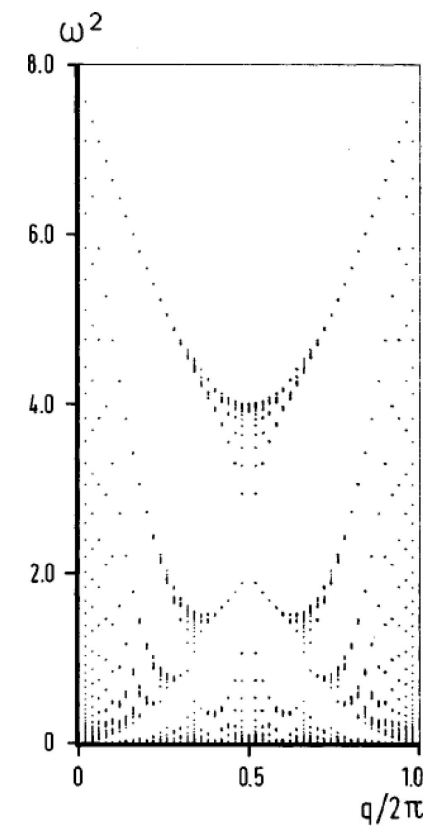

Figure 38

Spectra for the Modulated Chain Model (MCM) as a function of the modulation wavevector $\alpha_{n}=(1-0.3 \cos (q \cdot n a))$.
The effect of the interaction between chain and background potential is a modulation. The positions of the atoms are $x_{n}=n a+f\left(2 \pi x_{n} / b\right)$ with a modulation function $f$ with periodicity 1 . For small values of $\lambda$, the function is sinusoidal, for large values it is discontinuous, and in between there is a phase transition from continuous to discontinuous. The diffraction peaks appear at the positions $2 \pi(h / a+k / b)$, which has rank two.

A slightly different model is the Double Chain Model. It consists of two chains with a nearest-neighbor interaction in each chain and an interaction between atoms of different chains. The incommensurability is a consequence of the fact that the lattice constants for the two chains, $a$ and $b$, are incommensurate: $a / b$ is irrational. Because of the inter-chain interaction, each chain is modulated with the periodicity of the other chain. The main gain with respect to the FvdM model is in the dynamics. The FvdM model does not have translation symmetry, responsible for a zero frequency vibration mode, while this mode exists for the Double Chain Model. In the latter there would be two dispersion curves, with two acoustic branches if the inter-chain interaction vanishes. By the interaction the two curves become mixed. The two acoustic branches from the uncoupled system, which are long wavelength modes, give two branches of a new type. One describes long wavelength waves in both chains in the same direction, and the other long wavelength modes where one chain moves in the opposite direction to the other. The latter modes are called sliding modes or phasons.

The simple models mentioned above have been studied to obtain a view of the phonon excitations. The frequencies, the eigenvalues of the dynamical matrix, cannot be solved

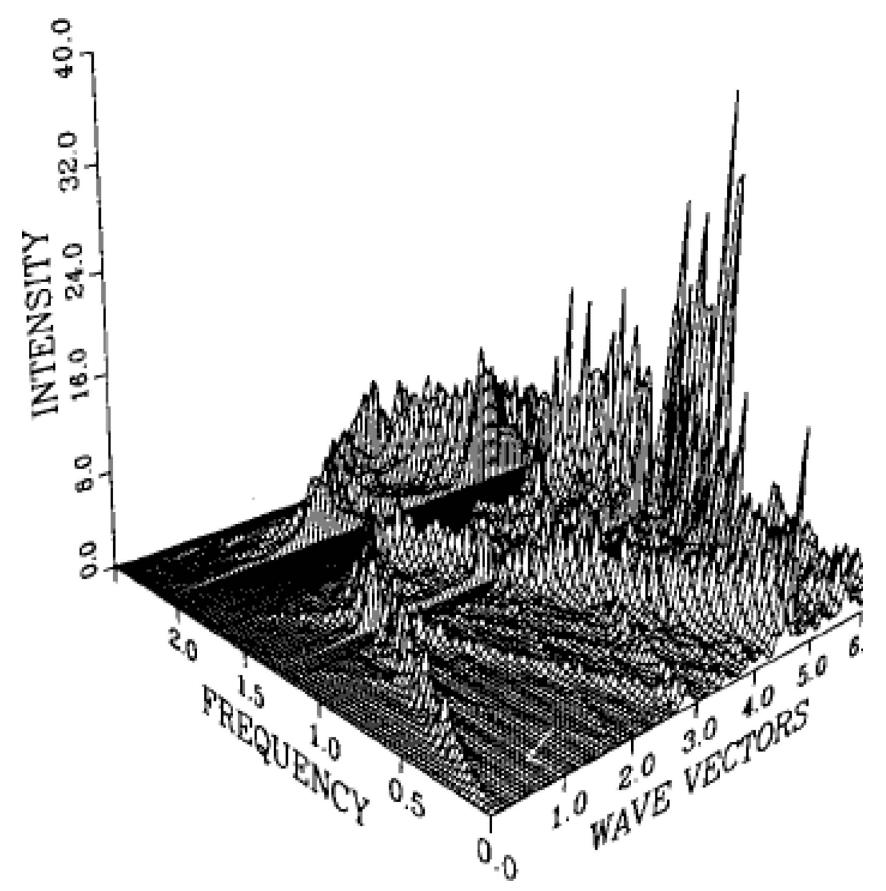

Figure 39

Scattering intensity from phonons in the Fibonacci chain, as a function of the wavevector and the frequency (Quilichini \& Janssen, 1997). 


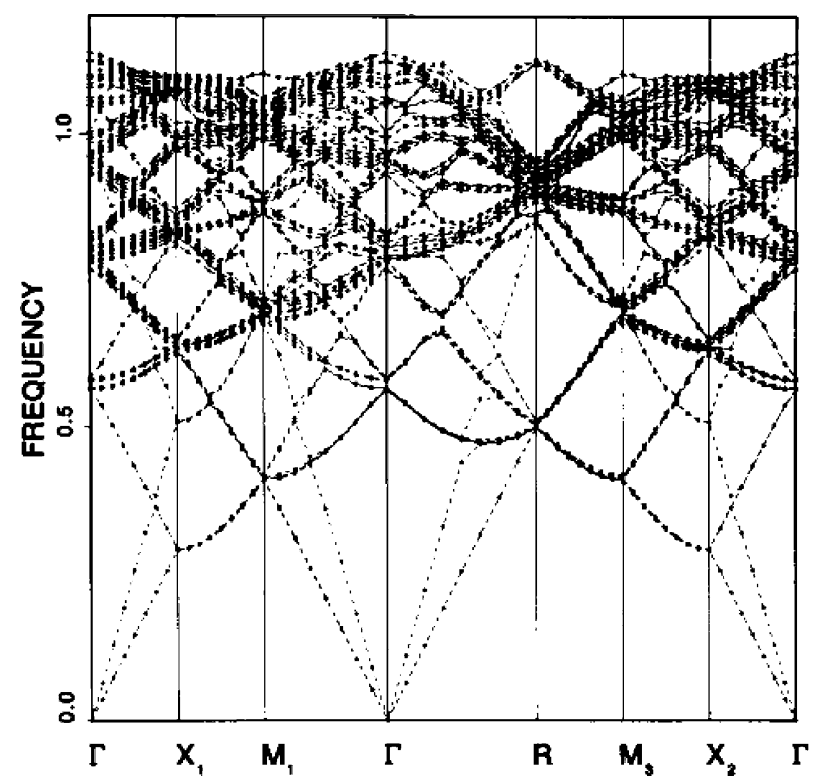

Figure 40

Dispersion curves for the $3 / 2(\sim 1+\tau)$ approximant to the threedimensional icosahedral tiling (Los et al., 1993a,b). The letters give positions in the Brillouin zone.

numerically because the matrix is infinite. The dynamical properties for approximants can be solved by choosing periodic boundaries in the DIFFFOUR model or rational values of $a / b$ in the case of the FvdM model. One finds that acoustic modes still exist for the DIFFFOUR model, and that in one dimension the spectrum has many gaps.

To obtain a first insight for quasicrystals tiling models may be considered, approximating these with lattice periodic tilings. This has been done for the Fibonacci chain, Penrose tiling, Ammann-Beenker tiling and the three-dimensional icosahedral tiling. For three-dimensional lattice periodic crystals, the phonon dispersion curves have the periodicity of the reciprocal lattice. For aperiodic crystals, there are phonon branches growing from each wavevector in reciprocal space, albeit with very different intensity in the scattering of neutrons. Globally, stronger branches are associated with stronger peaks in the structure factor (Quilichini \& Janssen, 1997). This is illustrated in Fig. 39. The branches meet on the perpendicular plane through the midpoint between two strong peaks, as on the border of a Brillouin zone. A volume in reciprocal space bounded by such planes is called a pseudoBrillouin zone.

For the two-dimensional tiling models, in the Brillouin zone of approximant 2 there are acoustic branches, and many optical branches. The latter are fairly flat. With higher approximants (better approximants) a very similar picture is seen which scales with the preceding curves. The Brillouin zone shrinks to zero for the aperiodic tiling. The same result has been obtained for three-dimensional models (Los et al., $1993 a, b)$. The dispersion curves show acoustic branches and many rather flat optic branches (see Fig. 40).

For real quasicrystals, the numerical problem is bigger. If approximants are used, the number of atoms increases rapidly. The simple interactions used for the tiling models can no longer be used. Good approximants to the interaction potentials have been constructed by Mihalkovič and collaborators (Mihalkovič et al., 2008). These are oscillating potentials of the form

$$
V(r)=\frac{C_{1}}{r^{\eta_{1}}}+\frac{C_{2}}{r^{\eta_{2}}} \cos (k \cdot r+\phi) .
$$

Here the parameters depend on the atom types between which the interaction is taken into account.

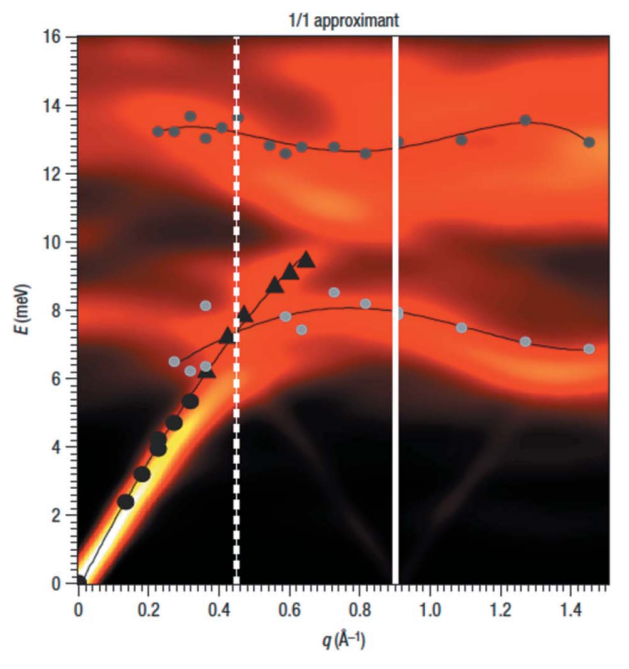

(a)

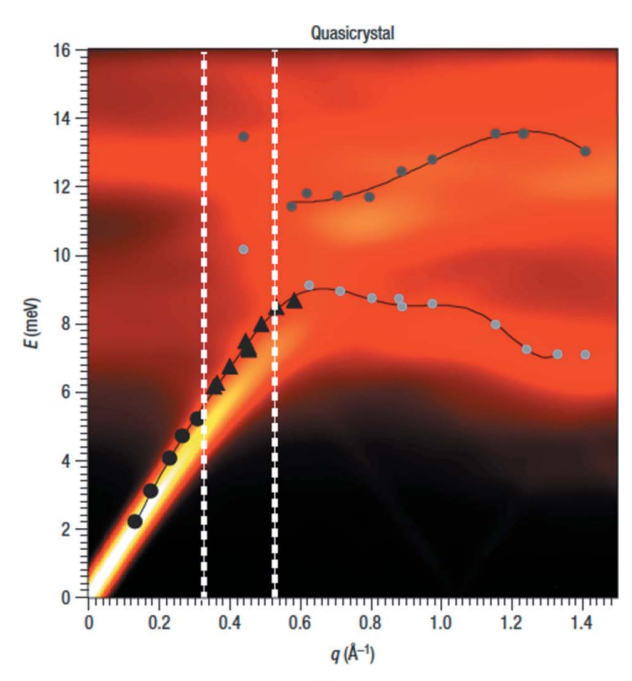

(b)
Figure 41

Dynamical structure factor $S(q, \omega)$ for i-ZnMgSc. Experiment and calculations compared. (a) 1/1 approximant; $(b)$ quasicrystal. The results of the calculation (2/1 approximant for the quasicrystal) are shown in the background distribution. The experimental acoustic curves are given by full dark circles and triangles. Optical branches are represented by the other symbols. The vertical lines indicate the Brillouin Zone and pseudo-Brillouin Zone boundaries (after de Boissieu et al., 2007). Reproduced with permission from Macmillan Publishers Ltd. Copyright 2007. Using such potentials, the dispersion for an approximant to a binary quasicrystal has been calculated. Of course, this cannot be compared with the quasicrystal itself, but one has compared a first and second approximant. The results are in quite good agreement with experiment (de Boissieu et al., 2007), see Fig. 41.

An earlier review on the lattice dynamics of quasicrystals is given by Quilichini \& Janssen (1997), and a more recent one by Janssen \& de Boissieu (2014).

\subsection{Phasons}

An incommensurate modulated phase often results from the softening of a phonon. The phonons are characterized by a wavevector $\mathbf{k}$ 
and a band index, $v$. If the frequency $\omega_{\mathbf{k}_{0} v}$ goes to zero, by symmetry the mode $v$ at $-\mathbf{k}_{0}$ also goes to zero. Below the phase transition, where a modulation wave is created, the modes at $\mathbf{k}_{0}+\Delta \mathbf{k}$ and $-\mathbf{k}_{0}+\Delta \mathbf{k}$ are coupled. Then two branches originate from the points $\pm \mathbf{k}_{0}$. One branch describes a shift of the modulation wave with respect to the crystal (varying along the crystal with $\Delta \mathbf{k}$ ), and the other one describes a change of the amplitude of the modulation wave, also varying with $\Delta \mathbf{k}$. The first one is called the phason branch, the other the amplitude branch. The phason branch has a simple interpretation in terms of superspace. Since the phase corresponds to the internal degree of freedom, a phason is a displacement along the internal space (see Fig. 42). The term phason was coined by Overhauser (1971). He considered charge-density waves (CDW). When the CDW has an incommensurate relation to the underlying crystal, it may move without friction. This is directly related to the phason in an incommensurate modulated phase, because the interaction between CDW and lattice will create an incommensurate lattice distortion. Just as phonons, phasons may become soft. A probable example is a phase transition in TTF-TCNQ (see $\S 3.5)$.

Long-wavelength phasons can also be described in the context of hydrodynamic theory. They are the consequence of the loss of symmetry, comparable to a Goldstone mode. In this theory there are as many new modes as the dimensions of internal space (Bak, 1985b; Lubensky et al., 1985).

For modulated phases, the phason and amplitude modes originate from two modes above the phase transitions. The number of modes, consequently, does not change. Phasons occur in an analogous way in other types of aperiodic crystals. For quasicrystals, these modes have a slightly different character. Because the atomic surfaces are discontinuous, the motion of the atoms is not smooth. When the border of an atomic surface is passed when the physical space undulates, the atoms jump from one position to another. These are called phason jumps.

The phason in aperiodic composites is also called a sliding mode. For $q=0$, one subsystem moves with respect to the

Figure 42

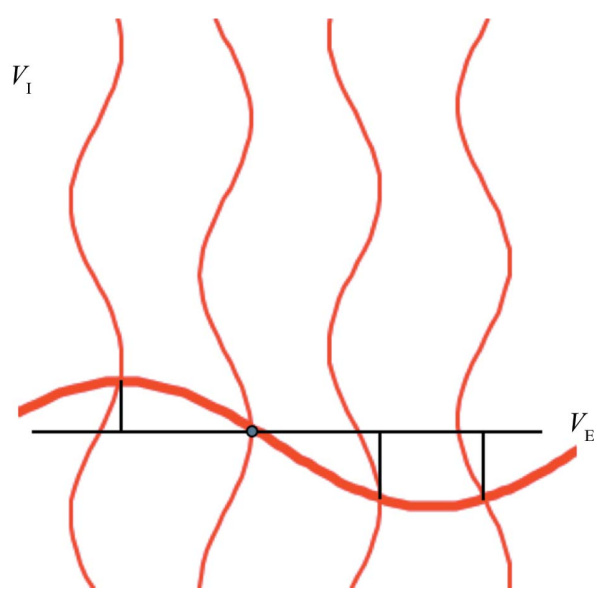

Phason in an incommensurate modulated phase, represented in superspace. $V_{\mathrm{E}}$ is the physical (external) space, $V_{\mathrm{I}}$ is the internal (perpendicular) space. other(s). For long wavelength phasons, the direction of this sliding changes periodically, with a long period. Phasons in composites have not yet been studied in detail.

Phason jumps have been studied in quasicrystals. The phason jump may occur as an individual motion or as a collective phason excitation. Individually, a jump may occur between positions that are energetically almost the same. A simple example is given in Fig. 43 for a Penrose tiling. They have been observed in real quasicrystals (Coddens et al., 1991). However, in complex alloys, that are not quasicrystals, one has seen similar jumps at defects (Dolinsšek et al., 2002). Correlated phason jumps have also been observed (Coddens et al., 1997). The collective phase jumps are the phasons. For quasicrystals these can be modeled with a hydrodynamic theory, but this is not an atomistic description. It predicts an over-damped phason mode with $\omega \sim i q^{2}$. This has been verified with coherent X-ray photo-correlation spectroscopy (de Boissieu, 2012).

The phason is usually over-damped not only in quasicrystals, but also in incommensurate modulated phases. This is due to the non-linear character of the interactions, which are essential for the incommensurate state. This is even more so for aperiodic crystals with discontinuous atomic surfaces. Then finite jumps lead the system out of the harmonic domain.

A recent overview of phonons and phasons in quasicrystals is given in de Boissieu (2012).

\subsection{Electrons}

For electron states, the problem remains infinite, even in three dimensions, because the wavefunctions inside the unit cell have to be determined. One way of doing this is to approximate the lattice periodic wavefunctions $\mathbf{U}_{\mathbf{k}}(\mathbf{r})$ by a truncated Fourier series. This is also possible in superspace, but it turns out that wavefunctions often have very detailed structure and consequently high Fourier components are needed. A second procedure is to use an approximant, or better a series of approximants (\$4.9).

Also here a first insight can be obtained from simple models. One is the tight-binding model for a linear chain. It considers a chain of atoms, with one state, $\mid n>$, per atom. The energy is given by

$$
H=\sum_{n}\left(a_{n}|n><n|+b_{n}|n><n-1|\right) .
$$

The off-diagonal version takes $a_{n}=0$ and $b_{n}=A$ or $=B$, depending on whether the interval between $n$ and $n-1$ is long or short.

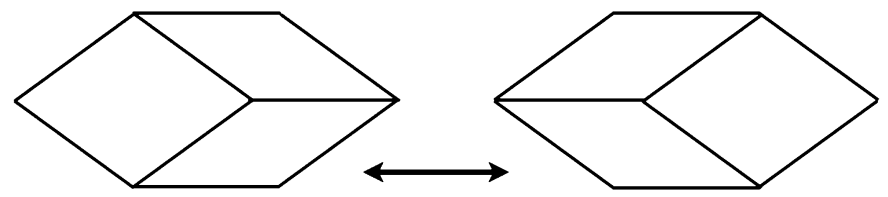

Figure 43

Jump between two vertex configurations in a Penrose tiling. 
A second model, which stems from a square lattice in an external magnetic field, leads to the, so-called, almost Mathieu equation. The discrete Schrödinger equation becomes

$$
\Psi(n+1)+\Psi(n-1)+2 \lambda \cos (2 \pi n \alpha-\phi) \Psi(n)=E \Psi(n),
$$

where $\alpha$ is an irrational number.

These models show that, at least for linear chains, the spectrum is as complex as that for the MCM model for phonons. In principle, the electron states belong to one of three types. They may be extended (i.e. they do not tend to zero for $n$ approaching infinity), localized (exponential decay for $n \rightarrow \infty$ ) or critical (tending to zero with a power law). As previously mentioned, in three-dimensional lattice periodic systems the states are extended and localized states may occur at impurities or dislocations. By choosing a proper substitution rule for a substitutional chain, all types of states may be found. However, it is not clear whether this also holds for two- and three-dimensional aperiodic crystals.

For real aperiodic crystals, ab initio calculations have been used. As for phonons, the calculations are restricted to approximants and these are necessarily limited. For details, refer to Krajči \& Hafner (1997a,b), Zijlstra et al. (2005), Ishii \& Fujiwara (2008) and Trambly de Laissardière \& Mayou (2014).

Calculations on simple models and the absence of a threedimensional Brillouin zone are reason to believe that aperiodic crystals could have interesting new properties, which eventually could lead to new applications. An example is the pseudo-gap in the electron density of states near the Fermi surface. This is responsible for remarkable electric and mechanical properties, such as the conduction and friction at the surface.

\subsection{Stability}

Incommensurate phases are often intermediate between a three-dimensional lattice periodic structure and a superstructure. This illustrates the question about the stability of these phases. As can be seen from Fig. 37, for very low values of $A / D$ the structures are lattice periodic. With increasing values, which is associated with increasing temperature, an incommensurate phase may occur and after that there is a transition to the parent structure, which is lattice periodic. It depends on the value of $A / D$ for temperature $T=0$ and whether at that temperature an incommensurate state is stable. Higher values of $A / D$ mean higher temperature, and an increase of the entropy term in the free energy. Therefore, the stability of an aperiodic crystal phase is the result of a balance between energy and entropy.

The same holds for quasicrystals. Some authors have claimed that a quasicrystal can only exist at higher temperature, and that the entropy term in the free energy determines the stability of the quasicrystalline phase. Some models seem to support that idea. However, there is no reason to believe that aperiodic crystals, and quasicrystals in particular, are always stabilized by entropy.
Incommensurate phases sometimes appear by the interaction between electrons and nuclear structure. This is the socalled Peierls mechanism. If the Fermi energy is in the middle of an energy band, a distortion producing a doubling of the unit cell may lower the occupied band and lift the unoccupied band. Therefore, the stability is determined by the position of the Fermi level. A similar reasoning may lead to a distortion with another wavevector, eventually an incommensurate one.

A similar mechanism may be at work for quasicrystals. It has often been found that the density of states at the Fermi level is very low, a pseudo-gap at the Fermi level. The position of the Fermi level is influenced by distortion of the lattice, for example an icosahedral structure in the fluid, and by the number of electrons in the band, the ratio between the number of electrons and atoms. This ela ratio seems to be very important for the stability of quasicrystals (Mizutani et al., 2012; Tsai, 2013). For unstable quasicrystals, the composition range is relatively broad, but for stable icosahedral crystals, it is rather narrow. For three families, the precise values of $e / a$ are shown in Fig. 19.

\subsection{Magnetism}

Simple models can explain the existence of incommensurate spin waves. In Enz (1960) and Elliott (1965) a generalized Ising model was introduced with nearest- and next-nearestneighbor interactions. Competition between the two forces may result in an incommensurate spin wave. In Janssen (1972), exchange and dipolar interactions were used in a model for Eu-chalcogenides, which also includes spin-lattice interactions. The presented results were commensurate, but with a relatively large denominator (Fig. 44). In fact, this was an example of a modulated structure with a common wavevector for lattice and magnetic structure.

An often studied model was the axial next-nearest neighbor Ising (ANNNI) model. Its Hamiltonian is

$$
H=\sum_{\mathbf{n}}\left(J_{0} S_{\mathbf{n}} S_{\mathbf{n}-\mathbf{a}}+J_{0} S_{\mathbf{n}} S_{\mathbf{n}-\mathbf{b}}+J_{1} S_{\mathbf{n}} S_{\mathbf{n}-\mathbf{c}}+J_{2} S_{\mathbf{n}} S_{\mathbf{n}-2 \mathbf{c}}\right) .
$$

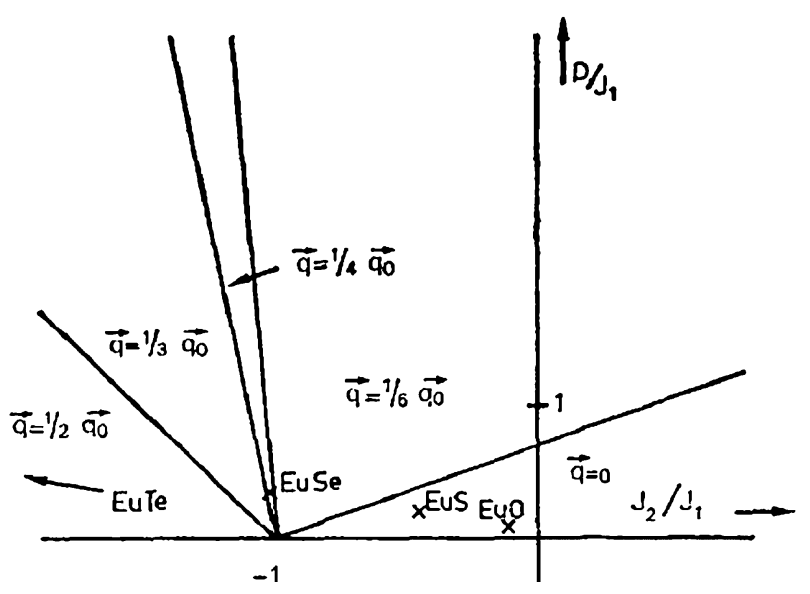

Figure 44

Regions in parameter space (pressure and ratio of first and second neighbour interaction) where (rational) values of the magnetic wavevector are stable (Janssen, 1972). 
Its phase diagram and properties were extensively studied (Selke, 1988), see Fig. 45, showing many phases between ferromagnetic and anti-ferromagnetic phases. There are commensurate phases in the figure indicated by a series of up and down spins $(\langle 34\rangle$ means +++---- with period 7 , for example). In between, there are incommensurate phases such as those for the DIFFFOUR model (Fig. 37).

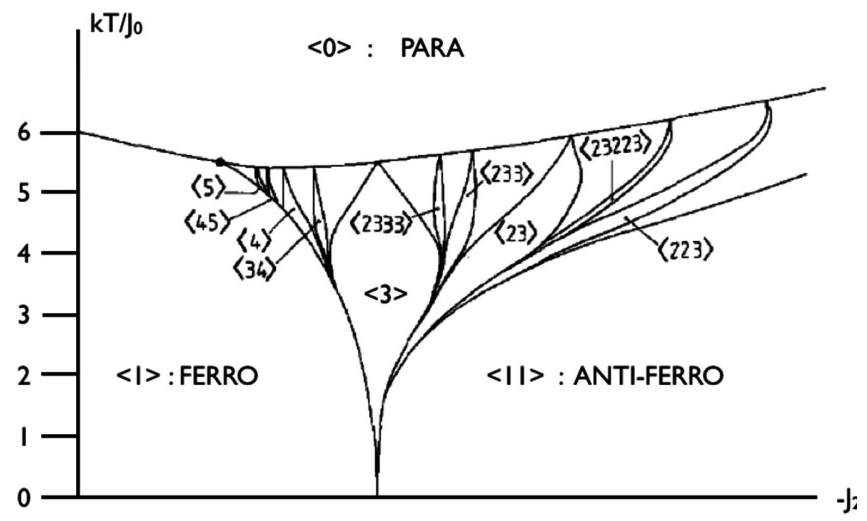

Figure 45

Phase diagram of the three-dimensional ANNNI model (after Selke \& Duxbury, 1984). Compare this with Fig. 37. Indicated are a number of commensurate phases. They are characterized by the number of spins of the same sign: $\langle 2333\rangle$ is a period 11 wave with structure ,,,,,,,,,,++---+++--- etc. Close to the transition line from the para-phase (no magnetization) there are incommensurate phases.

\section{Surface Structure of Al-Cu-Fe}
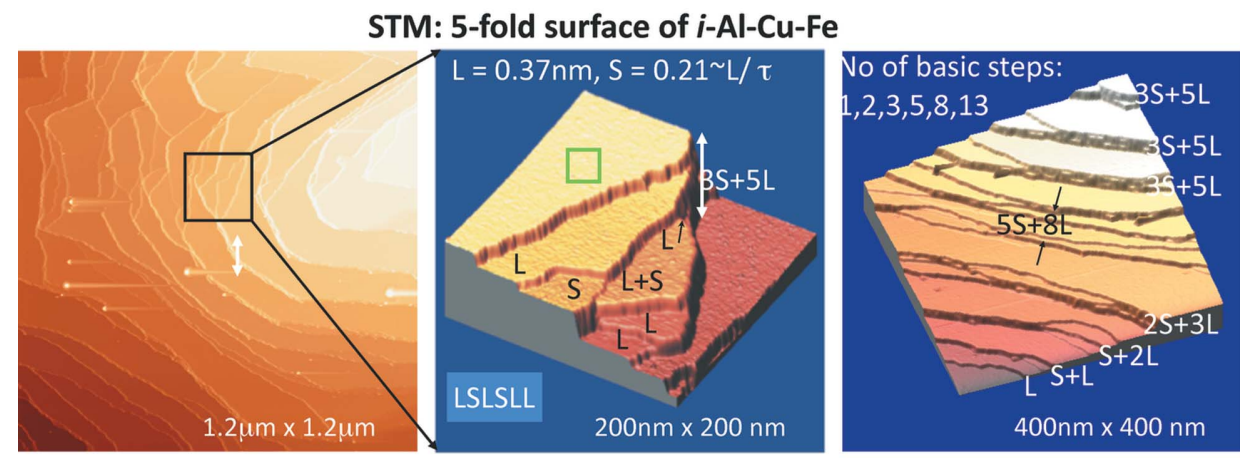

$\mathrm{Ar}^{+}$sputtering + annealed at $1023 \mathrm{~K}$

Sharma et al., Phys. Rev. Lett., 2004

$>\mathrm{L}$ and $\mathrm{S}$ steps appear in a Fibonacci sequence

$>$ Step heights: $\mathrm{H}=\mathrm{mS}+\mathrm{nL}$ with $(\mathrm{m}, \mathrm{n})$ integers

$>$ Steps bunch in Fibonacci numbers

$>$ Atomic structure can be checked down to atomic level

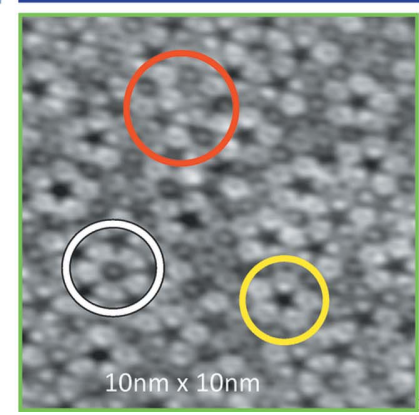

Figure 46

Steps on a fivefold surface of i-AlCuFe show a Fibonacci sequence. Courtesy of Dr H. R. Sharma (after Sharma et al., 2004).

\subsection{Surfaces}

Aperiodic crystals show, like lattice periodic crystals, flat surfaces. For quasicrystals, the study of these surfaces gives insight into the bulk structure. TEM pictures of icosahedral quasicrystals along a fivefold axis show locally fivefold symmetric structures, which can often be matched with a Penrose tiling.

A particular aspect is the occurrence of steps. A slightly tilted surface shows steps, for which one may observe two types, with two different step heights, with a mutual ratio equal to $\tau$. Moreover, along a line on the surface these steps are ordered according to a Fibonacci sequence: LSLLSLSL... (see Fig. 46).

A peculiar physical property of surfaces of an icosahedral quasicrystal is the low friction coefficient, which has been related to the existence of a pseudo-gap in the electronic structure. For decagonal phases, this same property has been found. In this case the friction along a surface through the periodic axis was very anisotropic with higher friction along the periodic axis.

Like all crystals, quasicrystals have localized electron states near the surface. For a model of a quasicrystal, a two-dimensional Penrose tiling, it has been shown that the energy level depends on the internal space variable (Zijlstra et al., 1999). In real systems, this has been verified: one has found topological surface states (Verbin et al., 2013).

A further special property of a quasicrystalline surface is that there are specific positions which are very well suited for catalysis.

In short, at the surface the aperiodicity leads to interesting phenomena (McGrath et al., 2010).

\section{Summary and outlook}

\subsection{Further reading}

In recent years a number of books and review articles have appeared. Clearly, there was some consensus that the field of aperiodic crystals had reached a level where it was possible to give a general view. After our own review papers (Janssen \& Janner, 1987; Janssen, 1988), Yamamoto gave an extensive crystallographic review (Yamamoto, 1996), dealing with modulated and composite aperiodic crystals and quasicrystals. There are several books dealing exclusively with quasicrystals: Janot (1997), Dubois (2005), Fujiwara \& Ishii (2008) and Steurer \& Deloudi (2009). The crystallography of modulated phases and composites was treated in van Smaalen (2007). 
A book dealing with the whole field of aperiodic crystals is Janssen et al. (2007).

\subsection{Further topics}

We have given an overview of the development of the crystallography of aperiodic crystals. The intriguing structure of aperiodic crystals has, in the last 50 years, interested many scientists. The structure of aperiodic crystals discussed here was based on the use of the superspace. This has turned out to be very useful. The structure also has important consequences for their physical properties. Therefore, we have given a short overview of those aspects as well.

Among the aspects we did not discuss in detail are the morphology, and the role of scaling transformations. As mentioned in $\$ 2$ the morphology of a conventional crystal is determined by the lattice planes, and consequently by the reciprocal lattice vectors. This is not different for aperiodic crystals. Also, for incommensurate modulated phases and quasicrystals, the faces may be indexed with wavevectors occurring in the diffraction pattern, the Fourier module (Janner et al., 1980). As examples, we mention the solution of an old riddle in morphology, the indexing of the faces of calaverite (Dam et al., 1985; Janner \& Dam, 1989), the indexing of satellite faces on $\mathrm{Rb}_{2} \mathrm{ZnBr}_{4}$ and $\mathrm{Rb}_{2} \mathrm{ZnCl}_{4}$ (Janner
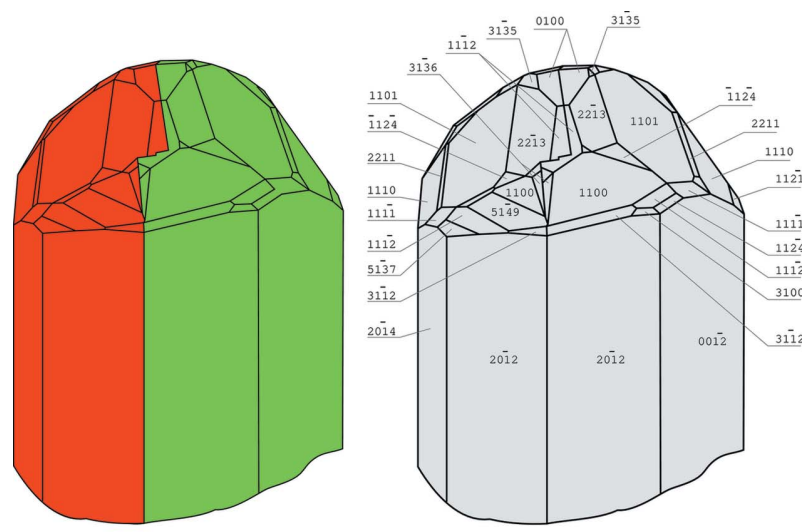

(a)

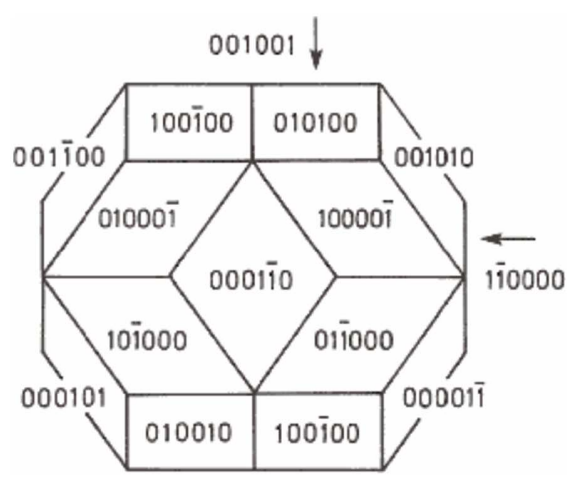

(b)

Figure 47

(a) The faces of a calaverite crystal may be indexed with four indices, in agreement with the aperiodic crystal structure (Dam et al., 1985). (b) The faces of a rhombic triacontahedron with icosahedral symmetry need six indices for their indexing, in agreement with the dimension of the superspace embedding. et al., 1980), and the indexing of the faces of an icosahedral quasicrystal (Fig. 47; Janssen et al., 1989). Other interesting topics that are not covered are the theory of defects in aperiodic crystals (Feuerbacher, 2012) and possible applications (Dubois, 2005).

Another aspect is formed by the scale transformations. The Fourier module of an icosahedral quasicrystal is invariant under multiplication with certain factors, powers of $\tau=(\sqrt{5}-1) / 2$. This has a relation with the normalizer of the point group. This scale invariance occurs in many other places. It may also be used to describe the relation between the various layers of biomolecules. These aspects will be discussed in a forthcoming publication.

\subsection{Summary}

In approximately 50 years the field of aperiodic crystals has developed strongly. After the discovery of the crystallographic nature of incommensurate modulated crystals the field started slowly. In the beginning only a small number of groups was interested. This situation did not change very much with the discovery of incommensurate composites. The big expansion took place after the discovery of quasicrystals. Suddenly, many physicists, crystallographers, chemists, geologists and mathematicians became interested. At the end of the 1970s a series of conferences on this subject started. They were subsequently called 'incommensurate phases', MOSPOQ (modulated phases, polytypes and quasicrystals) and finally Aperiodic. Although the most exciting years are over, there is still a lot of activity. In these years hundreds of scientists have contributed to the state-of-the-art of today.

This does not mean that all problems have been solved. Notwithstanding the progress, there are still many questions, also fundamental ones. The structure determination of modulated phases and composites has developed enormously, but that of quasicrystals was done on an ad hoc basis. The characterization of states (electrons and phonons) in an aperiodic crystal is still not clear. The same holds for the characterization of atomic surfaces in quasicrystals. There are many signs that aperiodic crystals have special properties, but their applications are still limited. Another remaining riddle is the question how a quasicrystal may grow. For conventional lattice periodic crystals, the theory of crystal growth is well developed but it is still a mystery how this works for quasicrystals. Finally, one may notice that electrons and phonons in aperiodic crystals at low energy do not behave too differently from those in lattice periodic crystals. However, at higher energy special properties appear. It can be noted that these properties also occur for complex metallic alloys with huge unit cells. Therefore, one may ask the question whether some of the special properties are really due to the aperiodicity. There is still much work to be done.

We thank all authors who permitted us to reproduce figures from their publications. 


\section{References}

Abe, E., Sato, T. J. \& Tsai, A. P. (1999). Phys. Rev. Lett. 82, 52695272.

Alexander, S. \& McTague, J. (1978). Phys. Rev. Lett. 41, 702-705.

Ao, R., Schaack, G., Schmitt, M. \& Zöller, M. (1989). Phys. Rev. Lett. 62, 183-185.

Bak, P. (1976). Phys. Rev. Lett. 37, 1071-1074.

Bak, P. (1985a). Phys. Rev. Lett. 54, 1517-1519.

Bak, P. (1985b). Phys. Rev. B, 32, 5764- 5772.

Bak, P. (1986). Phys. Rev. Lett. 56, 861-864.

Bak, P. \& Janssen, T. (1978). Phys. Rev. B, 17, 436-439.

Ball, M. D. \& Lloyd, D. J. (1985). Scr. Metall. 19, 1065-1068.

Bendersky, L. (1985). Phys. Rev. Lett. 55, 1461-1463.

Beest, B. van, Janner, A. \& Blinc, R. (1982). J. Phys. C, 16, 5409-5416.

Bieberbach, L. (1911). Math. Ann., LXX, 297-336.

Bieberbach, L. (1912). Math. Ann., LXXII, 401-412.

Bienenstock, A. \& Ewald, P. P. (1962). Acta Cryst. 15, 1253-1261.

Bindi, L., Steinhardt, P. J., Yao, N. \& Lu, P. J. (2009). Science, 324, 1306-1308.

Boissieu, M. de (2012). Chem. Soc. Rev. 41, 6778-6786.

Boissieu, M. de, Francoual, S., Mihalkovič, M., Shibata, K., Baron, A. Q. R., Sidis, Y., Ishimasa, T., Wu, D., Lograsso, T., Regnault, L. P., Gähler, F., Tsutsui, S., Hennion, B., Bastie, P., Sato, T. J., Takakura, H., Currat, R. \& Tsai, A. P. (2007). Nat. Mater. 6, 977984. Boissieu, M. de, Janot, C., Dubois, J. M, Audier, M. \& Dubost, B. (1991). J. Phys. C, 3, 1-25.

Bruijn, N. G. de (1981). Proc. Ser. A, 84, 27-37.

Boudard, M., de Boissieu, M., Janot, C., Heger, G., Beeli, C., Nissen, H.-U., Vincent, H., Ibberson, R., Audier, M. \& Dubois, J.-M. (1992). J. Phys. Condens. Matter, 4, 10149-10168.

Brouns, E., Visser, J. W. \& de Wolff, P. M. (1964). Acta Cryst. 17, 614.

Brown, H., Bülow, R., Neubüser, J., Wondratschek, H. \& Zassenhaus, H. (1978). Crystallographic Groups of Four-Dimensional Space. New York: John Wiley and Sons.

Brown, I. D., Cutforth, B. D., Davies, C. G., Gillespie, R. J., Ireland, P. R. \& Vekris, J. E. (1974). Can. J. Chem. 52, 791-793.

Cahn, J., Gratias, D. \& Mozer, B. (1988). Phys. Rev. B, 38, 1638-1642. Cahn, J. W., Shechtman, D. \& Gratias, D. (1986). J. Mater. Res. 1, 13. Cervellino, A., Haibach, T. \& Steurer, W. (2002). Acta Cryst. B58, 833.

Chapuis, G. (2013). Software Tools for Aperiodic Crystallographers, http://superspace.epfl.ch.

Coddens, G., Bellissent, R., Calvayrac, Y. \& Ambroise, J. P. (1991). Europhys. Lett. 16, 271-276.

Coddens, G., Lyonnard, S. \& Calvarac, Y. (1997). Phys. Rev. Lett. 78, 4209-4212.

Conrad, M., Krumeich, F. \& Harbrecht, B. (1998). Angew. Chem. Int. Ed. 37, 1381-1386.

Cutforth, B. D., Datars, W. R., Gillespie, R. J. \& van Schijndel, A. (1976). Adv. Chem. 150, 56-62.

Dam, B., Janner, A. \& Donnay, J. D. H. (1985). Phys. Rev. Lett. 55, 2301-2304.

Dehlinger, U. (1927). Z. Kristallogr. 65, 615-631.

Dehlinger, U. (1929). Anal. Phys. 5, 749-793.

Dolinsšek, J., Apih, T., Jeglič, P., Feuerbacher, M., Calvo-Dahlborg, M., Dahlborg, U. \& Dubois, J. M. (2002). Phys. Rev. B, 65, 212203.

Dubois, J.-M. (2005). Useful Quasicrystals, 481pp. Singapore: World Scientific.

Dubois, J.-M. \& Janot, C. (1987). J. Phys. 48, 1981-1989.

Dubost, B., Lang, J.-M., Tanaka, M., Saintfort, P. \& Audier, M. (1986). Nature, 324, 48-50.

Duneau, M. \& Katz, A. (1985). Phys. Rev. Lett. 54, 2688-2691.

Elliott, R. J. (1965). Theory of Magnetism in the Rare Earth Metals, Magnetism IIA, edited by G. T. Rado \& H. Schul, pp. 385-424. New York: Academic Press.

Elser, V. (1985). Phys. Rev. B, 32, 4892-4898.

Elser, V. (1986). Acta Cryst. A42, 36-43.
Elser, V. \& Henley, C. L. (1985). Phys. Rev. Lett. 55, 2883-2886.

Enz, U. (1960). Physica, 698-699,

Ephraïm, M., Janssen, T., Janner, A. \& Thiers, A. (2003). International Tables for Crystallography, edited by A. Authier, Vol. D, pp. 62-71. Dordrecht: Kluwer Academic Publishers.

Fast, G. \& Janssen, T. (1968). Technical Report, pp. 6-68. K.U. Nijmegen.

Feuerbacher, M. (2012). Chem. Soc. Rev. 41, 6745-6759.

Frank, F. C. \& van der Merwe, J. H. (1949). Proc. R. Soc. London A, 198, 205-216.

Fujiwara, T. \& Ishii, Y. (2008). Editors. Quasicrystals. Amsterdam: Elsevier.

Gähler, F. \& Rhyner, J. (1986). J. Phys. A, 19, 267-277.

Goldman, A. T., Tai, K., Kreyssig, A., Jesche, A., Ramazanoglu, M., Dennis, K. W., Budko, S. L. \& Canfield, P. C. (2013). Nature Mater. Lett. 3672, 1-5.

Gratias, D., Cahn, J. W. \& Mozer, B. (1988). Phys. Rev. B, 38, $1643-$ 1646.

Gratias, D., Puyraymond, F., Quiquandon, M. \& Katz, A. (2000). Phys. Rev. B, 63, 024202.

Guo, J. Q., Abe, E. \& Tsai, A. P. (2002). Philos. Mag. Lett. 82, $27-$ 35

Guyot, P. \& Audier, M. (1985). Philos. Mag. B, 52, L15-L19.

Haibach, T. \& Steurer, W. (1996). Acta Cryst. A52, 277-286.

Hao, Q., Liu, Y.-w. \& Fan, H.-F. (1987). Acta Cryst. A43, 820-824.

Hayashida, K., Dotera, T., Takano, A. \& Matsushita, Y. (2007). Phys. Rev. Lett. 98, 195502.

Iizumi, M., Axe, J. D., Shirane, G. \& Simaoka, K. (1977). Phys. Rev. B, 15, 4392-4411.

Ishii, Y. \& Fujiwara, T. (2008). Editors. Electronic Structures and Stability Mechanism of Quasicrystals, Ch. 6, pp. 171-208. Amsterdam: Elsevier.

Ishimasa, T., Nissen, H. U. \& Fukano, Y. (1985). Phys. Rev. Lett. 55, 511-513.

Janner, A. (1991). Acta Cryst. A47, 577-590.

Janner, A. (2013). Acta Cryst. A69, 151-163.

Janner, A. \& Dam, B. (1989). Acta Cryst. A45, 115-123.

Janner, A. \& Janssen, T. (1971). Physica, 53, 1-27.

Janner, A. \& Janssen, T. (1977). Phys. Rev. B, 15, 643-658.

Janner, A. \& Janssen, T. (1980). Acta Cryst. A36, 399-408.

Janner, A., Rasing, T., Bennema, P. \& van der Linden, W. H. (1980). Phys. Rev. Lett. 45, 1700-1702.

Janot, C. (1997). Quasicrystals, a Primer, 2nd ed. Oxford University Press.

Janssen, T. (1972). Phys. Kond. Mater. 15, 142-157.

Janssen, T. (1986). Acta Cryst. A42, 261-271.

Janssen, T. (1988). Phys. Rep. 168, 55-113.

Janssen, T. (1991). Acta Cryst. A47, 243-255.

Janssen, T. (1992). Philos. Mag. B, 66, 125-134.

Janssen, T. (2003a). International Tables for Crystallography, edited by A. Authier, Vol. D, 34-62. Dordrecht: Kluwer Academic Publishers.

Janssen, T. (2003b). International Tables for Crystallography, edited by A. Authier, Vol. D, 243-264. Dordrecht: Kluwer Academic Publishers.

Janssen, T. (2014). Symmetry, 6, 171-188.

Janssen, T., Birman, J. L., Dénoyer, F., Koptsik, V. A., Verger-Gaugry, J. L., Weigel, D., Yamamoto, A., Abrahams, S. C. \& Kopsky, V. (2002). Acta Cryst. A58, 605-621.

Janssen, T., Chapuis, G. \& de Boissieu, M. (2007). Aperiodic Crystals. From Modulated Phases to Quasicrystals, 466pp. Oxford University Press.

Janssen, T. \& de Boissieu, M. (2014). C. R. Phys. 15, 58-69.

Janssen, T. \& Janner, A. (1984). Physica A, 126, 163-176.

Janssen, T. \& Janner, A. (1987). Adv. Phys. 36, 519-624.

Janssen, T., Janner, A. \& Ascher, E. (1969). Physica, 42, 41-70.

Janssen, T., Janner, A. \& Bennema, P. (1989). Philos. Mag. B, 59, $233-$ 242. 
Janssen, T., Janner, A., Looijenga-Vos, A. \& de Wolff, P. M. (1992). International Tables for Crystallography, edited by A. J. C. Wilson, Vol. C, pp. 797-836. Dordrecht: Kluwer Academic Publishers.

Johnson, C. K. \& Watson, C. R. (1976). J. Chem. Phys. 64, 2271-2286. Jurdik, E., Myszkiewicz, G., Hohlfeld, J., Tsukamoto, A., Toonen, A. J., van Etteger, A. F., Gerritsen, J., Hermsen, J., GoldbachAschemann, S., Meerts, W. L., van Kempen, H. \& Rasing, Th. (2004). Phys. Rev. B, 69, 201102.

Kalugin, P. A., Kitayev, A. Y. \& Levitov, L. S. (1985a). J. Phys. Lett. 46, L601-607.

Kalugin, P. A., Kitayev, A. Y. \& Levitov, L. S. (1985b). JETP Lett. 41, 145-149.

Katz, A. \& Gratias, D. (1995). Chemical Order and Local Configurations in AlCuFe-type Icosahedral Phase, Proceedings of the 5th International Conference on Quasicrystals, edited by $\mathrm{C}$. Janot \& R. Mosseri, pp. 164-167. Singapore: World Scientific.

Korekawa, M. (1967). Thesis. Ludwig-Maximilian-Universität München, Germany.

Krajči, M. \& Hafner, J. (1997a). Mater. Sci. Eng. A, pp. 226-228.

Krajči, M. \& Hafner, J. (1997b). Mater. Sci. Eng. A, pp. 950-960.

Kramer, P. \& Neri, R. (1984). Acta Cryst. A40, 580-587.

Levine, D. \& Steinhardt, P. (1984). Phys. Rev. Lett. 53, 2477-2480.

Levine, D. \& Steinhardt, P. (1986). Phys. Rev. B, 34, 596-616.

Lifshitz, R. (1998). Phys. Rev. Lett. 80, 2717-2720.

Los, J., Janssen, T. \& Gähler, F. (1993a). J. Non-Cryst. Solids, pp. 153154.

Los, J., Janssen, T. \& Gähler, F. (1993b). J. Non-Cryst. Solids, pp. 581585.

Lubensky, T. C., Ramaswamy, S. \& Toner, J. (1985). Phys. Rev. B, 32 , 7444-7452.

Mackay, A. L. (1982). Physica A, 114, 609-613.

McGrath, R., Smerdon, J. A., Sharma, H. R., Theis, W. \& Ledieu, J. (2010). J. Phys. Condens. Matter, 22, 084022.

McMahon, M. I. \& Nelmes, R. J. (2006). Chem. Soc. Rev. 35, 943-963.

Mermin, N. D. (1992). Rev. Mod. Phys. 64, 3-49.

Mihalkovič, M., Henley, C., Widom, M. \& Ganesh, P. (2008). ArXiv: 0802.2926v2.

Mizutani, U., Inukai, M., Sato, H. \& Zijlstra, E. S. (2012). Chem. Soc. Rev. 41, 67996820.

Oszlányi, G. \& Sütô, A. (2004). Acta Cryst. A60, 134-141.

Overhauser, A. W. (1971). Phys. Rev. B, 3, 3173-3182.

Palatinus, L. (2013). Acta Cryst. B69, 1-16.

Penrose, R. (1974). Bull. Inst. Math. Appl. 10, 266-271.

Penrose, R. (1979). Math. Intell. 2, 32-37.

Perez-Mato, J. M., Madariaga, G. \& Tello, M. J. (1984). Phys. Rev. B, 30, 1534-1543.

Perez-Mato, J. M., Ribeiro, J. L., Petricek, V. \& Aroyo, M. I. (2012). J. Phys. Condens. Matter, 24, 163201.

Petřiček, V., Dušek, M. \& Palatinus, L. (2006). JANA2006. Institute of Physics, Praha, Czech Republic.

Pikin, S. A. (1986). Incommensurate Phases in Dielectrics, edited by R. Blinc \& A. P. Levanyuk, pp. 319-366. Amsterdam: Elsevier.

Pouget, J. P., Shirane, G., Hasting, J. M., Heeger, A. J., Miro, N. D. \& MacDiarmid, A. G. (1978). Phys. Rev. B, 18, 3645-3656.

Quilichini, M. \& Janssen, T. (1997). Rev. Mod. Phys. 69, 277-314.

Quiquandon, M. \& Gratias, D. (2006). Phys. Rev. B, 74, 214005.

Rabson, D. A., Mermin, N. D., Rokhsar, D. S. \& Wright, D. C. (1991). Rev. Mod. Phys. 63, 699-733.

Rokhsar, D. S., Wright, D. C. \& Mermin, N. D. (1988). Acta Cryst. A44, 197-211.

Schobinger-Papamantellos, P. \& Janssen, T. (2006). Z. Kristallogr. 221, 732-734.

Schobinger-Papamantellos, P., Rodrguez-Carvajal, J., Andàe, G. \& Buschow, K. H. J. (2006). J. Magn. Magn. Mater. 300, 333-350.

Schobinger-Papamantellos, P., Rodrguez-Carvajal, J., Ritter, C. \& Buschow, K. H. J. (2010). J. Magn. Magn. Mater. 322, 119-132.

Selke, W. (1988). Phys. Rep. 170, 213-264.

Selke, W. \& Duxbury, P. M. (1984). Z. Phys. B, 57, 49-58.
Sharma, H. R., Fourñee, V., Shimoda, M., Ross, A. R., Lograsso, T., Tsai, A. P. \& Yamamoto, A. (2004). Phys. Rev. Lett. 93, 165502.

Shechtman, D., Blech, I., Gratias, D. \& Cahn, J. W. (1984). Phys. Rev. Lett. 20, 1951-1953.

Souvignier, B. (2006). Z. Kristallogr. 221, 77-82.

Special Interest Group on Aperiodic Crystals (1998). Software for aperiodic crystals, http://sig3.ecanews.org.

Sterzel, R., Gross, C., Kounis, A., Miehe, G., Fuess, H., Reutzel, S., Holland-Moritz, D. \& Assmus, W. (2002). Philos. Mag. Lett. 82, 443-450.

Steurer, W., Haibach, T., Zhang, B., Kek, S. \& Lück, R. (1993). Acta Cryst. B49, 661-675.

Steurer, W. \& Deloudi, S. (2009). Crystallography of Quasicrystals, Springer Series in Materials Science, 384pp. New York: Springer.

Stokes, H. T., Campbell, B. J. \& Cordes, R. (2013). Acta Cryst. A69, 388-395.

Stokes, H. T., Campbell, B. J. \& van Smaalen, S. (2011). Acta Cryst. A67, 45-55.

Takakura, H., Yamamoto, A. \& Tsai, A. P. (2001). Acta Cryst. A57, 576-585.

Takakura, H., Pay Gomez, C., Yamamoto, A., de Boissieu, M. \& Tsai, A. P. (2007). Nat. Mater. 6, 58-63.

Talapin, D. V., Shevchenko, E. V., Bodnarchuk, M. I., Ye, X., Chen, J. \& Murray, C. B. (2009). Nature, 461, 964-967.

Taniguchi, S. \& Abe, E. (2008). Philos. Mag. B, 88, 1949-1958.

Toudic, B., Garcia, P., Odin, C., Rabiller, Ph., Ecolivet, C., Cllet, E., Bourges, P., MacIntyre, G. J., Hollingsworth, M. D. \& Breczewski, T. (2008). Science, 319, 69-71.

Toudic, B., Rabiller, P., Bourgeois, L., Huard, M., Ecolivet, C., McIntyre, G. J., Bourges, P., Breczewski, T. \& Janssen, T. (2011). Eur. Phys. Lett. 93, 16003.

Trambly de Laissardière, G. \& Mayou, D. (2014). C. R. Phys. 50, 7081.

Tsai, A. P. (2013). Chem. Soc. Rev. 42, 5352-5365.

Tsai, A. P., Guo, J. Q., Abe, E., Takakura, H. \& Sato, T. J. (2000). Nature, 408, 537-538.

Tsai, A. P., Inoue, A. \& Masumoto, T. (1987). J. Mater. Sci. Lett. 6, 1403-1405.

Tsai, A. P., Inoue, A. \& Matsumoto, T. (1989). Mater. Trans. JIM, 30, 463-473.

Tsai, A. P., Inoue, A., Yokoyama, Y. \& Masumoto, T. (1990). Mater. Trans. JIM, 31, 98-103.

Tsai, A. P., Niikura, A., Inoue, A., Masumoto, T., Nishida, Y., Tsuda, K. \& Tanaka, M. (1994). Philos. Mag. Lett. 70, 169-175.

Ungar, G. \& Zeng, X. (2005). Soft Matter, 1, 95-106.

van Smaalen, S. (1991). Phys. Rev. B, 43, 11330-11341.

van Smaalen, S. (2007). Incommensurate Crystallography, $270 \mathrm{pp}$. Oxford University Press.

van Smaalen, S., Palatinus, L. \& Schneider, M. (2003). Acta Cryst. A59, 459-469.

Verbin, M., Zilberberg, O., Kraus, Y. E., Lahini, Y. \& Silberberg, Y. (2013). Phys. Rev. Lett. 110, 076403.

Wang, N., Chen, H. \& Kuo, K. H. (1987). Phys. Rev. Lett. 59, 10101013.

Weber, S. \& Yamamoto, A. (1997). Philos. Mag. A, 76, 85-106.

Wolff, P. M. de (1974). Acta Cryst. A30, 777-785.

Wolff, P. M. de (1977). Acta Cryst. A33, 493-497.

Wolff, P. M. de, Janssen, T. \& Janner, A. (1981). Acta Cryst. A37, 625636.

Wolff, P. M. de \& van Aalst, W. (1972). Acta Cryst. A28, S111.

Yamamoto, A. (1980). Phys. Rev. B, 22, 373-379.

Yamamoto, A. (1996). Acta Cryst. A52, 509-560.

Yamamoto, A. \& Hiraga, K. (1988). Phys. Rev. B, 37, 6207-6214.

Yamamoto, A. (2011). http://wcp-ap.eng.hokudai.ac.jp/yamamoto/.

Yamamoto, A., Janssen, T., Janner, A. \& de Wolff, P. M. (1985). Acta Cryst. A41, 528-530.

Yamamoto, A., Takakura, H. \& Tsai, A. P. (2003). Phys. Rev. B, 68, 094201. 
Zassenhaus, H. (1948). Commun. Helv. Math. 21, 117- Zijlstra, E. S., Bose, S. K., Klanšek, M., Jeglitč, P. \& Dolinšek, J. 141. (2005). Phys. Rev. B, 72, 174206.

Zeng, X., Ungar, G., Liu, Y., Percec, V., Dulcey, A. E. \& Hobbs, J. K. $\quad$ Zijlstra, E. S., Fasolino, A. \& Janssen, T. (1999). Phys. Rev. B, 59, 302(2004). Nature, 428, 157-160. 307. 